\title{
A Concise Synthetic Method for 1,3,5-Triazinane-2,4-Dithiones
}

\author{
Zheng Li,*Hongfang Cai, Jingya Yang, Pengxian Niu and Chenhui Liu \\ College of Chemistry and Chemical Engineering, Northwest Normal University, Lanzhou, Gansu, \\ 730070, P. R. China
}

\begin{abstract}
1,3,5-Triazinane-2,4-ditionas foram sintetizadas eficientemente via condensação de 2 equiv. de 1-aril-tiouréa com 1 equiv. de ácidos carboxílicos alifáticos usando cloreto férrico hexahidratado como catalisador. Este protocolo tem as vantagens de alto rendimento, condições brandas e procedimento simples.
\end{abstract}

1,3,5-Triazinane-2,4-dithiones were efficiently synthesized via condensation of 2 equiv. of 1 -arylthioureas with 1 equiv. of aliphatic carboxylic acids using ferric chloride hexahydrate as a catalyst. This protocol has the advantages of high yield, mild condition and simple procedure.

Keywords: 1,3,5-triazinane-2,4-dithione, 1,3,5-triazinane, thiourea, carboxylic acid, synthesis

\section{Introduction}

Heterocyclic compounds have long been known to exhibit remarkable biological and pharmacological properties. ${ }^{1}$ Among the heterocycles, triazine derivatives have attracted much attention because they can connect with other molecules by hydrogen or coordination bond to form network supramolecular materials, ${ }^{2}$ and they can serve as luminescent or n-type electron-carrying materials after connected with some substituents like diphenylacetylene, naphthalene and anthracene. ${ }^{3}$ Triazine derivatives constitute well-known compounds that have been used as fungicidal, ${ }^{4}$ antiplasmodial, ${ }^{5}$ anti-HIV ${ }^{6}$ and herbicidal agents, ${ }^{7}$ and chiral discriminators, ${ }^{8}$ hydrogen sulfide scavengers ${ }^{9}$ and low-toxicity drug deliverers. ${ }^{10}$ They were also applied in organic synthesis, ${ }^{11}$ enantiodifferentiating coupling reagents, ${ }^{12}$ catalysis, ${ }^{13}$ molecular tectonics, ${ }^{14}$ and polymeric materials. ${ }^{15}$ Meanwhile, heterocycles containing a thiourea structural unit have a special place among pharmaceutically important natural and synthetic materials, showing powerful antiproliferative action, ${ }^{16}$ antibacterial properties ${ }^{17}$ and anticancer activity. ${ }^{18}$ For these reasons, 1,3,5-triazinane derivatives incorporating thiourea unit may be important in many fields.

The general synthetic methods for 1,3,5-triazinane derivatives involve the reactions of $N, N^{\prime}$-bis(arylmethylidene)arylmethane diimines with thioureas, ${ }^{19}$ the

*e-mail: lizheng@nwnu.edu.cn multi-component reactions of phosphonates, nitriles, aldehydes and isocyanates ${ }^{20}$ the condensation of trifluoromethanesulfonamide with formaldehyde, ${ }^{21}$ and the reactions of thiosemicarbazones with potassium thiocyanate and benzoyl chloride. ${ }^{22}$ However, some methods use expensive reagents, toxic organic solvents, rigorous conditions, tedious workup procedure and long reaction time. Therefore, it is necessary to develop simple and efficient synthetic methods to 1,3,5-triazinane derivatives.

In this article, we report the synthesis of 1,3,5-triazinane2,4-dithiones by reactions of 2 equiv. of 1-arylthioureas with 1 equiv. of aliphatic carboxylic acids using ferric chloride hexahydrate as a catalyst.

\section{Results and Discussion}

Initially, the synthesis of 1,3,5-triazinane-2,4-dithione was attempted by reaction of 1-phenylthiourea with acetic acid at room temperature under catalyst-free condition, however, no product was observed. Subsequently, the mixture of 1-phenylthiourea and acetic acid was heated at $80{ }^{\circ} \mathrm{C}$ for several hours, a new compound was isolated in low yield, which was identified to be a novel heterocyclic compound, 6-hydroxy-6-methyl-1,5-diphenyl1,3,5-triazinane-2,4-dithione. In our further research, it was found that some Brønsted acids, such as $p$-toluenesulfonic acid (PTSA) and trichloroacetic acid (TCA), and Lewis acids, such as $\mathrm{AlCl}_{3}, \mathrm{CuCl}_{2}, \mathrm{NiCl}_{2}, \mathrm{FeCl}_{3}$ and $\mathrm{FeCl}_{3} \cdot 6 \mathrm{H}_{2} \mathrm{O}$, 
could efficiently catalyze the reaction (Table 1 ). Among them, $\mathrm{FeCl}_{3} \cdot 6 \mathrm{H}_{2} \mathrm{O}$ could give the best yield if the reaction was carried out at $80^{\circ} \mathrm{C}$ using $10 \mathrm{~mol} \%$ amount of catalyst (Table 1, entry 8). In addition, in this reaction, acetic acid was acted as a reactant and solvent.

To explore the generality and scope of the synthetic reactions, and synthesis of a series of 6-hydroxy-6-alkyl1,5-diaryl-1,3,5-triazinane-2,4-dithiones (Scheme 1), different 1-arylthioureas and aliphatic carboxylic acids as substrates were examined under optimal conditions (Table 2). It was found that various 1-arylthioureas could efficiently react with aliphatic carboxylic acids at $80{ }^{\circ} \mathrm{C}$ in the presence of $\mathrm{FeCl}_{3} \cdot 6 \mathrm{H}_{2} \mathrm{O}$ to give the corresponding products in high yield. In comparison with 1-phenylthiourea, it was found that 1-arylthioureas including electron-donating groups, such as methyl and methoxyl, on the aromatic rings gave the corresponding products in higher yield and in faster rate (Table 2, entries 7-18). 1-Arylthioureas bearing electron-withdrawing substituents, such as chloro, on aromatic rings gave the corresponding product in slightly lower yield under similar conditions (Table 1, entry 19). Aliphatic carboxylic acids from $\mathrm{C}_{2}-\mathrm{C}_{7}$ were examined for the reactions, and afforded the corresponding products in high yield. In addition, aromatic carboxylic acids, such as various (un)substituted benzoic acids, were also attempted for the similar reactions, but no desired products were observed.

The resulting compounds 6-hydroxy-6-alkyl-1,5-diaryl1,3,5-triazinane-2,4-dithiones are highly soluble in polar organic solvents including $\mathrm{CHCl}_{3}, \mathrm{CH}_{2} \mathrm{Cl}_{2}, \mathrm{DMSO}$, $\mathrm{DMF}$ and $\mathrm{EtOH}$, but insoluble in toluene, benzene, ether and $n$-hexane. The structures of all compounds were identified by infrared (IR), ${ }^{1} \mathrm{H}$ and ${ }^{13} \mathrm{C}$ nuclear magnetic resonance (NMR) spectroscopies and elemental analysis. The ${ }^{1} \mathrm{H}$ NMR spectra of 6-hydroxy-6-alkyl-1,5-diaryl1,3,5-triazinane-2,4-dithiones show the singlets of hydroxyls at 5.89-6.12 ppm and the multiplets of aromatic rings at $6.84-8.12 \mathrm{ppm}$. The IR spectra show the characteristic adsorption of hydroxyls at $3359-3450 \mathrm{~cm}^{-1}$.

A possible mechanism for the synthesis of 1,3,5-triazinane-2,4-dithione is shown in Scheme 2.

Table 1. Synthesis of 6-hydroxy-6-methyl-1,5-diphenyl-1,3,5-triazinane-2,4-dithione under different conditions ${ }^{\mathrm{a}}$

2

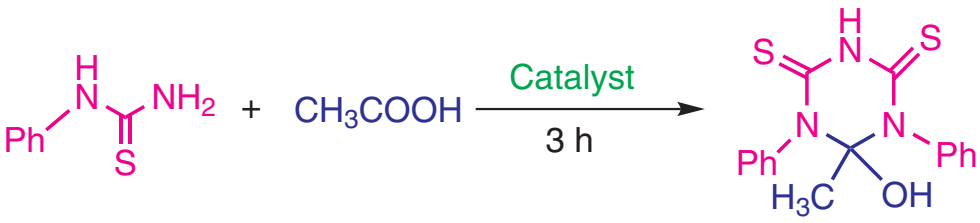

\begin{tabular}{|c|c|c|c|c|}
\hline entry & Catalyst & Amount of catalyst / $(\mathrm{mol} \%)$ & Temperature $/{ }^{\circ} \mathrm{C}$ & Yield $/ \%^{\mathrm{b}}$ \\
\hline 1 & - & 0 & 80 & 10 \\
\hline 2 & PTSA & 10 & 80 & 48 \\
\hline 3 & TCA & 10 & 80 & 56 \\
\hline 4 & $\mathrm{AlCl}_{3}$ & 10 & 80 & 51 \\
\hline 5 & $\mathrm{CuCl}_{2}$ & 10 & 80 & 60 \\
\hline 6 & $\mathrm{NiCl}_{2}$ & 10 & 80 & 70 \\
\hline 7 & $\mathrm{FeCl}_{3}$ & 10 & 80 & 74 \\
\hline 8 & $\mathrm{FeCl}_{3} \cdot 6 \mathrm{H}_{2} \mathrm{O}$ & 10 & 80 & 88 \\
\hline 9 & $\mathrm{FeCl}_{3} \cdot 6 \mathrm{H}_{2} \mathrm{O}$ & 10 & 20 & 20 \\
\hline 10 & $\mathrm{FeCl}_{3} \cdot 6 \mathrm{H}_{2} \mathrm{O}$ & 10 & 40 & 46 \\
\hline 11 & $\mathrm{FeCl}_{3} \cdot 6 \mathrm{H}_{2} \mathrm{O}$ & 10 & 60 & 70 \\
\hline 12 & $\mathrm{FeCl}_{3} \cdot 6 \mathrm{H}_{2} \mathrm{O}$ & 10 & 100 & 60 \\
\hline 13 & $\mathrm{FeCl}_{3} \cdot 6 \mathrm{H}_{2} \mathrm{O}$ & 5 & 80 & 85 \\
\hline 14 & $\mathrm{FeCl}_{3} \cdot 6 \mathrm{H}_{2} \mathrm{O}$ & 15 & 80 & 86 \\
\hline
\end{tabular}

${ }^{a}$ Reaction conditions: 1-phenylthiourea $(2 \mathrm{mmol})$, acetic acid ( $\left.3 \mathrm{mmol}\right)$ under different conditions; bisolated yields.

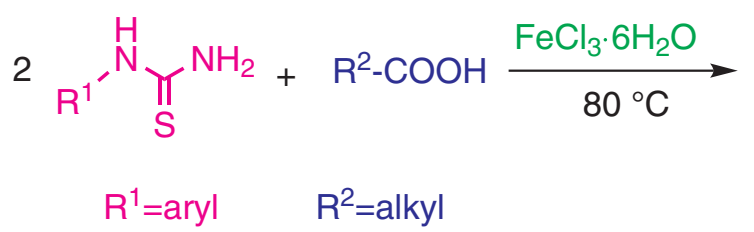<smiles>[R7]N1C(=S)NC(=S)N([R7])C1([R])O</smiles>

Scheme 1. Synthesis of 6-hydroxy-6-alkyl-1,5-diaryl-1,3,5-triazinane-2,4-dithiones. 
Table 2 Synthesis of 6-hydroxy-6-alkyl-1,5-diaryl-1,3,5-triazinane-2,4-dithiones ${ }^{\text {a }}$

\begin{tabular}{|c|c|c|c|c|c|}
\hline entry & $\mathrm{R}^{1}$ & $\mathrm{R}^{2}$ & time $/ \mathrm{h}$ & $\mathrm{mp} /{ }^{\circ} \mathrm{C}$ & Yield / \% \\
\hline 1 & $\mathrm{C}_{6} \mathrm{H}_{5}$ & $\mathrm{CH}_{3}$ & 3 & $254-256$ & 87 \\
\hline 2 & $\mathrm{C}_{6} \mathrm{H}_{5}$ & $\mathrm{C}_{2} \mathrm{H}_{5}$ & 3 & $197-198$ & 85 \\
\hline 3 & $\mathrm{C}_{6} \mathrm{H}_{5}$ & $n-\mathrm{C}_{3} \mathrm{H}_{7}$ & 3 & $133-135$ & 84 \\
\hline 4 & $\mathrm{C}_{6} \mathrm{H}_{5}$ & $n-\mathrm{C}_{4} \mathrm{H}_{9}$ & 4 & $155-157$ & 80 \\
\hline 5 & $\mathrm{C}_{6} \mathrm{H}_{5}$ & $n-\mathrm{C}_{5} \mathrm{H}_{11}$ & 4 & $124-126$ & 80 \\
\hline 6 & $\mathrm{C}_{6} \mathrm{H}_{5}$ & $n-\mathrm{C}_{6} \mathrm{H}_{13}$ & 4 & 105-106 & 79 \\
\hline 7 & $2-\mathrm{CH}_{3} \mathrm{C}_{6} \mathrm{H}_{4}$ & $\mathrm{CH}_{3}$ & 3 & $239-241$ & 82 \\
\hline 8 & $2-\mathrm{CH}_{3} \mathrm{C}_{6} \mathrm{H}_{4}$ & $\mathrm{C}_{2} \mathrm{H}_{5}$ & 3 & $160-162$ & 88 \\
\hline 9 & $2-\mathrm{CH}_{3} \mathrm{C}_{6} \mathrm{H}_{4}$ & $n-\mathrm{C}_{3} \mathrm{H}_{7}$ & 3 & $154-155$ & 83 \\
\hline 10 & $2-\mathrm{CH}_{3} \mathrm{C}_{6} \mathrm{H}_{4}$ & $n-\mathrm{C}_{4} \mathrm{H}_{9}$ & 4 & $123-124$ & 85 \\
\hline 11 & 4- $\mathrm{CH}_{3} \mathrm{C}_{6} \mathrm{H}_{4}$ & $\mathrm{C}_{2} \mathrm{H}_{5}$ & 2 & $119-121$ & 88 \\
\hline 12 & 4- $\mathrm{CH}_{3} \mathrm{C}_{6} \mathrm{H}_{4}$ & $n-\mathrm{C}_{3} \mathrm{H}_{7}$ & 2 & $127-129$ & 90 \\
\hline 13 & 4- $\mathrm{CH}_{3} \mathrm{C}_{6} \mathrm{H}_{4}$ & $n-\mathrm{C}_{4} \mathrm{H}_{9}$ & 2 & $126-127$ & 86 \\
\hline 14 & 4- $\mathrm{CH}_{3} \mathrm{C}_{6} \mathrm{H}_{4}$ & $n-\mathrm{C}_{5} \mathrm{H}_{11}$ & 3 & $111-113$ & 80 \\
\hline 15 & 4- $\mathrm{CH}_{3} \mathrm{C}_{6} \mathrm{H}_{4}$ & $n-\mathrm{C}_{6} \mathrm{H}_{13}$ & 3 & 103-104 & 81 \\
\hline 16 & $4-\mathrm{CH}_{3} \mathrm{OC}_{6} \mathrm{H}_{4}$ & $\mathrm{CH}_{3}$ & 3 & 218-219 & 80 \\
\hline 17 & $4-\mathrm{CH}_{3} \mathrm{OC}_{6} \mathrm{H}_{4}$ & $\mathrm{C}_{2} \mathrm{H}_{5}$ & 4 & $243-245$ & 84 \\
\hline 18 & 4- $\mathrm{CH}_{3} \mathrm{OC}_{6} \mathrm{H}_{4}$ & $n-\mathrm{C}_{3} \mathrm{H}_{7}$ & 4 & $219-221$ & 78 \\
\hline 19 & 4- $\mathrm{ClC}_{6} \mathrm{H}_{4}$ & $\mathrm{CH}_{3}$ & 4 & 208-210 & 66 \\
\hline
\end{tabular}

aReaction conditions: 1-arylthiourea $(2 \mathrm{mmol})$, aliphatic carboxylic acid $(3 \mathrm{mmol})$ and ferric chloride hexahydrate $(0.2 \mathrm{mmol})$ at $80{ }^{\circ} \mathrm{C}$; bisolated yields.<smiles>[R7]NC(N)=S</smiles><smiles>[R7]NC(=S)NC(=S)N[R7]</smiles>

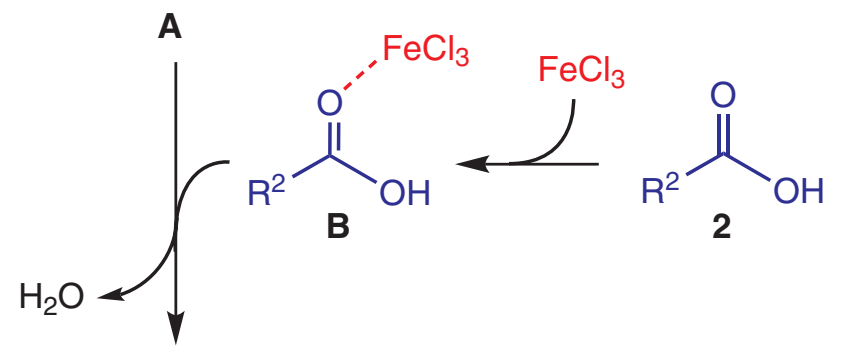<smiles>[R7]N1C(=S)NC(=S)N([R7])C1([R])O</smiles><smiles>[R]C(=O)N([R])C(=S)NC(=S)N[R]Cl</smiles>

Scheme 2. The possible mechanism for the synthesis of 1,3,5-triazinane-2,4-dithiones.

Presumably, condensation of 2 equiv. of 1-arylthiourea 1 releasing a mole of ammonia first generates an intermediate A. One of amino groups of $\mathbf{A}$ subsequent reacts with a mole of complex $\mathbf{B}$, which is formed from aliphatic carboxylic acid $\mathbf{2}$ and ferric chloride in the solution, to give intermediate $\mathbf{C}$ by loss of water. Subsequently the carbonyl 
group of $\mathbf{C}$ undergoes the nuleophilic addition of its amino group by releasing ferric chloride to form a six-membered heterocyclic compound, 1,3,5-triazinane-2,4-dithione 3.

\section{Conclusion}

An efficient and concise method has been developed for the synthesis of 1,3,5-triazinane-2,4-dithiones via condensation of 2 equiv. of 1 -arylthioureas with 1 equiv. of aliphatic carboxylic acids using ferric chloride hexahydrate as a catalyst. This protocol has the advantages of high yield, mild condition and simple procedure.

\section{Experimental}

IR spectra were recorded using $\mathrm{KBr}$ pellets on an Alpha Centauri FTIR spectrophotometer and ${ }^{1} \mathrm{H}$ and ${ }^{13} \mathrm{C}$ NMR spectra on a Mercury-400BB instrument using $\mathrm{CDCl}_{3}$ or DMSO- $d_{6}$ as solvents and $\mathrm{Me}_{4} \mathrm{Si}$ as internal standard. Melting points (mp) were observed in an electrothermal melting point apparatus. Flash column chromatography was carried out using 200-300 mesh silica gel at increased pressure. Aromatic thioureas were synthesized according to the literature methods. ${ }^{23}$

General procedure for the preparation of 6-hydroxy6-alkyl-1,5-diaryl-1,3,5-triazinane-2,4-dithiones

The mixture of 1-arylthioureas ( $2 \mathrm{mmol})$, aliphatic carboxylic acids ( $3 \mathrm{mmol}$ ) and ferric chloride hexahydrate $(0.2 \mathrm{mmol})$ was heated at $80{ }^{\circ} \mathrm{C}$ for appropriate time according to Table 2. The progress of the reactions was monitored by TLC (thin layer chromatography). After the completion of the reactions, the systems were cooled to room temperature, and the mixture was subjected to silica gel flash column chromatography (ethyl acetate, petroleum ether, 1:6) to obtain pure products.

\section{Supplementary Information}

Full set of characterization data (IR, ${ }^{1} \mathrm{H}$ and ${ }^{13} \mathrm{C}$ NMR spectra) are available free of charge at http://jbcs.sbq.org.br as PDF file.

\section{Acknowledgements}

The authors thank the National Natural Science Foundation of China (20772096) and Key Laboratory of Polymer Materials of Gansu Province for the financial support of this work.

\section{References}

1. Menicagli, R.; Samaritani, S.; Signore, G.; Vaglini, F.; Via, L. D.; J. Med. Chem. 2004, 47, 4649; Franzen, R. G.; J. Comb. Chem. 2000, 2, 195; Nefzi, A.; Ostresh, J. M.; Houghten, R. A.; Chem. Rev. 1997, 97, 449.

2. Cai, Y. Q.; Wu, W.; Wang, H.; Miyake, J.; Qian, D. J.; Surf. Sci. 2011, 605, 321.

3. Kostas, I. D.; Andreadaki, F. J.; Medlycott, E. A.; Hanan, G. S.; Monflier, E.; Tetrahedron Lett. 2009, 50, 1851; Lee, C. H.; Yamamoto, T.; Tetrahedron Lett. 2001, 42, 3993.

4. Ibrahim, M. A.; Abdel-Rahman, R. M.; Abdel-Halim, A. M.; Ibrahim, S. S.; Allimony, H. A.; J. Braz. Chem. Soc. 2009, 20, 1275.

5. Klenke, B.; Barrett, M. P.; Brun, R.; Gilbert, I. H.; J. Antimicrob. Chemother. 2003, 52, 290.

6. Patel, R. B.; Chikhalia, K. H.; Pannecouque, C.; Clercq, E.; J. Braz. Chem. Soc. 2007, 18, 312.

7. Soong, C. L.; Ogawa, J.; Sakuradani, E.; Shimizu, S.; J. Biol. Chem. 2002, 227, 7051.

8. Sugimoto, H.; Yamane, Y.; Inoue, S.; Tetrahedron: Asymmetry 2000, 11, 2067.

9. Bakke, J. M.; Buhaug, J. B.; Ind. Eng. Chem. Res. 2004, 43, 1962.

10. Murray, A. P.; Miller, M. J.; J. Org. Chem. 2003, 68, 191; Ghosh, M.; Miller, M. J.; J. Org. Chem. 1994, 59, 1020.

11. Falorni, M.; Porcheddu, A.; Taddei, M.; Tetrahedron Lett. 1999, 40, 4395; Luca, L. D.; Giacomelli, G.; Taddei, M.; J. Org. Chem. 2001, 66, 2534; Babak, K.; Hazarkhani, H.; Synthesis 2003, 2547; Dang, Q.; Gomez-Galeno, J. E.; J. Org. Chem. 2002, 67, 8703.

12. Kaminski, Z. J.; Kolesinska, B.; Kaminska, J. E.; Gora, J.; J. Org. Chem. 2001, 66, 6276.

13. Omotowa, B. A.; Shreeve, J. M.; Organometallics 2004, 23, 783; Graeme, C.; Cremiers, H. A.; Rotello, V. M.; Tarbitc, B.; Vanderstraetena, P. E.; Tetrahedron 2001, 57, 2787.

14. Fournier, J. H.; Maris, T.; Wuest, J. D.; J. Org. Chem. 2004, 69, 1762; Laliberte, D.; Maris, T.; Wuest, J. D.; J. Org. Chem. 2004, 69, 1776.

15. Brunsveld, L.; Vekemans, J. A. J. M.; Hirschberg, J. H. K. K.; Sijbesma, R. P.; Meijer, E. W.; Proc. Natl. Acad. Sci. U.S.A. 2002, 99, 4977.

16. Figueiredo, I. M.; Santos, L. V.; Costa, W. F.; Carvalho, J. E.; Silva, C. C.; Sacoman, J. L.; Kohn, L. K.; Sarragiotto, M. H.; J. Braz. Chem. Soc. 2006, 17, 954.

17. Han, T.; Cho, J. H.; Oh, C. H.; Eur. J. Med. Chem. 2006, 41, 825.

18. Yan, K.; Lok, C. N.; Bierla, K.; Che, C. M.; Chem. Commun. 2010, 46, 7691.

19. Kaboudin, B.; Ghasemi, T.; Yokomatsu, T.; Synthesis 2009 , 3089. 
20. Groenendaal, B.; Vugts, D. J.; Schmitz, R. F.; Kanter, F. J. J.; Ruijter, E.; Groen, M. B.; Orru, R. V. A.; J. Org. Chem. 2008, $73,719$.

21. Meshcheryakov, V. I.; Albanov, A. I.; Shainyan, B. A.; Russ. J. Org. Chem. (Engl. Transl.) 2005, 41, 1381.

22. Ali, T. E. S.; Abdel-Monem, W. R.; Phosphorus, Sulfur, Silicon Relat. Elem. 2008, 183, 2161.
23. Thanigaimalai, P.; Hoang, T. A. L.; Lee, K. C.; Bang, S. C.; Sharma, V. K.; Yun, C. Y.; Roh, E.; Hwang, B. Y.; Kim, Y.; Jung, S. H.; Bioorg. Med. Chem. Lett. 2010, 20, 2991; Ubarhande, S. S.; Thakare, V. G.; Berad, B. N.; J. Indian Chem. Soc. 2010, 87, 1137.

Submitted: May 13, 2011 Published online: August 11, 2011 


\title{
A Concise Synthetic Method for 1,3,5-Triazinane-2,4-Dithiones
}

\author{
Zheng Li,* Hongfang Cai, Jingya Yang, Pengxian Niu and Chenhui Liu \\ College of Chemistry and Chemical Engineering, Northwest Normal University, Lanzhou, Gansu, \\ 730070, P. R. China
}

General procedure for the preparation of 6-hydroxy6-alkyl-1,5-diaryl-1,3,5-triazinane-2,4-dithiones

The mixture of 1-arylthioureas ( $2 \mathrm{mmol})$, aliphatic carboxylic acids ( $3 \mathrm{mmol}$ ) and ferric chloride hexahydrate (0.2 mmol) was heated at $80{ }^{\circ} \mathrm{C}$ for appropriate time according to Table 2 . The progress of the reactions was monitored by TLC (thin layer chromatography). After the completion of the reactions, the systems were cooled to room temperature, and the mixture was subjected to silica gel flash column chromatography (ethyl acetate, petroleum ether, 1:6) to obtain pure products. The spectral data and scanned spectra for products are given below.

6-Hydroxy-6-methyl-1,5-diphenyl-1,3,5-triazinane2,4-dithione (1)

White solid, mp $254-256{ }^{\circ} \mathrm{C}$; IR (KBr) $v_{\text {max }} / \mathrm{cm}^{-1} 3409$, 2925, 2861, 1614, 1582, 1533, 1473, 1442, 1301, 1251, 1157, 759, 697; ${ }^{1} \mathrm{H} \mathrm{NMR}\left(\mathrm{CDC1}_{3}, 400 \mathrm{MHz}\right) \delta$ 7.06-7.71 (m, 10H, Ph-H), 6.10 (s, 1H, OH), $2.24\left(\mathrm{~s}, 3 \mathrm{H}, \mathrm{CH}_{3}\right)$; ${ }^{13} \mathrm{C}$ NMR (DMSO- $\left.d_{6}, 100 \mathrm{MHz}\right) \delta 181.6,177.4,147.8$, 139.3, 134.2, 130.0, 129.9 (2C), 128.7 (2C), 128.4 (2C), 122.7, 120.0 (2C), 25.9; Anal. calcd. for $\mathrm{C}_{16} \mathrm{H}_{15} \mathrm{~N}_{3} \mathrm{OS}_{2}$ : C, 58.33; H, 4.59; N, 12.76; Found: C, 58.22; H, 4.57; N, 12.73.

6-Hydroxy-6-ethyl-1,5-diphenyl-1,3,5-triazinane2,4-dithione (2)

White solid, mp $197-198{ }^{\circ} \mathrm{C}$; IR (KBr) $v_{\max } / \mathrm{cm}^{-1} 3359$, 3064, 2986, 2915, 1616, 1579, 1539, 1483, 1443, 1350, 1216 , 1060, 750, 690; ${ }^{1} \mathrm{H} \mathrm{NMR}\left(\mathrm{CDCl}_{3}, 400 \mathrm{MHz}\right) \delta 7.05-7.71(\mathrm{~m}$, $10 \mathrm{H}, \mathrm{Ph}-\mathrm{H}), 6.11$ (s, 1H, OH), 2.51 (q, 2H, J $7.6 \mathrm{~Hz}, \mathrm{CH}_{2}$ ), $1.13\left(\mathrm{t}, 3 \mathrm{H}, J 7.6 \mathrm{~Hz}, \mathrm{CH}_{3}\right) ;{ }^{13} \mathrm{C} \mathrm{NMR}\left(\mathrm{CDCl}_{3}, 100 \mathrm{MHz}\right)$ $\delta 186.7,177.3,145.7,137.8,133.8,130.8(3 \mathrm{C}), 129.2(2 \mathrm{C})$, 128.2 (2C), 123.7, 118.8 (2C), 32.30, 9.50; Anal. calcd. for $\mathrm{C}_{17} \mathrm{H}_{17} \mathrm{~N}_{3} \mathrm{OS}_{2}$ : C, 59.45; H, 4.99; N, 12.23; Found: C, 59.54; H, 4.98; N, 12.19 .

*e-mail: lizheng@nwnu.edu.cn
6-Hydroxy-6-propyl-1,5-diphenyl-1,3,5-triazinane2,4-dithione (3)

White solid, mp $133-135^{\circ} \mathrm{C}$; IR (KBr) $v_{\max } / \mathrm{cm}^{-1} 3410$, 3054, 2960, 2927, 2862, 1601, 1586, 1543, 1475, 1442, $1377,1349,1282,1231,1210,1157,751,690 ;{ }^{1} \mathrm{H}$ NMR $\left(\mathrm{CDCl}_{3}, 400 \mathrm{MHz}\right) \delta$ 7.05-7.71 (m, 10H, Ph-H), 6.11 (s, $1 \mathrm{H}, \mathrm{OH}), 2.47\left(\mathrm{t}, 2 \mathrm{H}, J 7.4 \mathrm{~Hz}, \mathrm{CH}_{2}\right), 1.60-1.68(\mathrm{~m}, 2 \mathrm{H}$, $\left.\mathrm{CH}_{2}\right), 0.90\left(\mathrm{t}, 3 \mathrm{H}, J 7.4 \mathrm{~Hz}, \mathrm{CH}_{3}\right) ;{ }^{13} \mathrm{C} \mathrm{NMR}\left(\mathrm{CDCl}_{3}, 100\right.$ MHz) $\delta 185.9,177.2,145.7,137.7,133.8,130.7$ (3C), 129.1 (2C), 128.1 (2C), 123.7, 118.8 (2C), 41.0, 18.8, 13.9; Anal. calcd. for $\mathrm{C}_{18} \mathrm{H}_{19} \mathrm{~N}_{3} \mathrm{OS}_{2}$ : C, 60.47; H, 5.36; N, 11.75; Found C, 60.50; H, 5.35; N, 11.74 .

6-Hydroxy-6-butyl-1,5-diphenyl-1,3,5-triazinane2,4-dithione (4)

White solid, mp $155-157^{\circ} \mathrm{C}$; IR (KBr) $v_{\text {max }} / \mathrm{cm}^{-1} 3418$, 3059, 2949, 2864, 1617, 1602, 1585, 1541, 1477, 1443, 1349, 1290, 1204, 752, 694; ${ }^{1} \mathrm{H} \mathrm{NMR}\left(\mathrm{CDCl}_{3}, 400 \mathrm{MHz}\right)$ $\delta$ 7.05-7.71 (m, 10H, Ph-H), $6.11(\mathrm{~s}, 1 \mathrm{H}, \mathrm{OH}), 2.49(\mathrm{t}, 2 \mathrm{H}$, J 7.4 Hz, $\left.\mathrm{CH}_{2}\right), 1.57-1.64\left(\mathrm{~m}, 2 \mathrm{H}, \mathrm{CH}_{2}\right), 1.26-1.33(\mathrm{~m}, 2 \mathrm{H}$, $\left.\mathrm{CH}_{2}\right), 0.87\left(\mathrm{t}, 3 \mathrm{H}, J 7.4 \mathrm{~Hz}, \mathrm{CH}_{3}\right) ;{ }^{13} \mathrm{C}$ NMR $\left(\mathrm{CDCl}_{3}, 100\right.$ MHz) $\delta 186.1,177.2,145.7,137.7,133.8,130.7$ (3C), 129.1 (2C), 128.1 (2C), 123.7, 118.8 (2C), 38.8, 27.5, 22.4, 13.9; Anal. calcd. for $\mathrm{C}_{19} \mathrm{H}_{21} \mathrm{~N}_{3} \mathrm{OS}_{2}$ : C, 61.42; $\mathrm{H}, 5.70 ; \mathrm{N}$, 11.31; Found C, 61.54; H, 5.71; N, 11.29.

6-Hydroxy-6-pentyl-1,5-diphenyl-1,3,5-triazinane2,4-dithione (5)

White solid, mp 124-126 ${ }^{\circ} \mathrm{C}$; IR (KBr) $v_{\text {max }} / \mathrm{cm}^{-1} 3448$, 2955, 2927, 2864, 1616, 1578, 1539, 1513, 1480, 1441, $1350,1245,1201,752,692 ;{ }^{1} \mathrm{H} \mathrm{NMR}\left(\mathrm{CDCl}_{3}, 400 \mathrm{MHz}\right)$ $\delta$ 7.05-7.70 (m, 10H, Ph-H), $6.12(\mathrm{~s}, 1 \mathrm{H}, \mathrm{OH}), 2.48(\mathrm{t}, 2 \mathrm{H}$, $\left.J 7.4 \mathrm{~Hz}, \mathrm{CH}_{2}\right), 1.60-1.64\left(\mathrm{~m}, 2 \mathrm{H}, \mathrm{CH}_{2}\right), 1.26-1.27(\mathrm{~m}, 4 \mathrm{H}$, $\left.\mathrm{CH}_{2}\right), 0.85$ (t, $\left.3 \mathrm{H}, J 7.4 \mathrm{~Hz}, \mathrm{CH}_{3}\right) ;{ }^{13} \mathrm{C}$ NMR $\left(\mathrm{CDCl}_{3}, 100\right.$ MHz) $\delta 186.1,177.2,145.7,137.7,133.8,130.7$ (3C), 129.1 (2C), 128.1 (2C), 123.7, 118.8 (2C), 39.0, 31.5, 25.0, 22.4, 13.9; Anal. calcd. for $\mathrm{C}_{20} \mathrm{H}_{23} \mathrm{~N}_{3} \mathrm{OS}_{2}$ : C, 62.30; $\mathrm{H}, 6.01$; N, 10.90; Found C, 62.22; H, 5.99; N, 10.88 . 
6-Hydroxy-6-hexyl-1,5-diphenyl-1,3,5-triazinane2,4-dithione (6)

White solid, mp 105-106 ${ }^{\circ} \mathrm{C}$; IR (KBr) $v_{\max } / \mathrm{cm}^{-1}: 3450$, 2945, 2921, 2850, 1614, 1577, 1539, 1481, 1439, 1348, 1299, 1240, 1199, 752, 692; ${ }^{1} \mathrm{H}$ NMR $\left(\mathrm{CDCl}_{3}, 400 \mathrm{MHz}\right)$ $\delta$ 7.05-7.71 (m, 10H, Ph- $\mathrm{H}), 6.11(\mathrm{~s}, 1 \mathrm{H}, \mathrm{OH}), 2.48(\mathrm{t}, 2 \mathrm{H}$, $\left.J 7.4 \mathrm{~Hz}, \mathrm{CH}_{2}\right), 1.58-1.63\left(\mathrm{~m}, 2 \mathrm{H}, \mathrm{CH}_{2}\right), 1.22-1.31(\mathrm{~m}$, $\left.6 \mathrm{H}, \mathrm{CH}_{2}\right), 0.85\left(\mathrm{t}, 3 \mathrm{H}, J 7.4 \mathrm{~Hz}, \mathrm{CH}_{3}\right) ;{ }^{13} \mathrm{C} \mathrm{NMR}\left(\mathrm{CDCl}_{3}\right.$, $100 \mathrm{MHz}) \delta 186.1,177.2,145.7,137.7,133.8,130.7(2 \mathrm{C})$, 129.1 (2C), $128.5(2 \mathrm{C}), 128.1,123.7,118.8(2 \mathrm{C}), 39.1,31.6$, 29.0, 25.3, 22.5, 14.0; Anal. calcd. for $\mathrm{C}_{21} \mathrm{H}_{25} \mathrm{~N}_{3} \mathrm{OS}_{2}$ : C, 63.12; H, 6.31; N,10.52; Found C, 63.01; H, 6.30; N, 10.47.

6-Hydroxy-6-methyl-1,5-di(2-methylphenyl)1,3,5-triazinane-2,4-dithione (7)

White solid, mp 239-241 ${ }^{\circ} \mathrm{C}$; IR (KBr) $v_{\max } / \mathrm{cm}^{-1} 3415$, 2961, 2925, 2856, 1617, 1587, 1544, 1484, 1455, 1363, 1300, 1256,$752 ;{ }^{1} \mathrm{HNMR}\left(\mathrm{CDCl}_{3}, 400 \mathrm{MHz}\right) \delta 8.11(\mathrm{~d}, 1 \mathrm{H}, J 8.4 \mathrm{~Hz}$, $\mathrm{Ph}-\mathrm{H}), 7.00-7.59$ (m, 7H, Ph-H), 5.89 (s, 1H, OH), 2.24 (s, $\left.3 \mathrm{H}, \mathrm{CH}_{3}\right), 2.20$ (s, $\left.3 \mathrm{H}, \mathrm{CH}_{3}\right), 1.88\left(\mathrm{~s}, 3 \mathrm{H}, \mathrm{CH}_{3}\right) ;{ }^{13} \mathrm{C} \mathrm{NMR}$ $\left(\mathrm{CDCl}_{3}, 100 \mathrm{MHz}\right) \delta 183.3,176.9,145.8,136.9,135.9$, 132.7, 132.4, 131.3, 130.5, 128.3, 128.1, 127.2, 126.7, 124.1, 119.9, 26.1, 17.5, 16.9; Anal. calcd. for $\mathrm{C}_{18} \mathrm{H}_{19} \mathrm{~N}_{3} \mathrm{OS}_{2}$ : C, $60.47 ; \mathrm{H}, 5.36 ; \mathrm{N}, 11.75$; Found C, 60.53; H, 5.35; N, 11.71.

6-Hydroxy-6-ethyl-1,5-di(2-methylphenyl)1,3,5-triazinane-2,4-dithione ( 8 )

White solid, mp 160-162 ${ }^{\circ} \mathrm{C}$; IR (KBr) $v_{\max } / \mathrm{cm}^{-1} 3413$, 3061, 2972, 2925, 2860, 1618, 1587, 1549, 1489, 1456, 1371, 1226,$748 ;{ }^{1} \mathrm{HNMR}\left(\mathrm{CDCl}_{3}, 400 \mathrm{MHz}\right) \delta 8.10(\mathrm{~d}, 1 \mathrm{H}, J 8.0 \mathrm{~Hz}$, $\mathrm{Ph}-\mathrm{H}), 6.97-7.58(\mathrm{~m}, 7 \mathrm{H}, \mathrm{Ph}-\mathrm{H}), 5.90(\mathrm{~s}, 1 \mathrm{H}, \mathrm{OH}), 2.53(\mathrm{q}, 2 \mathrm{H}$, $\left.J 7.4 \mathrm{~Hz}, \mathrm{CH}_{2}\right), 2.17\left(\mathrm{~s}, 3 \mathrm{H}, \mathrm{CH}_{3}\right), 1.88\left(\mathrm{~s}, 3 \mathrm{H}, \mathrm{CH}_{3}\right), 1.13$ (t, $\left.3 \mathrm{H}, J 7.4 \mathrm{~Hz}, \mathrm{CH}_{3}\right) ;{ }^{13} \mathrm{C} \mathrm{NMR}\left(\mathrm{CDCl}_{3}, 100 \mathrm{MHz}\right) \delta 186.7$, 176.6, 145.8, 137.0, 135.9, 132.7, 132.3, 131.2, 130.5, 128.2, 128.1, 127.2, 126.7, 124.1, 119.9, 32.3, 17.5, 16.9, 9.5; Anal. calcd. for $\mathrm{C}_{19} \mathrm{H}_{21} \mathrm{~N}_{3} \mathrm{OS}_{2}: \mathrm{C}, 61.42 ; \mathrm{H}, 5.70 ; \mathrm{N}, 11.31$; Found C, 61.47; H, 5.69; N, 11.28.

6-Hydroxy-6-propyl-1,5-di(2-methylphenyl)1,3,5-triazinane-2,4-dithione (9)

White solid, mp $154-155{ }^{\circ} \mathrm{C}$; IR (KBr) $v_{\max } / \mathrm{cm}^{-1} 3409$, 3009, 2963, 2928, 2841, 1609, 1547, 1511, 1468, 1366, 1300, 1254,$668 ;{ }^{1} \mathrm{HNMR}\left(\mathrm{CDCl}_{3}, 400 \mathrm{MHz}\right) \delta 8.10(\mathrm{~d}, 1 \mathrm{H}, J 8.4 \mathrm{~Hz}$, $\mathrm{Ph}-\mathrm{H}), 7.00-7.56(\mathrm{~m}, 7 \mathrm{H}, \mathrm{Ph}-\mathrm{H}), 5.89$ (s, 1H, OH), 2.47 (t, 2H, $\left.J 7.6 \mathrm{~Hz}, \mathrm{CH}_{2}\right), 2.20\left(\mathrm{~s}, 3 \mathrm{H}, \mathrm{CH}_{3}\right), 1.88\left(\mathrm{~s}, 3 \mathrm{H}, \mathrm{CH}_{3}\right), 1.62-$ $1.68\left(\mathrm{~m}, 2 \mathrm{H}, \mathrm{CH}_{2}\right), 0.90\left(\mathrm{t}, 3 \mathrm{H}, J 7.2 \mathrm{~Hz}, \mathrm{CH}_{3}\right) ;{ }^{13} \mathrm{C} \mathrm{NMR}$ $\left(\mathrm{CDCl}_{3}, 100 \mathrm{MHz}\right) \delta 186.0,176.6,145.8,137.0,135.9$, 132.7, 132.3, 131.2, 130.5, 128.2, 128.1, 127.2, 126.7, 124.1, 119.9, 41.0, 18.9, 17.5, 16.9, 13.8; Anal. calcd. for $\mathrm{C}_{20} \mathrm{H}_{23} \mathrm{~N}_{3} \mathrm{OS}_{2}: \mathrm{C}, 62.30 ; \mathrm{H}, 6.01 ; \mathrm{N}, 10.90$; Found C, 62.15; $\mathrm{H}, 5.99 ; \mathrm{N}, 10.87$.
6-Hydroxy-6-butyl-1,5-di(2-methylphenyl)1,3,5-triazinane-2,4-dithione (10)

White solid, mp 123-124 ${ }^{\circ} \mathrm{C}$; IR (KBr) $v_{\max } / \mathrm{cm}^{-1} 3383$, 2961, 2924, 2857, 1600, 1535, 1512, 1471, 1408, 1341, $1240,1201,1070,804 ;{ }^{1} \mathrm{H} \mathrm{NMR}\left(\mathrm{CDCl}_{3}, 400 \mathrm{MHz}\right) \delta 8.09$ (d, 1H, J 8.0 Hz, Ph-H), 6.99-7.56 (m, 7H, Ph-H), 5.90 (s, $1 \mathrm{H}, \mathrm{OH}), 2.49$ (t, $\left.2 \mathrm{H}, J 7.6 \mathrm{~Hz}, \mathrm{CH}_{2}\right), 2.20\left(\mathrm{~s}, 3 \mathrm{H}, \mathrm{CH}_{3}\right.$ ), $1.88\left(\mathrm{~s}, 3 \mathrm{H}, \mathrm{CH}_{3}\right), 1.57-1.65\left(\mathrm{~m}, 2 \mathrm{H}, \mathrm{CH}_{2}\right), 1.26-1.33$ (m, $\left.2 \mathrm{H}, \mathrm{CH}_{2}\right), 0.87$ (t, 3H, J $\left.7.2 \mathrm{~Hz}, \mathrm{CH}_{3}\right) ;{ }^{13} \mathrm{C} \mathrm{NMR}\left(\mathrm{CDCl}_{3}\right.$, $100 \mathrm{MHz}) 186.1,176.5,145.8,137.0,135.9,132.6,132.3$, 131.2, 130.4, 128.2, 128.0, 127.1, 126.7, 124.1, 119.9, 38.8, 27.5, 22.4, 17.4, 16.9, 13.8; Anal. calcd. for $\mathrm{C}_{21} \mathrm{H}_{25} \mathrm{~N}_{3} \mathrm{OS}_{2}$ : C, 63.12; H, 6.31; N, 10.52; Found C, 63.05; H, 6.30; N, 10.49 .

6-Hydroxy-6-ethyl-1,5-di(4-methylphenyl)1,3,5-triazinane-2,4-dithione (11)

White solid, mp 119-121 ${ }^{\circ} \mathrm{C}$; IR (KBr) $v_{\max } / \mathrm{cm}^{-1} 3396$, 3264, 3041, 2962, 2925, 2857, 1600, 1583, 1534, 1513, 1472, $1454,1370,1223,1062,810 ;{ }^{1} \mathrm{H} \mathrm{NMR}\left(\mathrm{CDCl}_{3}, 400 \mathrm{MHz}\right) \delta$ 7.44 (d, 2H, J 8.0 Hz, Ph-H), 7.26-7.33 (m, 4H, Ph-H), 7.10 (d, $2 \mathrm{H}, J 8.0 \mathrm{~Hz}, \mathrm{Ph}-\mathrm{H}), 6.07$ (s, 1H, OH), 2.51 (s, 3H, $\mathrm{CH}_{3}$ ), $2.51\left(\mathrm{q}, 2 \mathrm{H}, J 7.4 \mathrm{~Hz}, \mathrm{CH}_{2}\right), 2.29\left(\mathrm{~s}, 3 \mathrm{H}, \mathrm{CH}_{3}\right), 1.12(\mathrm{t}, 3 \mathrm{H}$, $\left.J 7.4 \mathrm{~Hz}, \mathrm{CH}_{3}\right) ;{ }^{13} \mathrm{C} \mathrm{NMR}\left(\mathrm{CDCl}_{3}, 100 \mathrm{MHz}\right) \delta 186.5,177.5$, 146.2, 141.0, 135.2, 133.3, 131.3 (2C), 131.0, 129.6 (2C), 127.8 (2C), 119.1 (2C), 32.3, 21.4, 20.7, 9.5; Anal. calcd. for $\mathrm{C}_{19} \mathrm{H}_{21} \mathrm{~N}_{3} \mathrm{OS}_{2}$ : C, 61.42; H, 5.70; N, 11.31; Found C, 61.38; H, 5.70; N, 11.29.

6-Hydroxy-6-propyl-1,5-di(4-methylphenyl)1,3,5-triazinane-2,4-dithione (12)

White solid, mp 127-129 ${ }^{\circ} \mathrm{C}$; IR (KBr) $v_{\max } / \mathrm{cm}^{-1} 3412$, 3321, 3035, 2961, 2925, 2870, 1604, 1539, 1512, 1459, $1409,1377,1279,1208,1067,813 ;{ }^{1} \mathrm{H}$ NMR $\left(\mathrm{CDCl}_{3}, 400\right.$ MHz) $\delta 7.44$ (d, 2H, $J 8.0 \mathrm{~Hz}, \mathrm{Ph}-\mathrm{H}), 7.26-7.33(\mathrm{~m}, 4 \mathrm{H}$, $\mathrm{Ph}-\mathrm{H}$ ), 7.10 (d, 2H, J 8.0 Hz, Ph-H), 6.09 (s, 1H, OH), 2.49 (s, 3H, $\left.\mathrm{CH}_{3}\right), 2.46$ (t, $2 \mathrm{H}, J 7.4 \mathrm{~Hz}, \mathrm{CH}_{2}$ ), 2.29 (s, $3 \mathrm{H}$, $\left.\mathrm{CH}_{3}\right), 1.61-1.67\left(\mathrm{~m}, 2 \mathrm{H}, \mathrm{CH}_{2}\right), 0.89$ (t, $3 \mathrm{H}, J 7.4 \mathrm{~Hz}, \mathrm{CH}_{3}$ ); ${ }^{13} \mathrm{C} \mathrm{NMR}\left(\mathrm{CDCl}_{3}, 100 \mathrm{MHz}\right) \delta 185.7,177.4,146.2,141.0$, 135.2, 133.3, 131.3 (2C), 131.0, 129.5 (2C), 127.7 (2C), 119.1 (2C), 41.0, 21.4, 20.7, 18.8, 13.8; Anal. calcd. for $\mathrm{C}_{20} \mathrm{H}_{23} \mathrm{~N}_{3} \mathrm{OS}_{2}$ : C, 62.30; H, 6.01; N, 10.90; Found C, 62.37; $\mathrm{H}, 6.00 ; \mathrm{N}, 10.87$.

6-Hydroxy-6-butyl-1,5-di(4-methylphenyl)1,3,5-triazinane-2,4-dithione (13)

White solid, mp 126- $127^{\circ} \mathrm{C}$; IR (KBr) $v_{\max } / \mathrm{cm}^{-1} 3425$, 3032, 2958, 2924, 2857, 1599, 1581, 1533, 1508, 1458, $1350,1282,1244,1209,810 ;{ }^{1} \mathrm{H}$ NMR $\left(\mathrm{CDCl}_{3}, 400 \mathrm{MHz}\right)$ $\delta 7.43$ (d, 2H, J 8.0 Hz, Ph-H), 7.25-7.33 (m, 4H, Ph-H), 7.09 (d, 2H, J 8.0 Hz, Ph-H), 6.08 (s, 1H, OH), 2.48 (t, 
$\left.2 \mathrm{H}, J 7.6 \mathrm{~Hz}, \mathrm{CH}_{2}\right), 2.48$ (s, 3H, $\left.\mathrm{CH}_{3}\right), 2.29$ (s, 3H, $\mathrm{CH}_{3}$ ), 1.56-1.64 (m, 2H, $\left.\mathrm{CH}_{2}\right), 1.25-1.32\left(\mathrm{~m}, 2 \mathrm{H}, \mathrm{CH}_{2}\right), 0.86$ (t, $\left.3 \mathrm{H}, J 7.4 \mathrm{~Hz}, \mathrm{CH}_{3}\right) ;{ }^{13} \mathrm{C} \mathrm{NMR}\left(\mathrm{CDCl}_{3}, 100 \mathrm{MHz}\right) \delta 185.9$, 177.4, 146.2, 141.0, 135.2, 133.3, 131.3 (2C), 131.0, 129.5 (2C), 127.7 (2C), 119.1 (2C), 38.8, 27.4, 22.4, 21.4, 20.8, 13.8; Anal. calcd. for $\mathrm{C}_{21} \mathrm{H}_{25} \mathrm{~N}_{3} \mathrm{OS}_{2}: \mathrm{C}, 63.12 ; \mathrm{H}, 6.31 ; \mathrm{N}$, 10.52; Found C, 63.18; H, 6.29; N, 10.48.

6-Hydroxy-6-pentyl-1,5-di(4-methylphenyl)1,3,5-triazinane-2,4-dithione (14)

Yellowish solid, mp $111-113{ }^{\circ} \mathrm{C}$; IR $(\mathrm{KBr}) v_{\max } / \mathrm{cm}^{-1}$ 3407, 2953, 2929, 2862, 1622, 1588, 1543, 1486, 1456, $1381,1348,1287,1258,1198,753 ;{ }^{1} \mathrm{H}$ NMR $\left(\mathrm{CDCl}_{3}, 400\right.$ MHz) $\delta 7.45$ (d, 2H, J 8.4 Hz, Ph-H), 7.26-7.33 (m, 4H, $\mathrm{Ph}-\mathrm{H}), 7.10$ (d, 2H, J 8.4 Hz, Ph-H), 6.07 (s, 1H, OH), 2.50 (s, 3H, $\left.\mathrm{CH}_{3}\right), 2.48\left(\mathrm{t}, 2 \mathrm{H}, J 7.6 \mathrm{~Hz}, \mathrm{CH}_{2}\right), 2.30\left(\mathrm{~s}, 3 \mathrm{H}, \mathrm{CH}_{3}\right)$, $1.52-1.63\left(\mathrm{~m}, 2 \mathrm{H}, \mathrm{CH}_{2}\right), 1.25-1.31\left(\mathrm{~m}, 4 \mathrm{H}, \mathrm{CH}_{2}\right), 0.85(\mathrm{t}$, $\left.3 \mathrm{H}, J 7.0 \mathrm{~Hz}, \mathrm{CH}_{3}\right) ;{ }^{13} \mathrm{C} \mathrm{NMR}\left(\mathrm{CDCl}_{3}, 100 \mathrm{MHz}\right) \delta 186.0$, 177.4, 146.2, 141.0, 135.2, 133.3, 131.3 (2C), 131.1, 129.6 (2C), 127.8 (2C), 119.1 (2C), 39.0, 31.5, 25.1, 22.4, 21.4, 20.7, 13.9; Anal. calcd. for $\mathrm{C}_{22} \mathrm{H}_{27} \mathrm{~N}_{3} \mathrm{OS}_{2}: \mathrm{C}, 63.89 ; \mathrm{H}, 6.58$; N, 10.16; Found C, 63.97; H, 6.59; N, 10.12 .

6-Hydroxy-6-hexyl-1,5-di(4-methylphenyl)1,3,5-triazinane-2,4-dithione (15)

White solid, mp 103-104 ${ }^{\circ} \mathrm{C}$; IR (KBr) $v_{\text {max }} / \mathrm{cm}^{-1} 3387$, 3063, 2929, 2862, 1600, 1539, 1513, 1476, 1382, 1348, $1298,1241,1199,815 ;{ }^{1} \mathrm{H}$ NMR $\left(\mathrm{CDCl}_{3}, 400 \mathrm{MHz}\right) \delta 7.43$ (d, 2H, J 8.4 Hz, Ph-H), 7.26-7.33 (m, 4H, Ph-H), 7.10 (d, $2 \mathrm{H}, J$ J $.4 \mathrm{~Hz}, \mathrm{Ph}-\mathrm{H}), 6.07$ (s, 1H, OH), 2.49 (s, 3H, $\mathrm{CH}_{3}$ ), 2.47 (t, $\left.2 \mathrm{H}, J 7.6 \mathrm{~Hz}, \mathrm{CH}_{2}\right), 2.29\left(\mathrm{~s}, 3 \mathrm{H}, \mathrm{CH}_{3}\right), 1.59-1.63$ (m, $\left.2 \mathrm{H}, \mathrm{CH}_{2}\right), 1.21-1.28\left(\mathrm{~m}, 6 \mathrm{H}, \mathrm{CH}_{2}\right), 0.85(\mathrm{t}, 3 \mathrm{H}, J 7.4 \mathrm{~Hz}$, $\left.\mathrm{CH}_{3}\right) ;{ }^{13} \mathrm{C} \mathrm{NMR}\left(\mathrm{CDCl}_{3}, 100 \mathrm{MHz}\right) \delta 186.0,177.4,146.2$, 141.0, 135.2, 133.3, 131.3 (2C), 131.0, 129.6 (2C), 127.8 (2C), 119.1 (2C), 39.1, 31.6, 29.0, 25.3, 22.5, 21.4, 20.7, 14.1; Anal. calcd. for $\mathrm{C}_{23} \mathrm{H}_{29} \mathrm{~N}_{3} \mathrm{OS}_{2}: \mathrm{C}, 64.60 ; \mathrm{H}, 6.84 ; \mathrm{N}$, 9.83; Found C, 64.43; H, 6.83; N, 9.79.

6-Hydroxy-6-methyl-1,5-di(4-methoxyphenyl)1,3,5-triazinane-2,4-dithione (16)

White solid, mp 218-219 ${ }^{\circ} \mathrm{C}$; IR (KBr) $v_{\max } / \mathrm{cm}^{-1} 3414$, 2959, 2930, 2863, 1619, 1588, 1531, 1456, 1251, 1193, 754; ${ }^{1} \mathrm{H}$ NMR $\left(\mathrm{CDCl}_{3}, 400 \mathrm{MHz}\right) \delta$ 7.33-7.38 (m, 4H, $\mathrm{Ph}-\mathrm{H}), 7.13$ (d, 2H, J 8.8 Hz, Ph-H), 6.85 (d, 2H, J $8.8 \mathrm{~Hz}$, $\mathrm{Ph}-\mathrm{H}), 6.01$ (s, 1H, OH), 3.91 (s, 3H, $\mathrm{OCH}_{3}$ ), 3.78 (s, 3H,
$\left.\mathrm{OCH}_{3}\right), 2.22\left(\mathrm{~s}, 3 \mathrm{H}, \mathrm{CH}_{3}\right) ;{ }^{13} \mathrm{C} \mathrm{NMR}\left(\mathrm{CDCl}_{3}, 100 \mathrm{MHz}\right) \delta$ 183.1, 178.0, 161.0, 156.2, 147.0, 130.8, 129.3 (2C), 126.0, 121.4 (2C) (2C), 116.0 (2C), 114.3 (2C), 55.6, 55.5, 26.1; Anal. calcd. for $\mathrm{C}_{18} \mathrm{H}_{19} \mathrm{~N}_{3} \mathrm{O}_{3} \mathrm{~S}_{2}: \mathrm{C}, 55.51 ; \mathrm{H}, 4.92 ; \mathrm{N}, 10.79$; Found C, 55.60; H, 4.90; N, 10.74 .

6-Hydroxy-6-ethyl-1,5-di(4-methoxyphenyl)1,3,5-triazinane-2,4-dithione (17)

White solid, mp 243-245 ${ }^{\circ} \mathrm{C}$; IR (KBr) $v_{\max } / \mathrm{cm}^{-1} 3410$, 3007, 2963, 2932, 2837, 1606, 1548, 1511, 1469, 1366, $1301,1252,824 ;{ }^{1} \mathrm{H}$ NMR $\left(\mathrm{CDCl}_{3}, 400 \mathrm{MHz}\right) \delta$ 7.33-7.36 (m, 4H, Ph-H), 7.13 (d, 2H, J 8.8 Hz, Ph-H), 6.85 (d, 2H, $J 8.8 \mathrm{~Hz}, \mathrm{Ph}-\mathrm{H}), 5.98(\mathrm{~s}, 1 \mathrm{H}, \mathrm{OH}), 3.92\left(\mathrm{~s}, 3 \mathrm{H}, \mathrm{OCH}_{3}\right), 3.78$ $\left(\mathrm{s}, 3 \mathrm{H}, \mathrm{OCH}_{3}\right), 2.51\left(\mathrm{q}, 2 \mathrm{H}, J 7.4 \mathrm{~Hz}, \mathrm{CH}_{2}\right), 1.13(\mathrm{t}, 3 \mathrm{H}, J$ $\left.7.4 \mathrm{~Hz}, \mathrm{CH}_{3}\right) ;{ }^{13} \mathrm{C} \mathrm{NMR}\left(\mathrm{CDCl}_{3}, 100 \mathrm{MHz}\right) \delta 186.6,177.8$, 160.9, 156.2, 147.0, 130.8, 129.3 (2C), 126.0, $121.4(2 \mathrm{C})$, 115.9 (2C), 114.3 (2C), 55.6, 55.5, 32.3, 9.5; Anal. calcd. for $\mathrm{C}_{19} \mathrm{H}_{21} \mathrm{~N}_{3} \mathrm{O}_{3} \mathrm{~S}_{2}: \mathrm{C}, 56.55 ; \mathrm{H}, 5.25 ; \mathrm{N}, 10.41$; Found $\mathrm{C}$, 56.46; H, 5.26; N, 10.37 .

6-Hydroxy-6-propyl-1,5-di(4-methoxyphenyl)1,3,5-triazinane-2,4-dithione (18)

White solid, mp 219-221 ${ }^{\circ} \mathrm{C}$; IR (KBr) $v_{\max } / \mathrm{cm}^{-1} 3369$, 3008, 2961, 2835, 1599, 1543, 1510, 1471, 1379, 1246, 1170, 820; ${ }^{1} \mathrm{H}$ NMR $\left(\mathrm{CDCl}_{3}, 400 \mathrm{MHz}\right) \delta$ 7.32-7.36 (m, 4H, Ph-H), 7.13 (d, 2H, J 8.8 Hz, Ph-H), 6.85 (d, 2H, J8.8 Hz, Ph-H), 6.01 (s, $1 \mathrm{H}, \mathrm{OH}), 3.91\left(\mathrm{~s}, 3 \mathrm{H}, \mathrm{OCH}_{3}\right), 3.78\left(\mathrm{~s}, 3 \mathrm{H}, \mathrm{OCH}_{3}\right), 2.46$ (t, $\left.2 \mathrm{H}, J 7.2 \mathrm{~Hz}, \mathrm{CH}_{2}\right), 1.62-1.67\left(\mathrm{~m}, 2 \mathrm{H}, \mathrm{CH}_{2}\right), 0.89(\mathrm{t}, 3 \mathrm{H}$, $\left.J 7.4 \mathrm{~Hz}, \mathrm{CH}_{3}\right) ;{ }^{13} \mathrm{C} \mathrm{NMR}\left(\mathrm{CDCl}_{3}, 100 \mathrm{MHz}\right) \delta 185.8,177.7$, 160.9, 156.2, 147.0, 130.8, 129.3 (2C), 126.0, 121.4 (2C), 115.8 (2C), 114.3 (2C), 55.6, 55.5, 41.0, 18.8, 13.8; Anal. calcd. for $\mathrm{C}_{20} \mathrm{H}_{23} \mathrm{~N}_{3} \mathrm{O}_{3} \mathrm{~S}_{2}: \mathrm{C}, 57.53 ; \mathrm{H}, 5.55 ; \mathrm{N}, 10.06$; Found C, 57.58; H, 5.57; N, 10.01 .

6-Hydroxy-6-methyl-1,5-di(4-chlorophenyl)1,3,5-triazinane-2,4-dithione (19)

White solid, mp 208-210 ${ }^{\circ} \mathrm{C}$; IR ( $\left.\mathrm{KBr}\right) v_{\max } / \mathrm{cm}^{-1} 3449$, 3286, 2960, 2925, 2856, 1605, 1539, 1491, 1450, 1373, $1300,1250,1090,825 ;{ }^{1} \mathrm{H}$ NMR $\left(\mathrm{CDCl}_{3}, 400 \mathrm{MHz}\right) \delta 7.66$ (d, $2 \mathrm{H}, J 8.4 \mathrm{~Hz}, \mathrm{Ph}-\mathrm{H}), 7.39-7.45$ (m, 4H, Ph-H), 7.27 (d, $2 \mathrm{H}, J 8.4 \mathrm{~Hz}, \mathrm{Ph}-\mathrm{H}), 6.09$ (s, 1H, OH), 2.23 (s, 3H, $\mathrm{CH}_{3}$ ); ${ }^{13} \mathrm{C} \mathrm{NMR}\left(\mathrm{CDCl}_{3}, 100 \mathrm{MHz}\right) \delta 183.3,177.3,145.1,137.2$, 136.2, 131.9, 131.2 (2C), 129.6 (2C), 129.2 (2C), 128.8, 120.1 (2C), 26.1; Anal. calcd. for $\mathrm{C}_{16} \mathrm{H}_{13} \mathrm{Cl}_{2} \mathrm{~N}_{3} \mathrm{OS}_{2}$ : C, 48.24; H, 3.29; N, 10.55; Found C, 48.10; H, 3.30; N, 10.58 . 


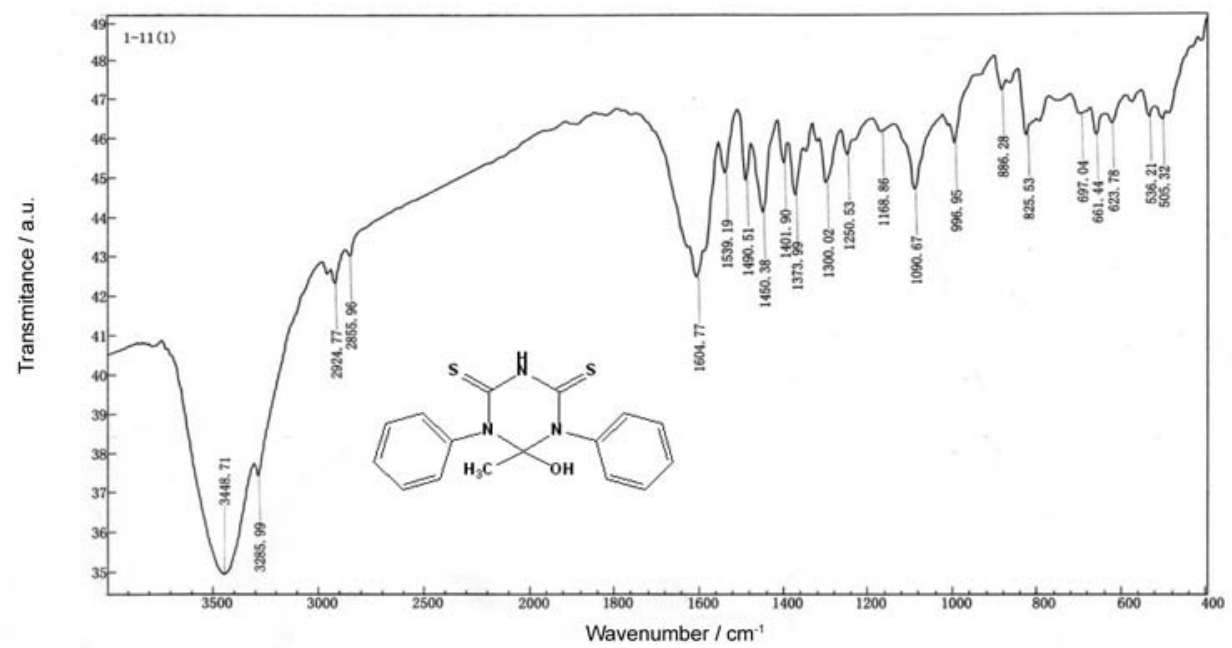

Figure S1. IR (KBr) spectrum of 6-hydroxy-6-methyl-1,5-diphenyl-1,3,5-triazinane-2,4-dithione (1).
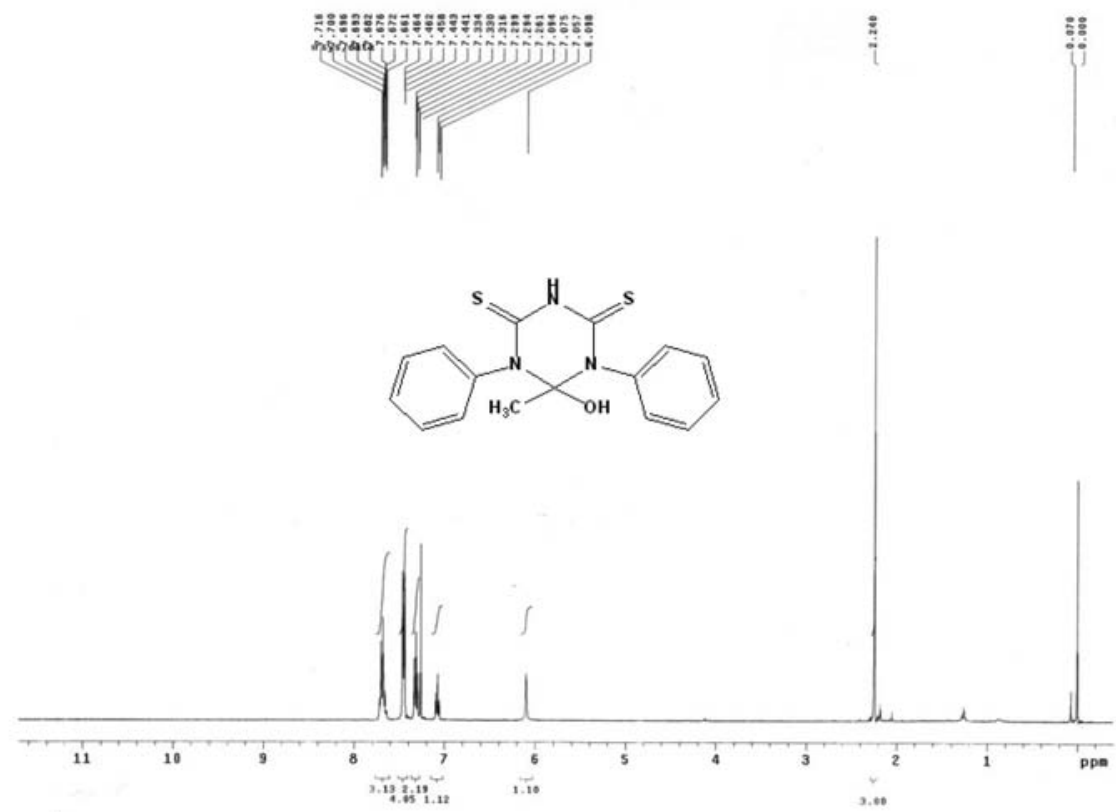

Figure S2. ${ }^{1} \mathrm{H}$ NMR $\left(\mathrm{CDCl}_{3}\right)$ spectrum of 6-hydroxy-6-methyl-1,5-diphenyl-1,3,5-triazinane-2,4-dithione (1). 

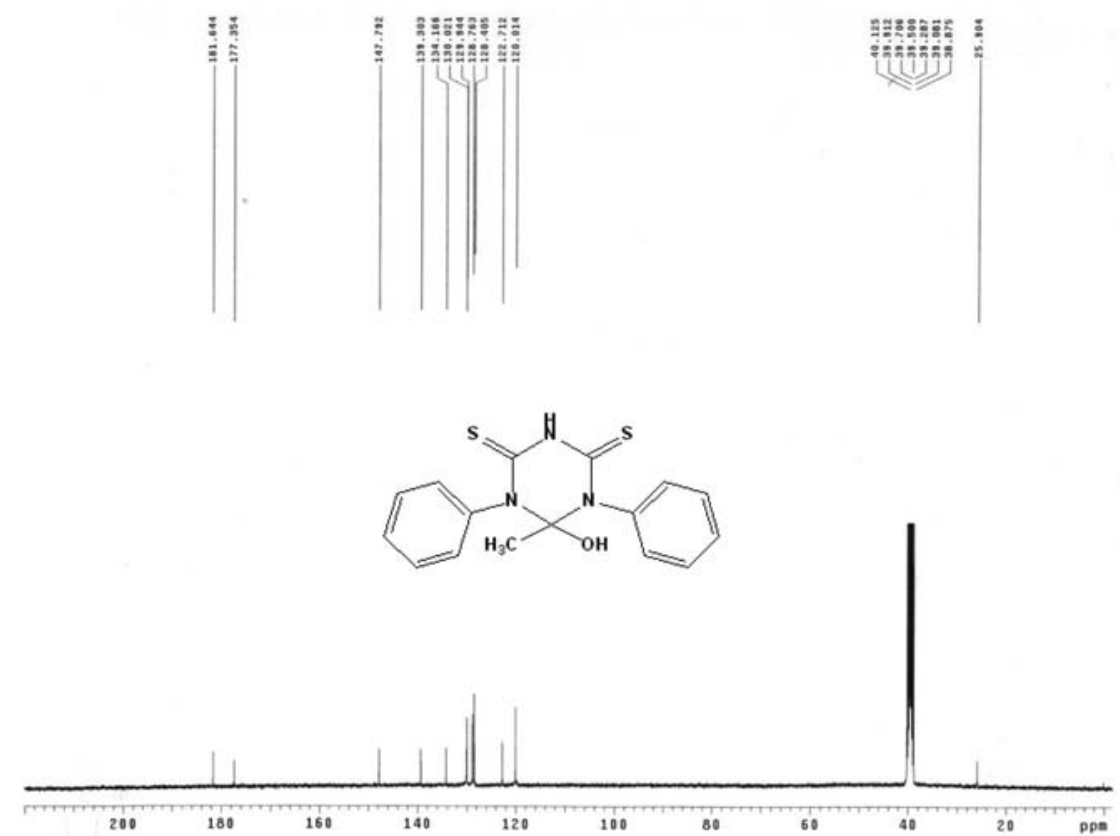

Figure S3. ${ }^{13} \mathrm{C}$ NMR (DMSO- $d_{6}$ ) spectrum of 6-hydroxy-6-methyl-1,5-diphenyl-1,3,5-triazinane-2,4-dithione (1).

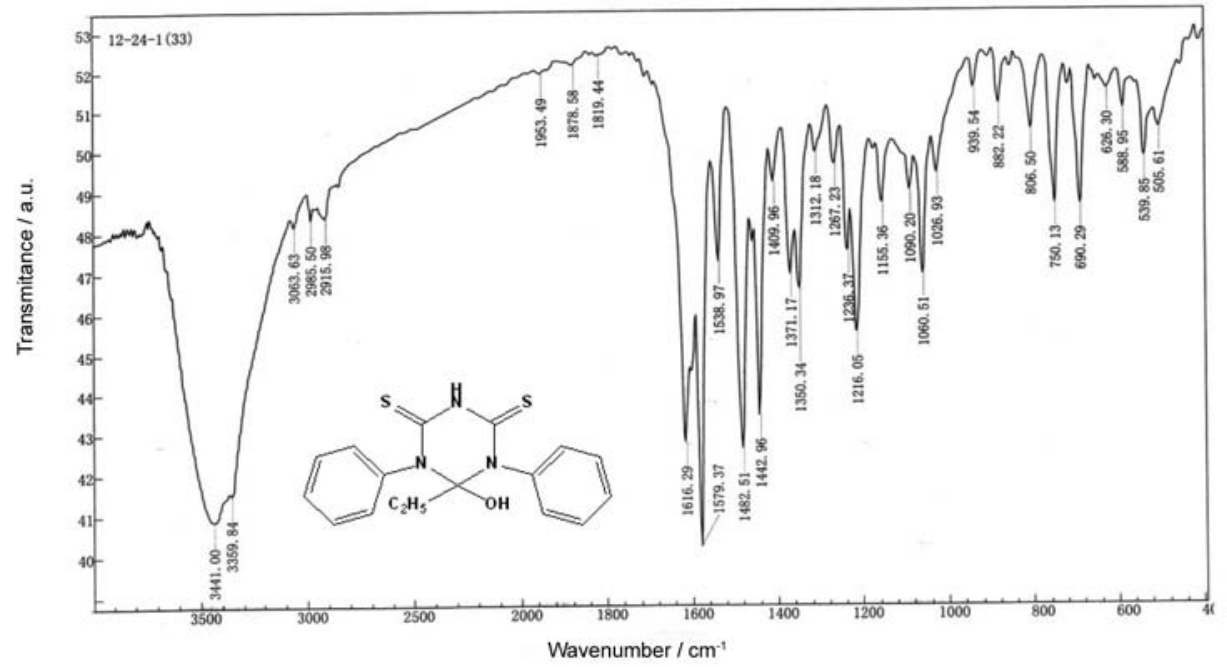

Figure S4. IR (KBr) spectrum of 6-hydroxy-6-ethyl-1,5-diphenyl-1,3,5-triazinane-2,4-dithione (2). 


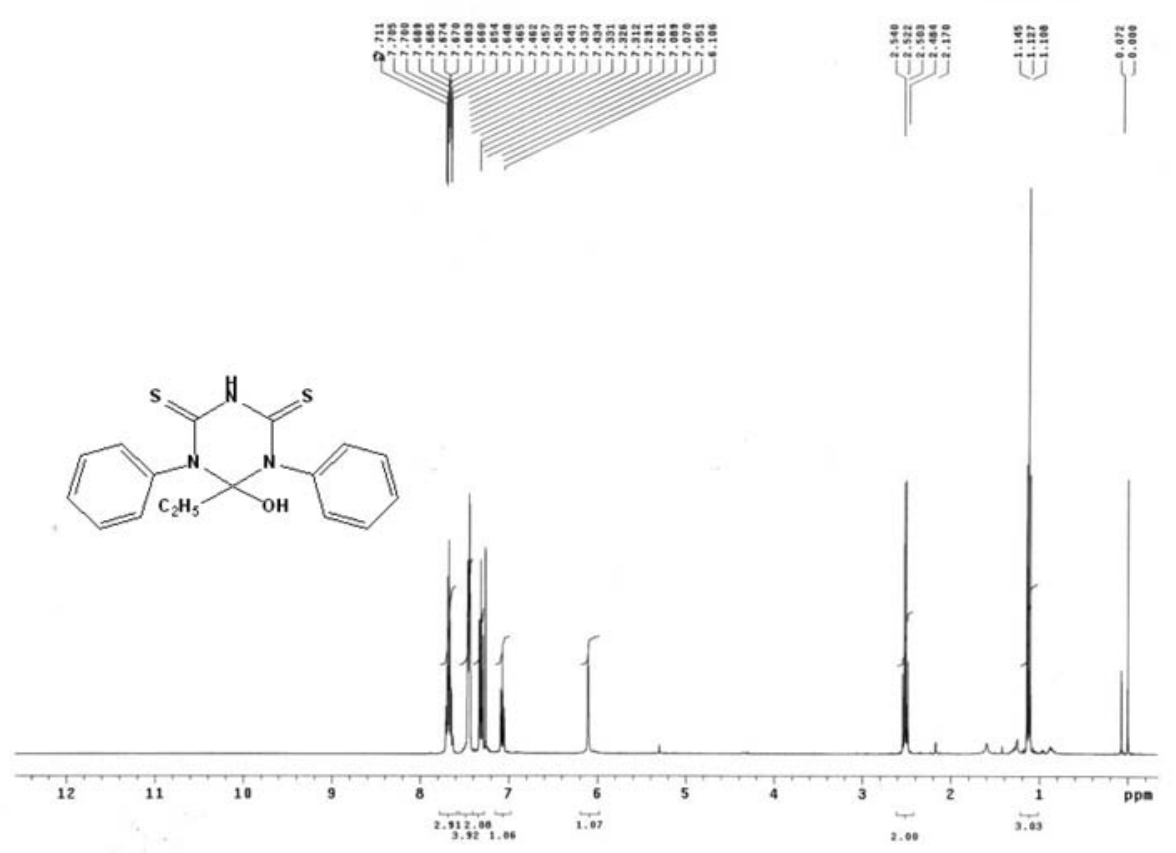

Figure S5. ${ }^{1} \mathrm{H}$ NMR $\left(\mathrm{CDCl}_{3}\right)$ spectrum of 6-hydroxy-6-ethyl-1,5-diphenyl-1,3,5-triazinane-2,4-dithione (2).
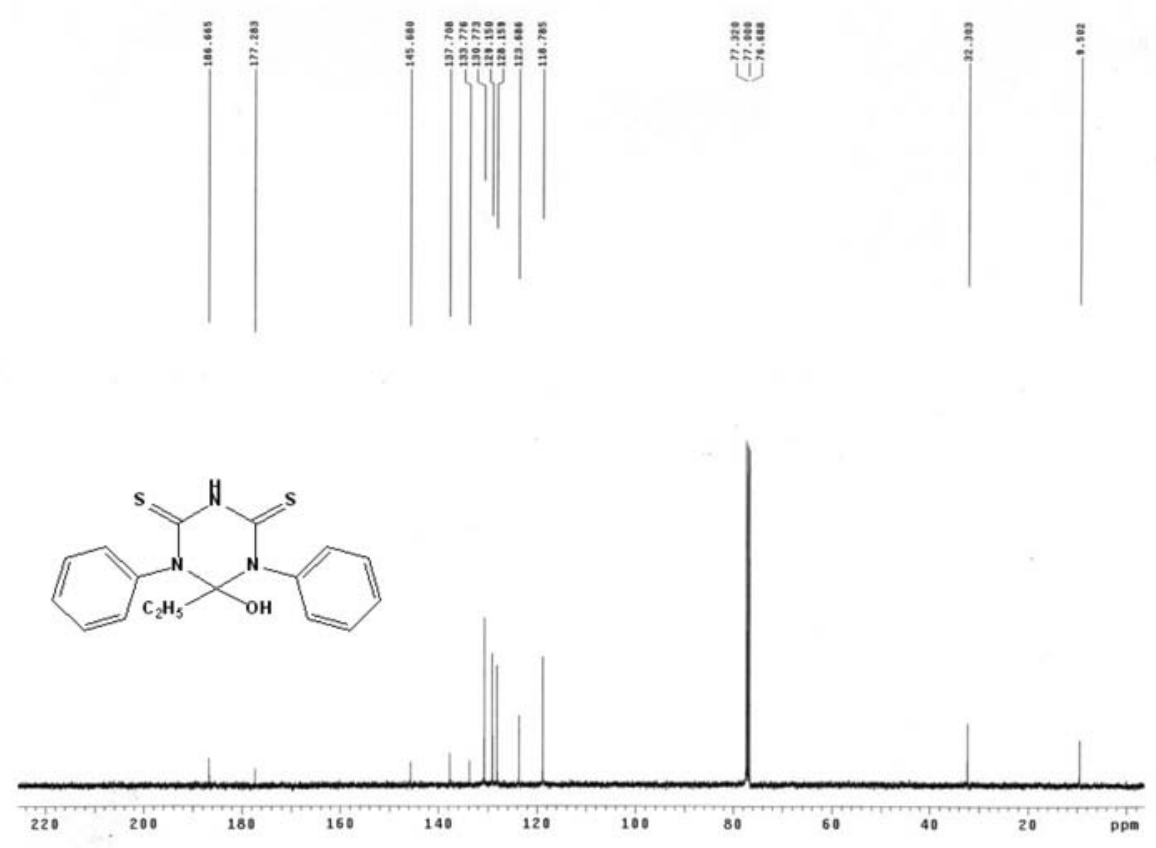

Figure S6. ${ }^{13} \mathrm{C} \mathrm{NMR}\left(\mathrm{CDCl}_{3}\right)$ spectrum of 6-hydroxy-6-ethyl-1,5-diphenyl-1,3,5-triazinane-2,4-dithione (2). 


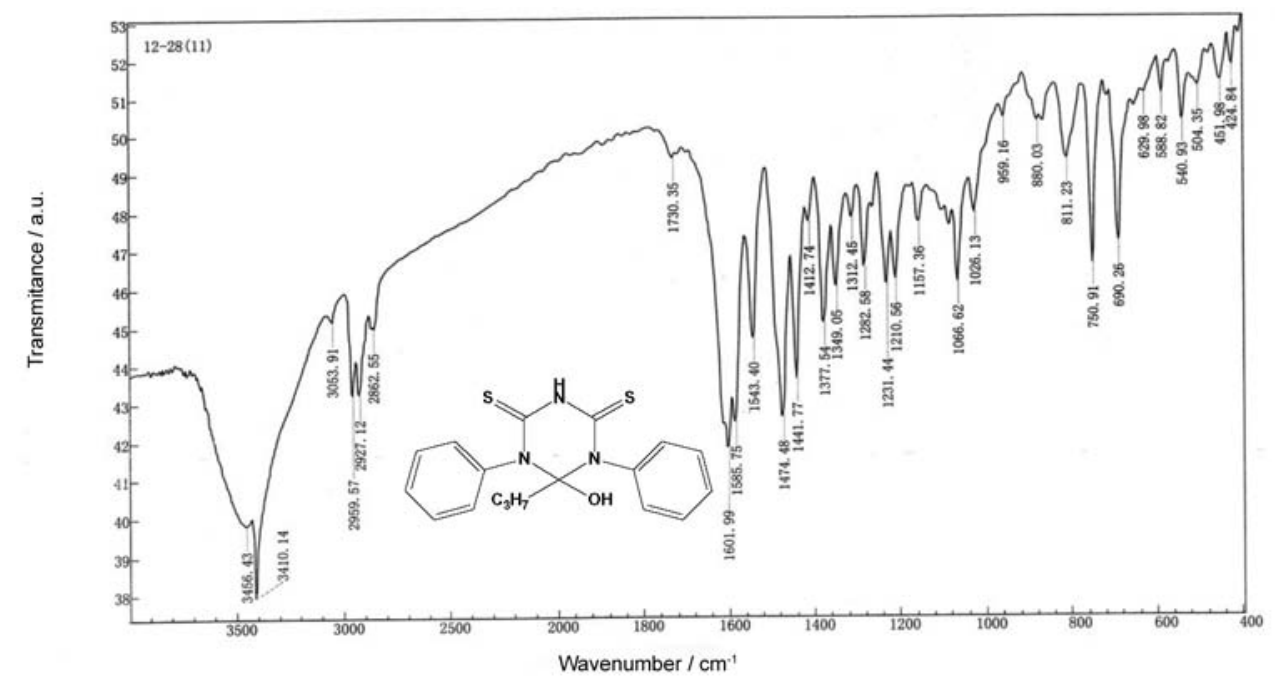

Figure S7. IR (KBr) spectrum of 6-hydroxy-6-propyl-1,5-diphenyl-1,3,5-triazinane-2,4-dithione (3).

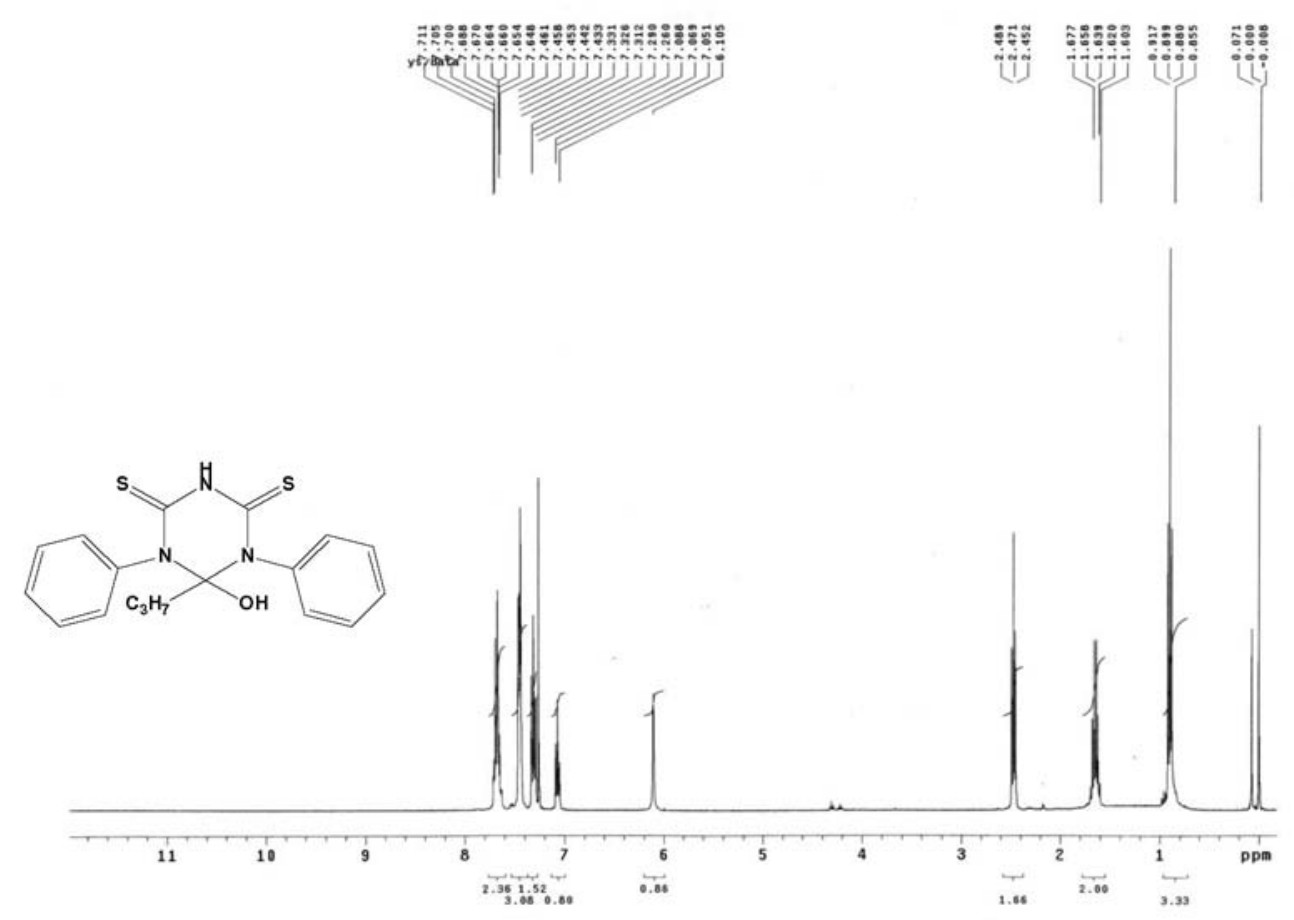

Figure S8. ${ }^{1} \mathrm{H}$ NMR $\left(\mathrm{CDCl}_{3}\right)$ spectrum of 6-hydroxy-6-propyl-1,5-diphenyl-1,3,5-triazinane-2,4-dithione (3). 

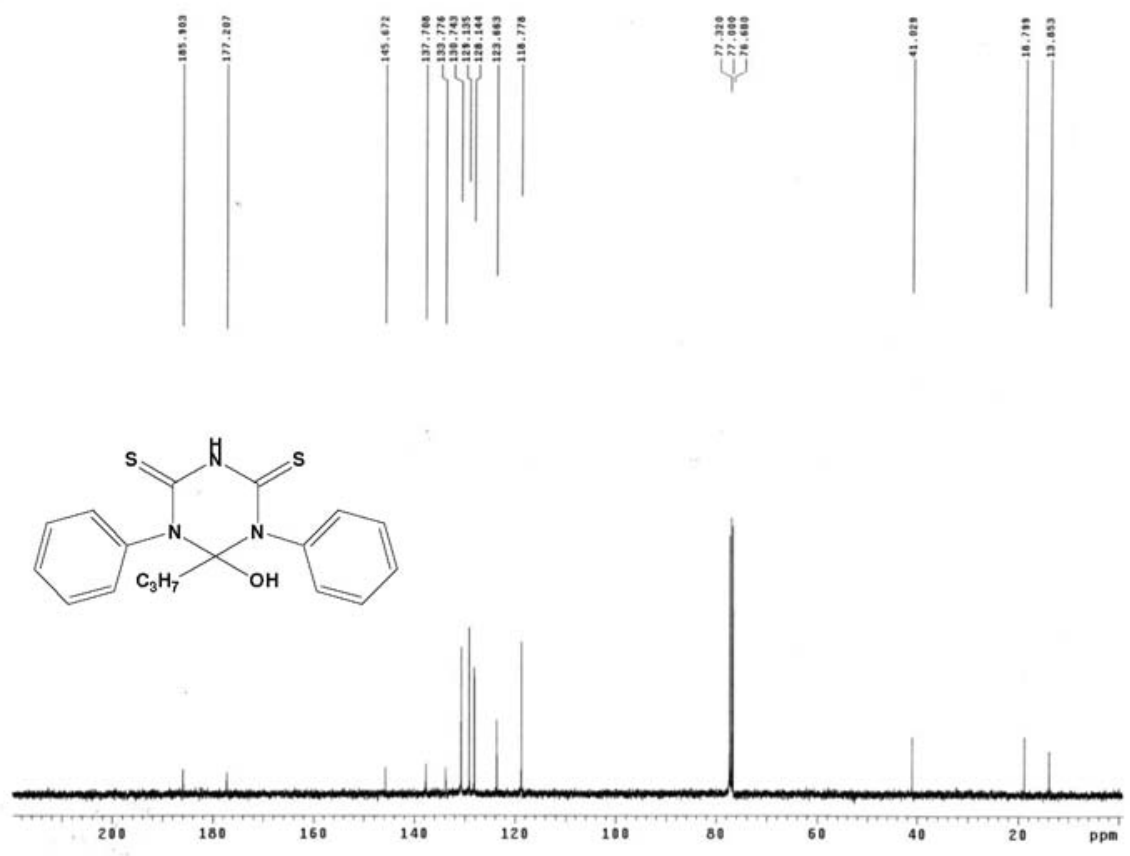

Figure S9. ${ }^{13} \mathrm{C} \mathrm{NMR}\left(\mathrm{CDCl}_{3}\right)$ spectrum of 6-hydroxy-6-propyl-1,5-diphenyl-1,3,5-triazinane-2,4-dithione (3).

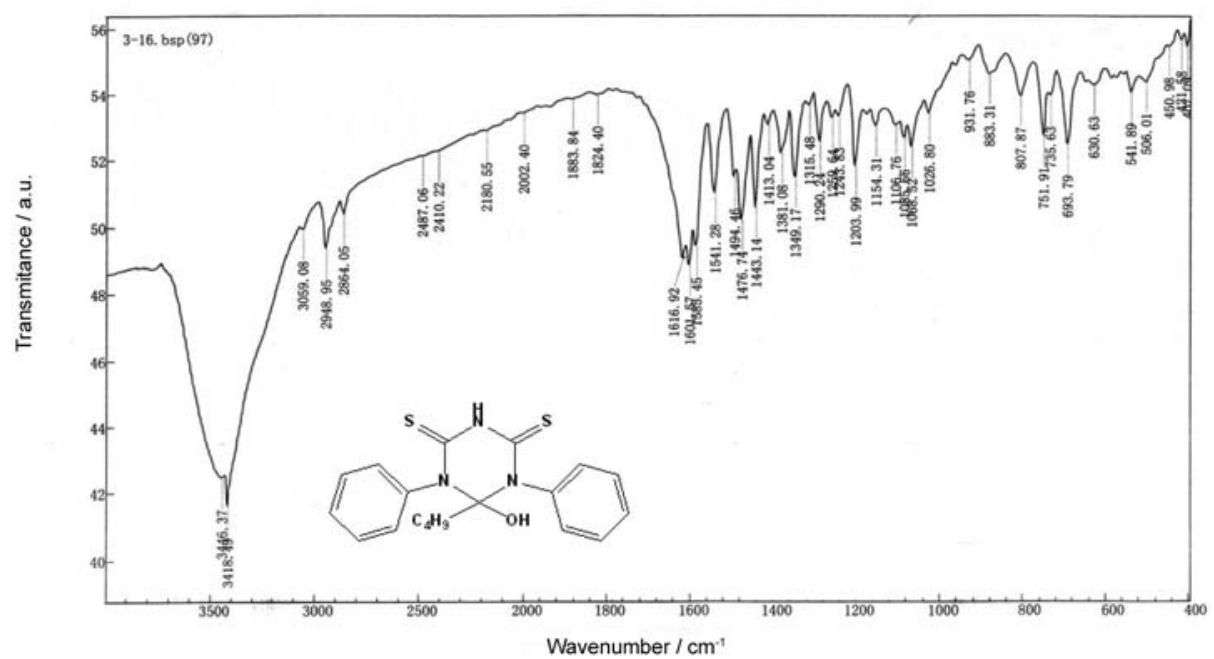

Figure S10. IR (KBr) spectrum of 6-hydroxy-6-butyl-1,5-diphenyl-1,3,5-triazinane-2,4-dithione (4). 


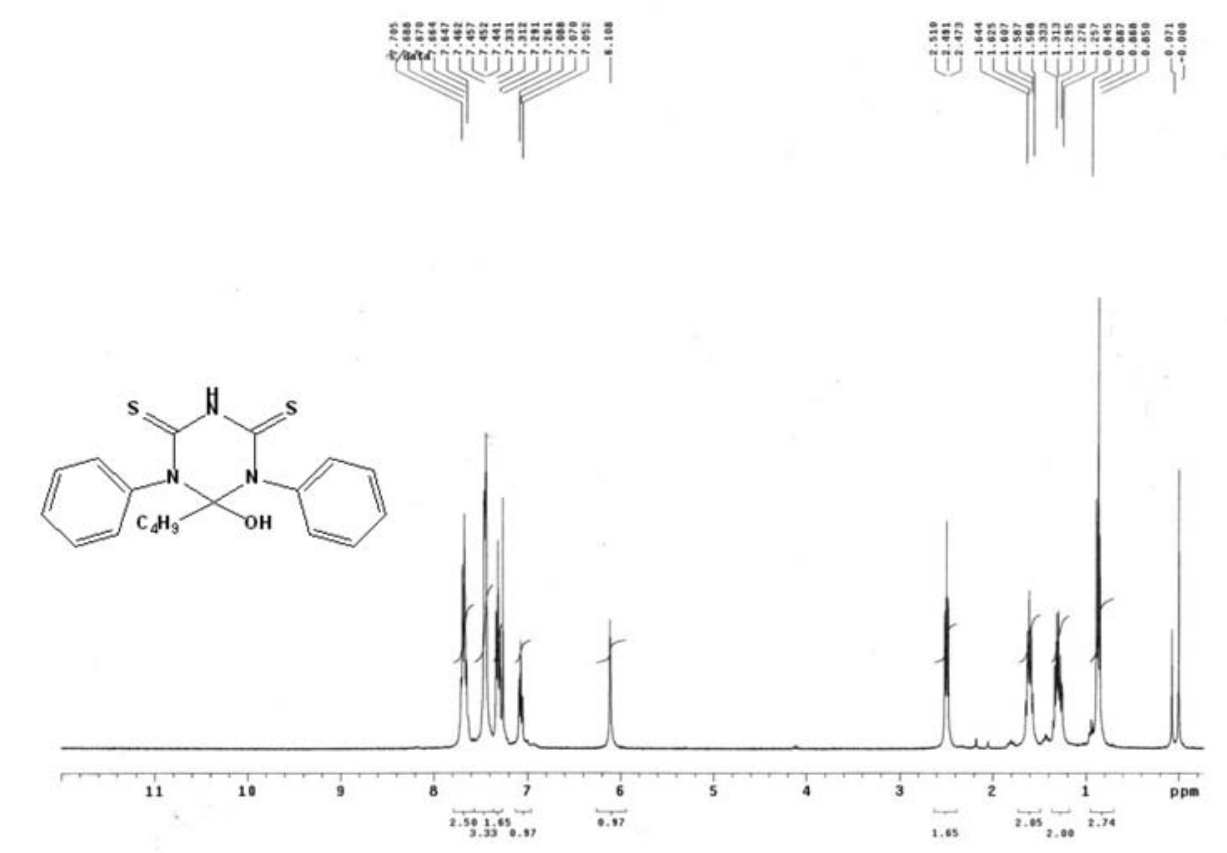

Figure S11. ${ }^{1} \mathrm{H}$ NMR $\left(\mathrm{CDCl}_{3}\right)$ spectrum of 6-hydroxy-6-butyl-1,5-diphenyl-1,3,5-triazinane-2,4-dithione (4).
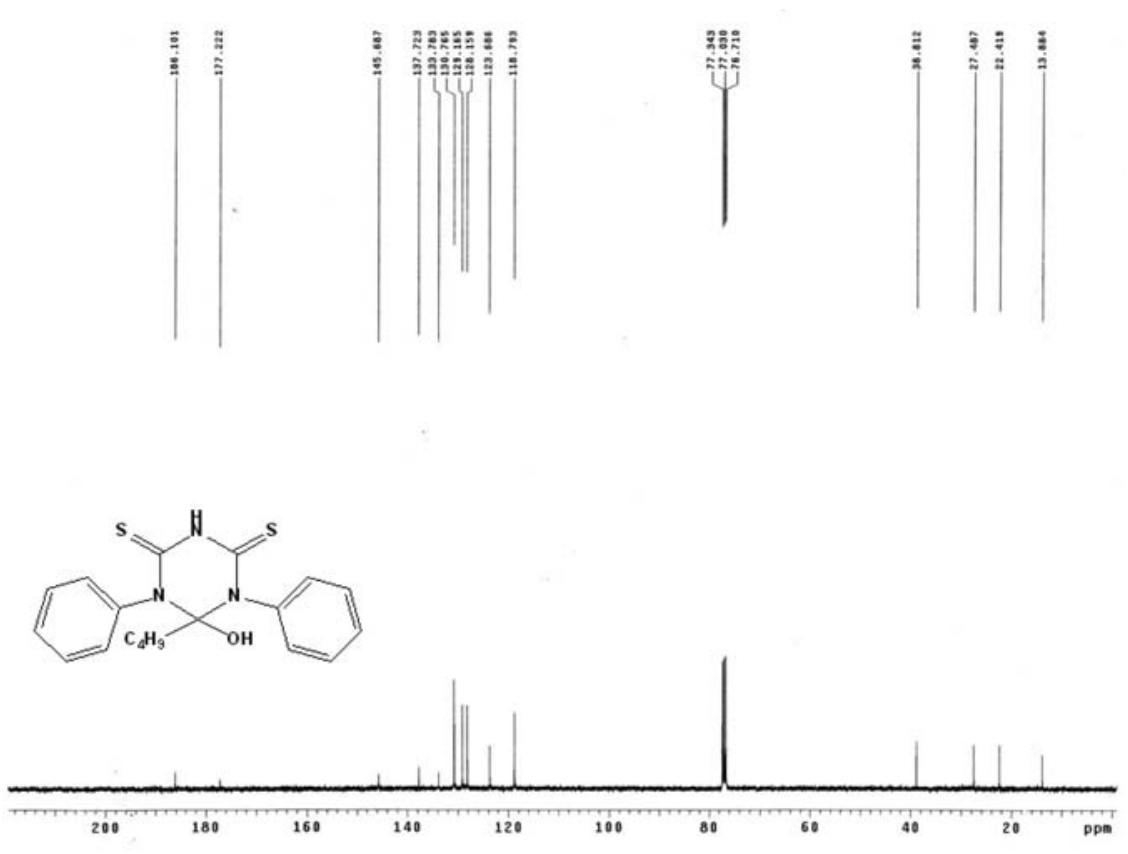

Figure S12. ${ }^{13} \mathrm{C}$ NMR $\left(\mathrm{CDCl}_{3}\right)$ spectrum of 6-hydroxy-6-butyl-1,5-diphenyl-1,3,5-triazinane-2,4-dithione (4). 


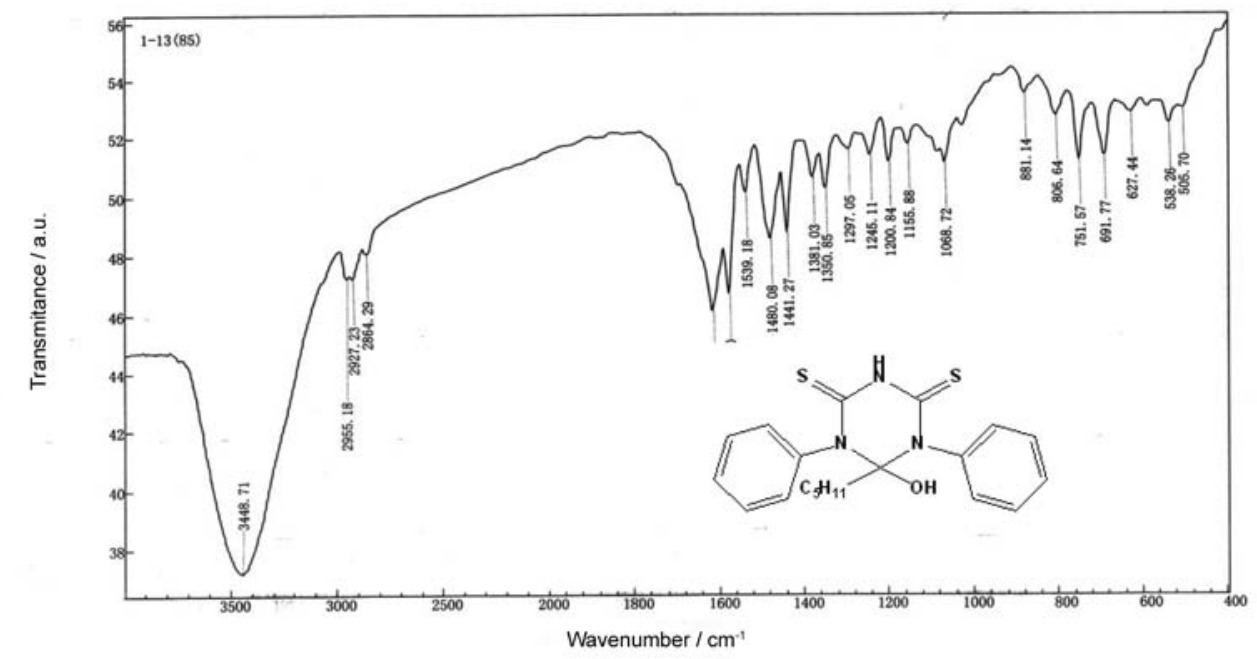

Figure S13. IR (KBr) spectrum of 6-hydroxy-6-pentyl-1,5-diphenyl-1,3,5-triazinane-2,4-dithione (5).
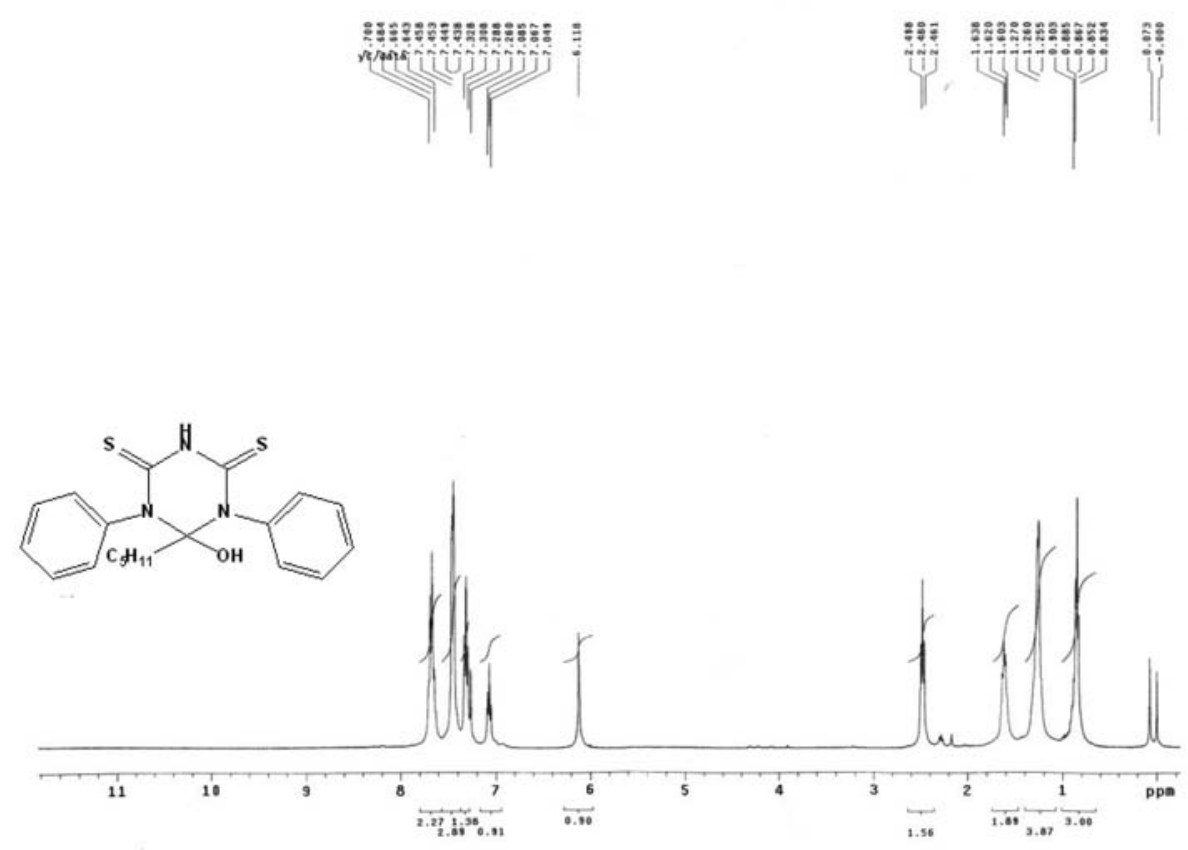

Figure S14. ${ }^{1} \mathrm{H}$ NMR $\left(\mathrm{CDCl}_{3}\right)$ spectrum of 6-hydroxy-6-pentyl-1,5-diphenyl-1,3,5-triazinane-2,4-dithione (5). 

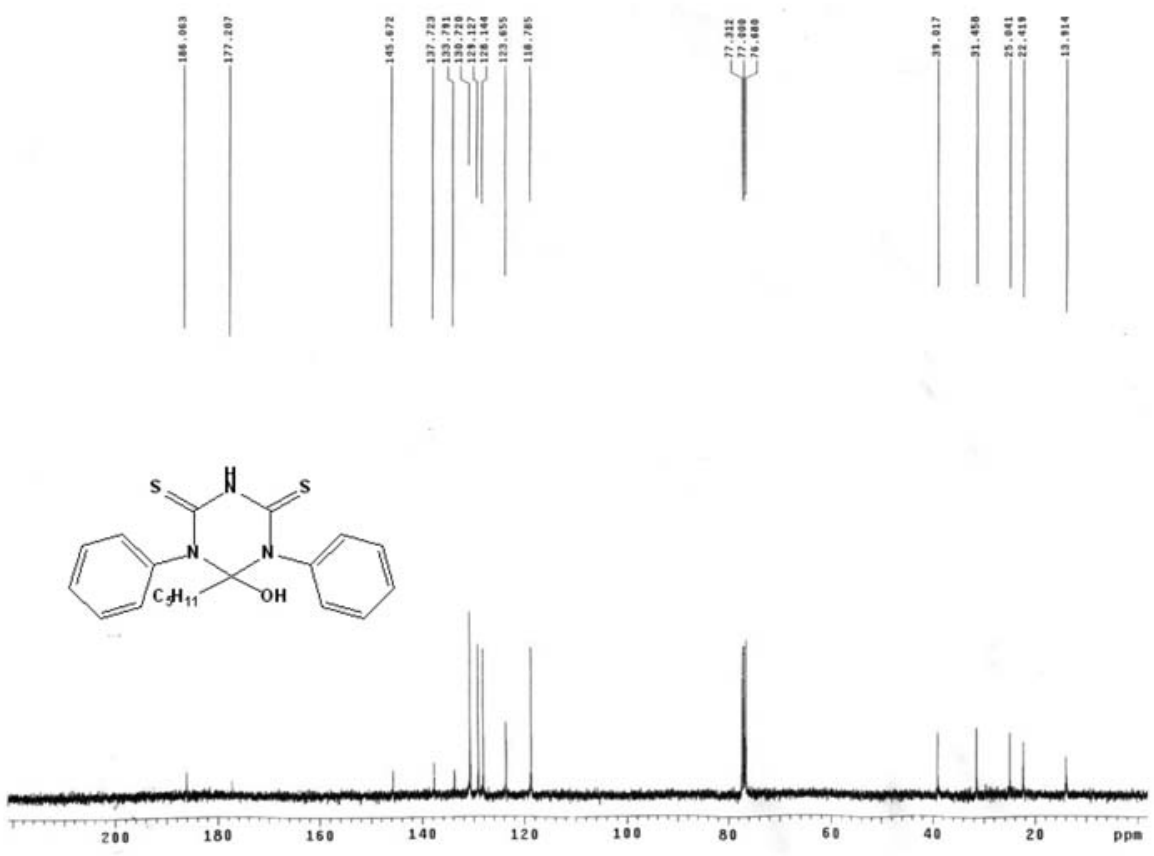

Figure S15. ${ }^{13} \mathrm{C}$ NMR $\left(\mathrm{CDCl}_{3}\right)$ spectrum of 6-hydroxy-6-pentyl-1,5-diphenyl-1,3,5-triazinane-2,4-dithione (5).

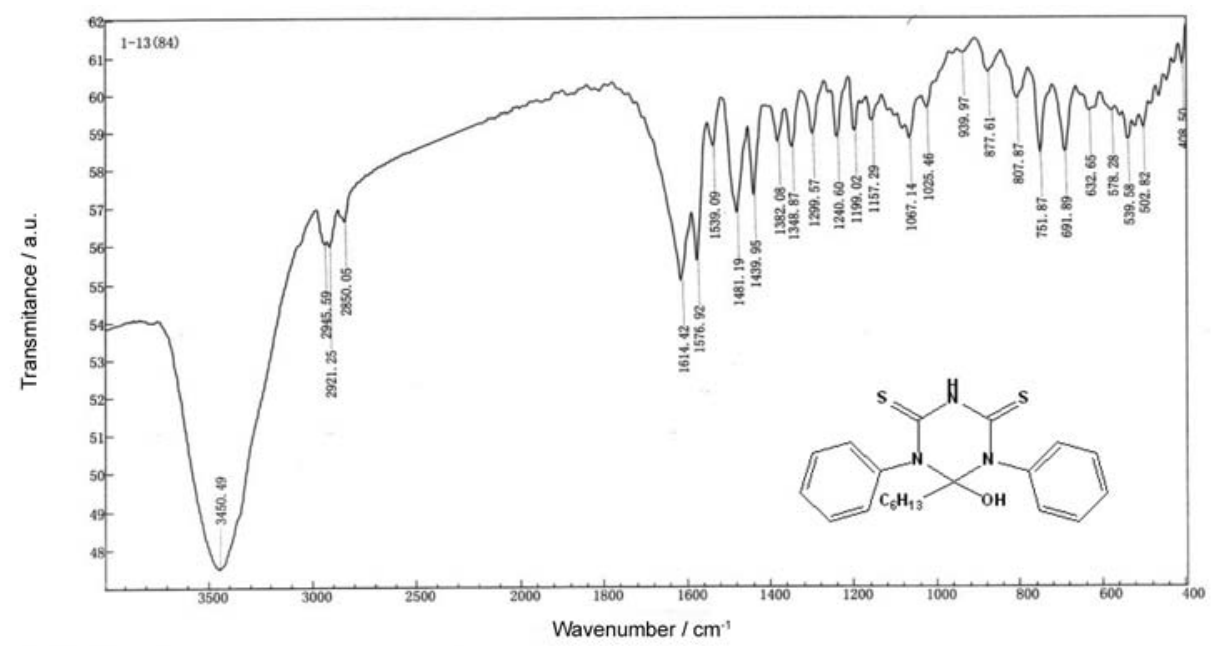

Figure S16. IR (KBr) spectrum of 6-hydroxy-6-hexyl-1,5-diphenyl-1,3,5-triazinane-2,4-dithione (6). 

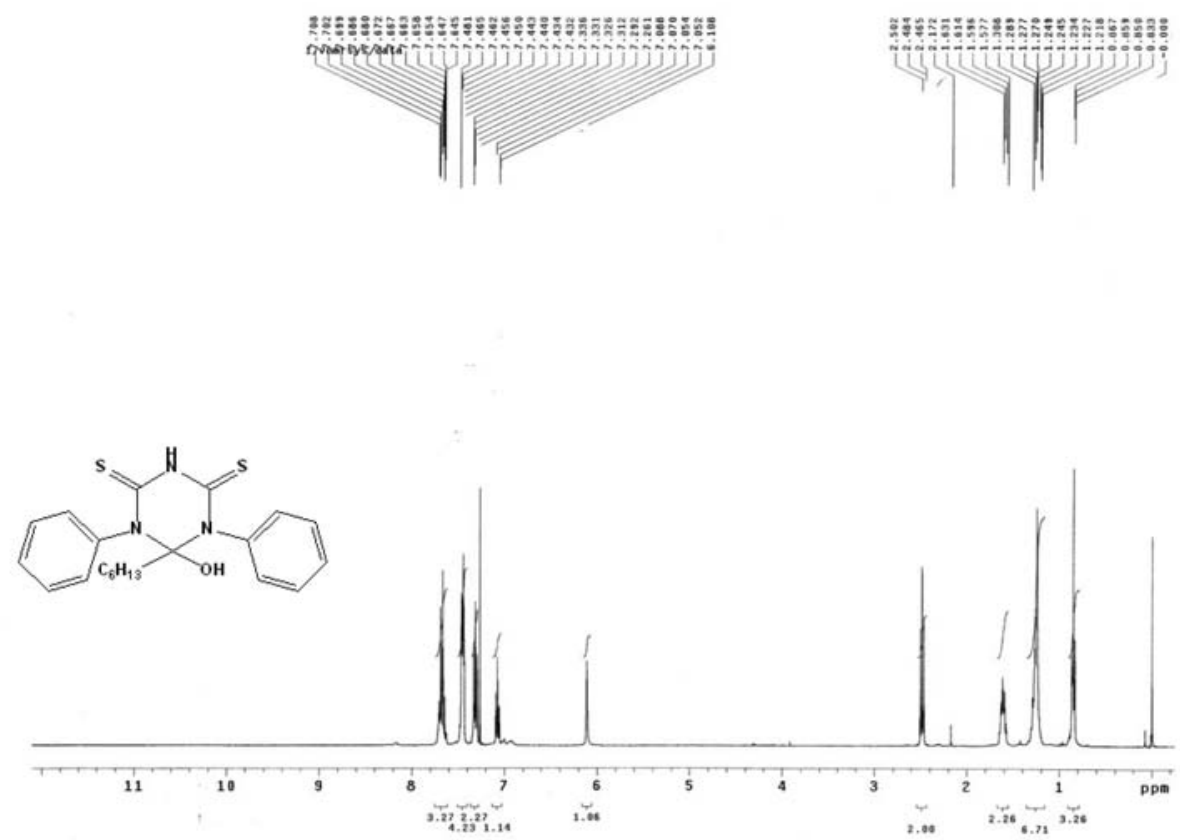

Figure S17. ${ }^{1} \mathrm{H}$ NMR $\left(\mathrm{CDCl}_{3}\right)$ spectrum of 6-hydroxy-6-hexyl-1,5-diphenyl-1,3,5-triazinane-2,4-dithione (6).
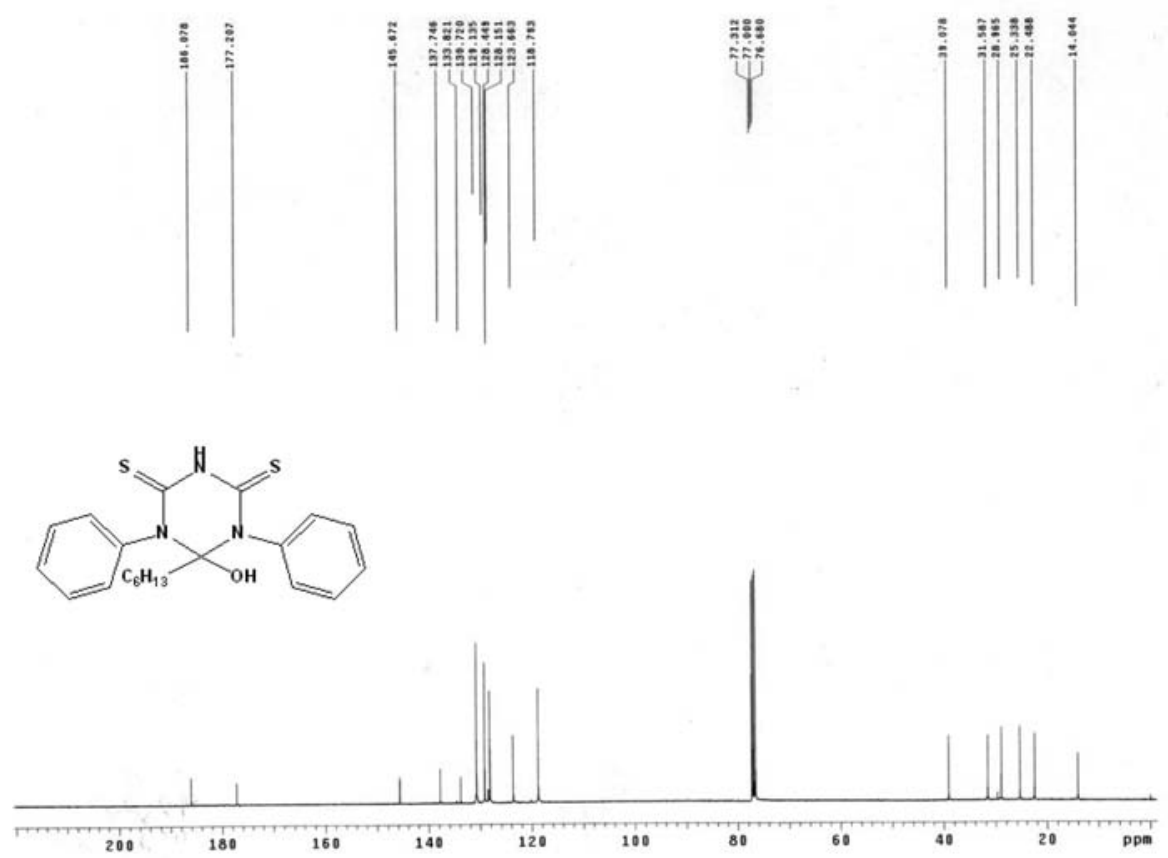

Figure S18. ${ }^{13} \mathrm{C} \mathrm{NMR}\left(\mathrm{CDCl}_{3}\right)$ spectrum of 6-hydroxy-6-hexyl-1,5-diphenyl-1,3,5-triazinane-2,4-dithione (6). 


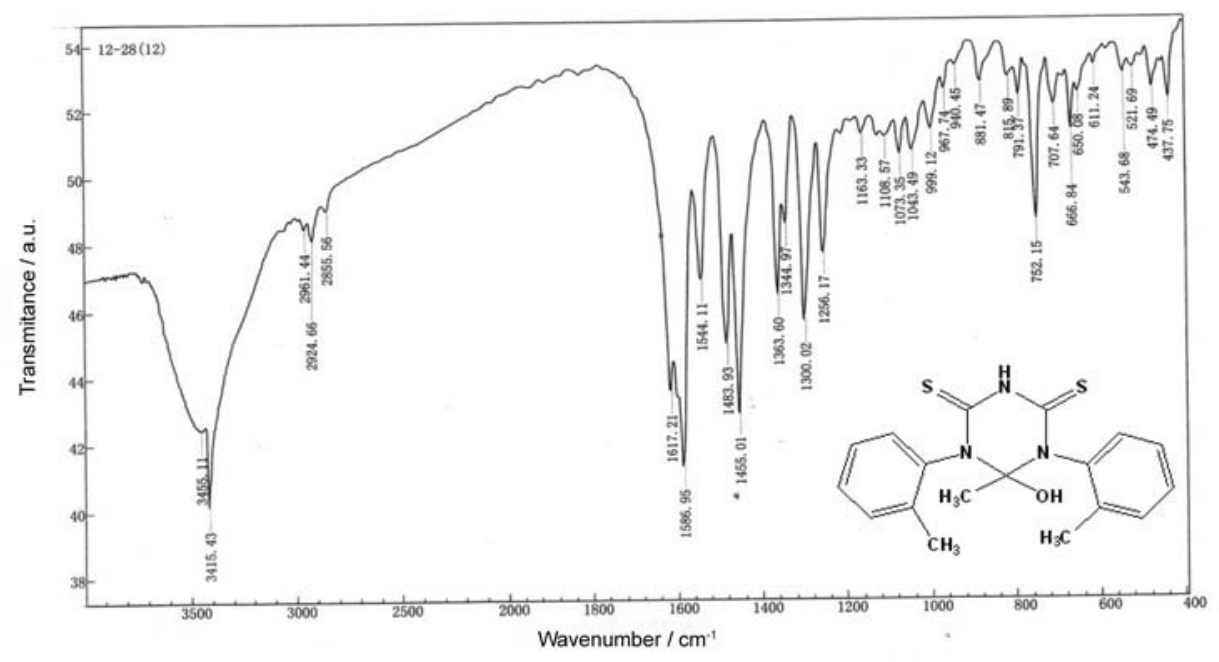

Figure S19. IR (KBr) spectrum of 6-hydroxy-6-methyl-1,5-di(2-methylphenyl)-1,3,5-triazinane-2,4-dithione (7).

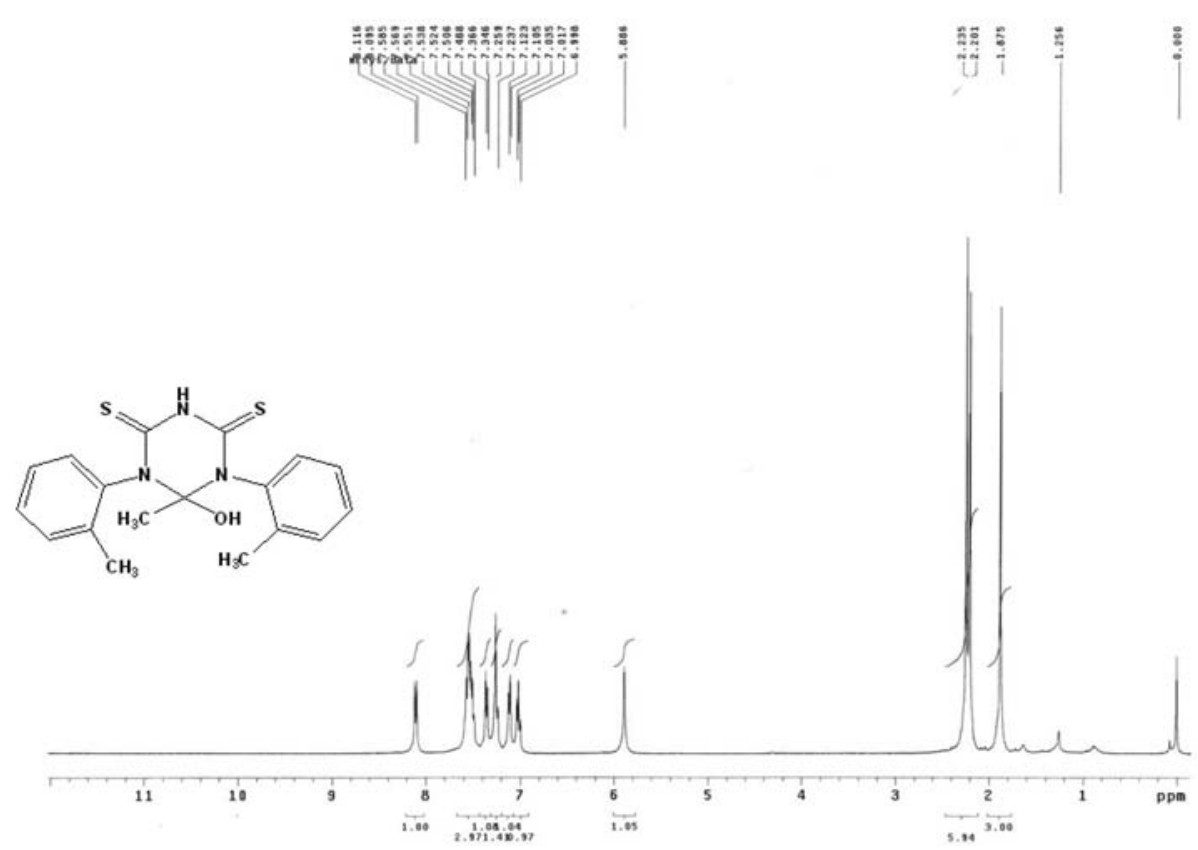

Figure S20. ${ }^{1} \mathrm{H}$ NMR $\left(\mathrm{CDCl}_{3}\right)$ spectrum of 6-hydroxy-6-methyl-1,5-di(2-methylphenyl)-1,3,5-triazinane-2,4-dithione (7). 

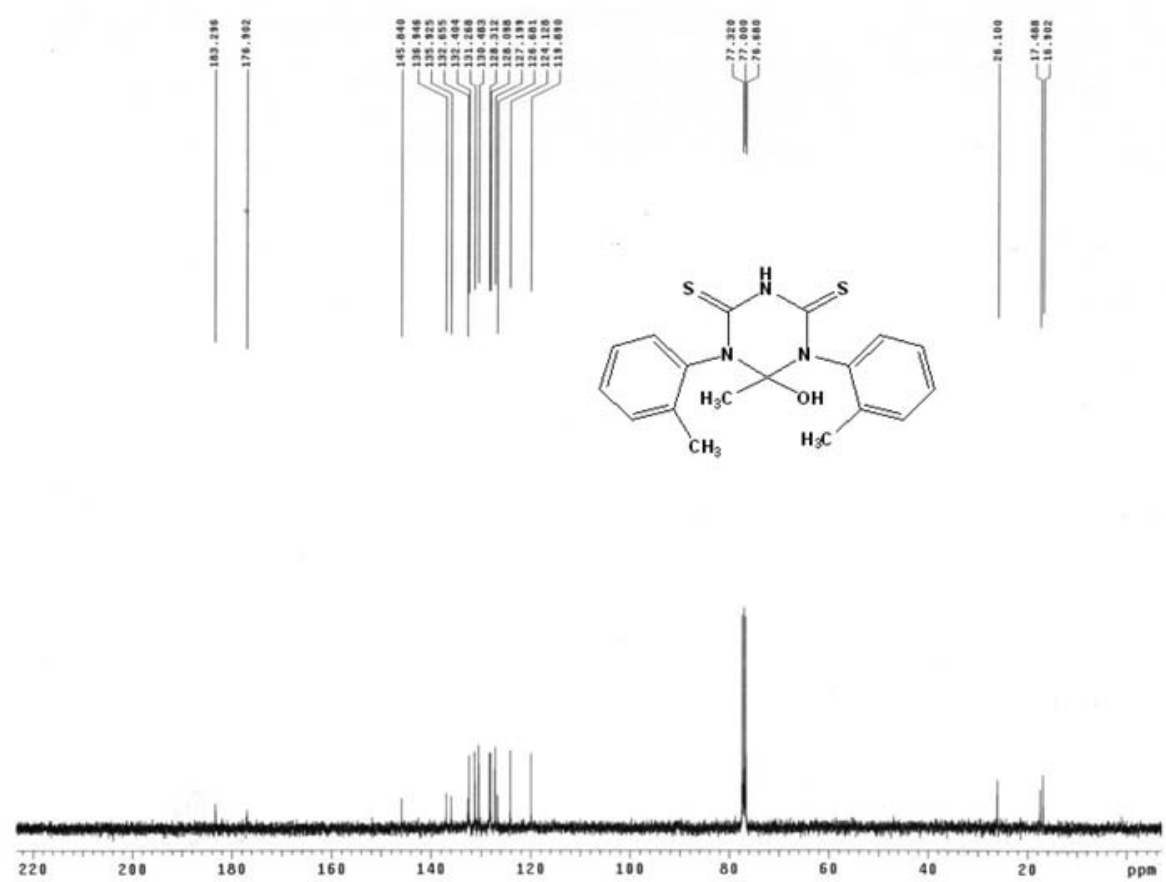

Figure S21. ${ }^{13} \mathrm{C}$ NMR $\left(\mathrm{CDCl}_{3}\right)$ spectrum of 6-hydroxy-6-methyl-1,5-di(2-methylphenyl)-1,3,5-triazinane-2,4-dithione (7).

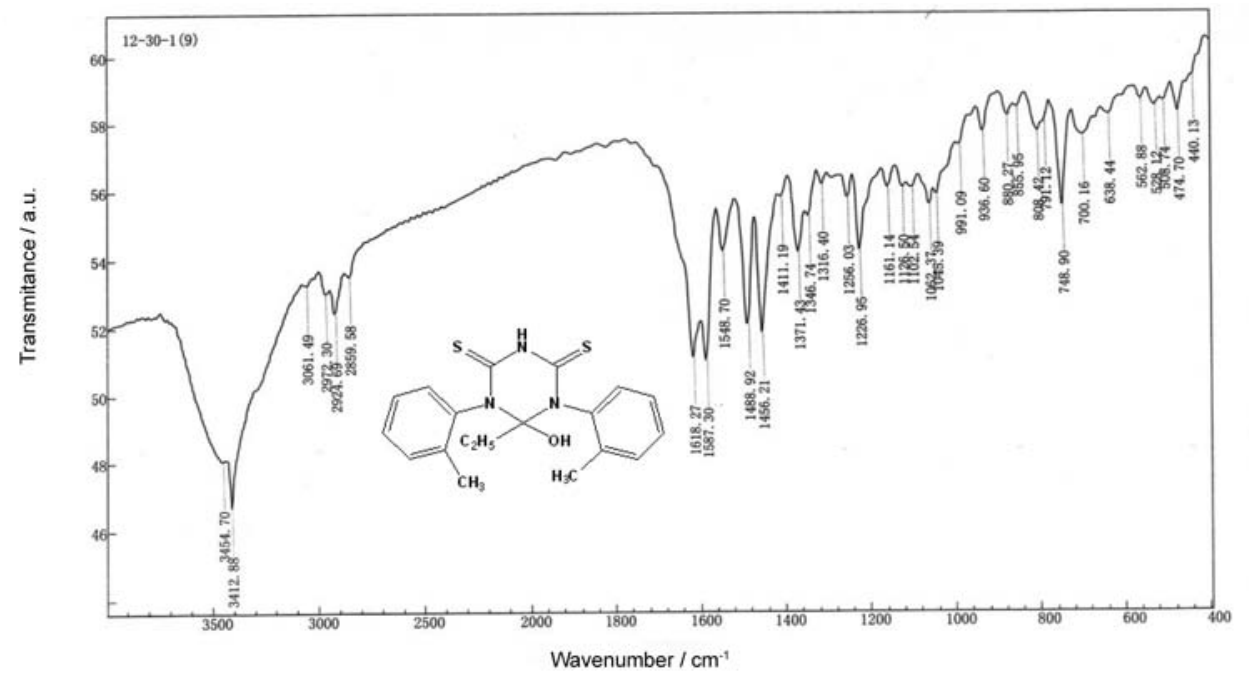

Figure S22. IR (KBr) spectrum of 6-hydroxy-6-ethyl-1,5-di(2-methylphenyl)-1,3,5-triazinane-2,4-dithione (8). 


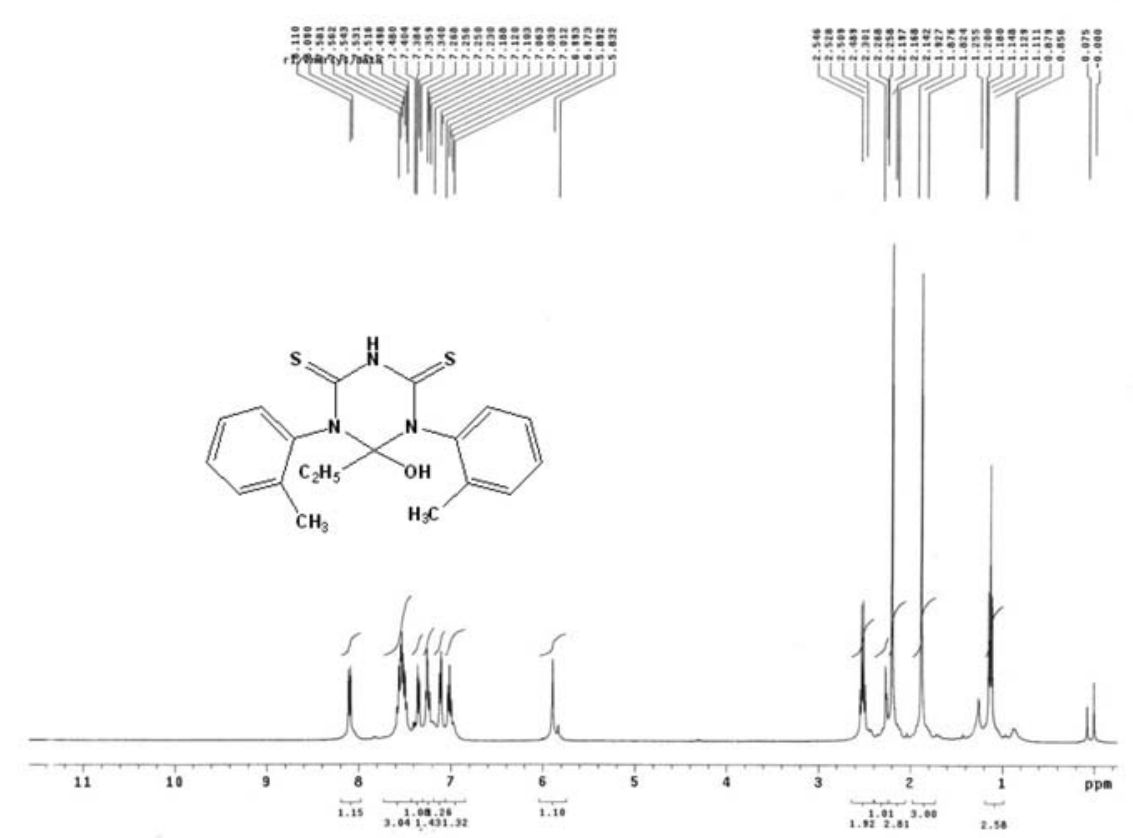

Figure S23. ${ }^{1} \mathrm{H}$ NMR $\left(\mathrm{CDCl}_{3}\right)$ spectrum of 6-hydroxy-6-ethyl-1,5-di(2-methylphenyl)-1,3,5-triazinane-2,4-dithione (8).
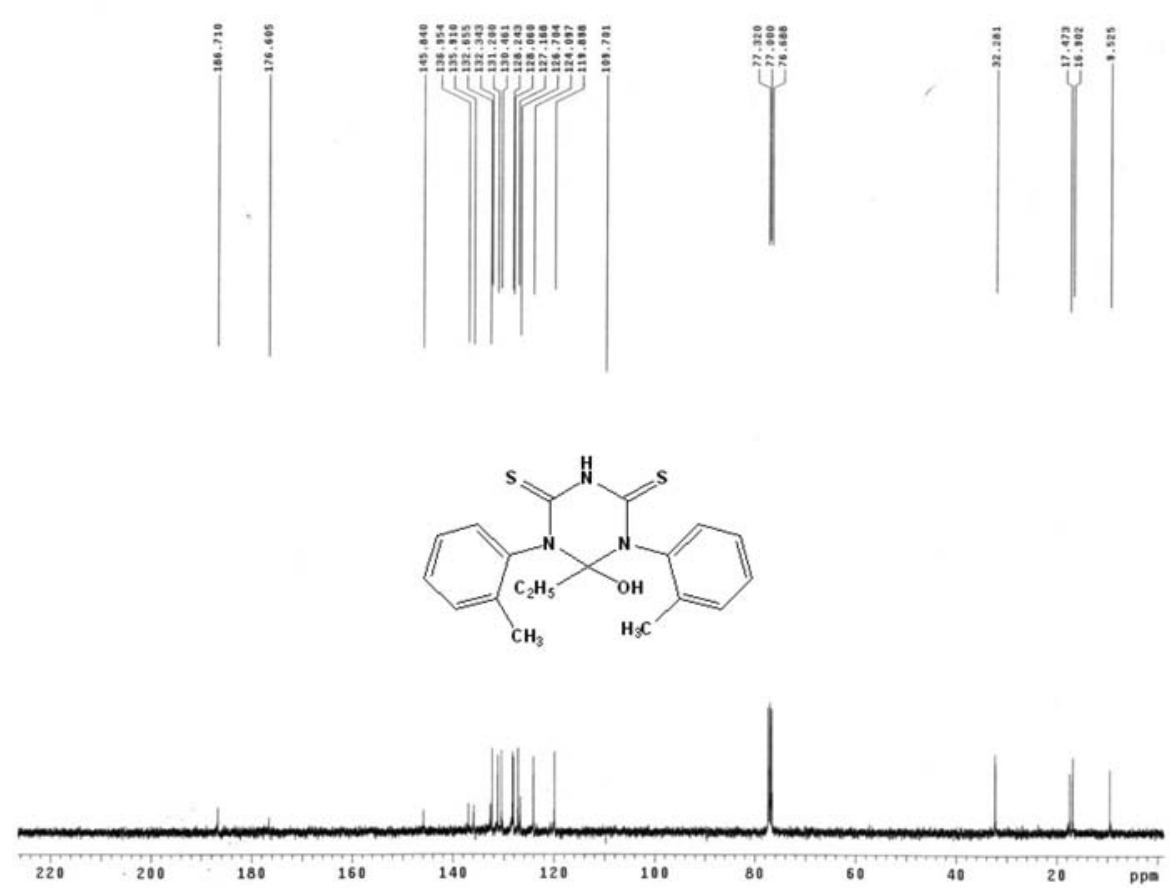

Figure S24. ${ }^{13} \mathrm{C}$ NMR $\left(\mathrm{CDCl}_{3}\right)$ spectrum of 6-hydroxy-6-ethyl-1,5-di(2-methylphenyl)-1,3,5-triazinane-2,4-dithione $(\mathbf{8})$. 


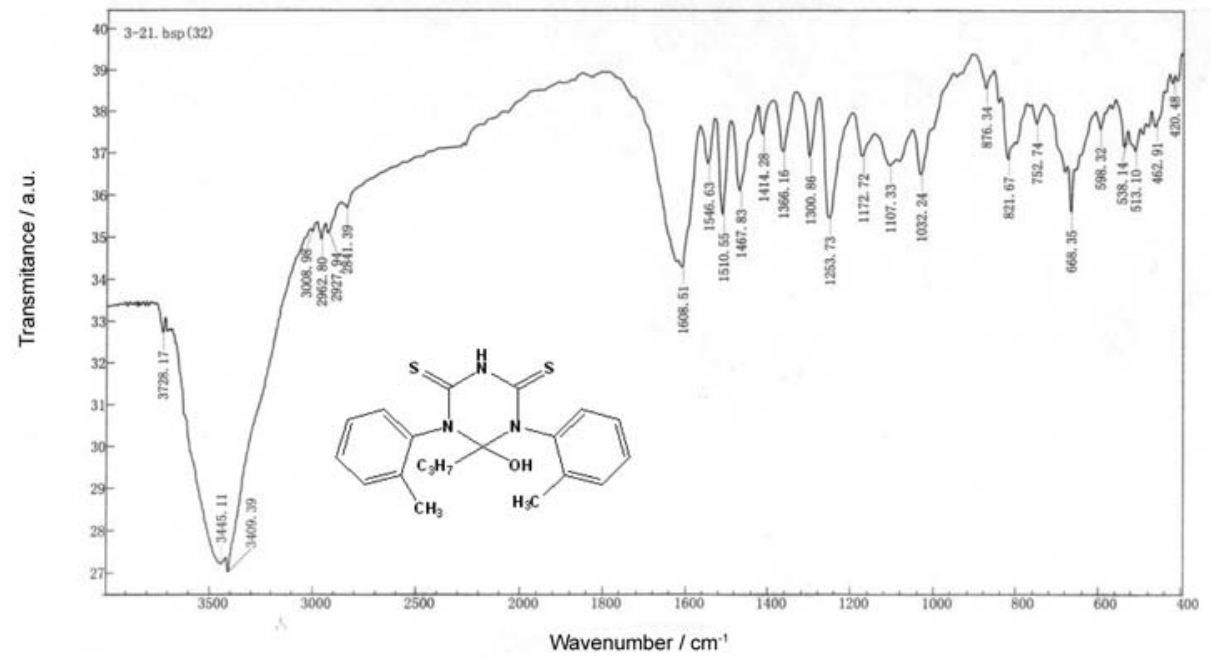

Figure S25. IR (KBr) spectrum of 6-hydroxy-6-propyl-1,5-di(2-methylphenyl)-1,3,5-triazinane-2,4-dithione (9).

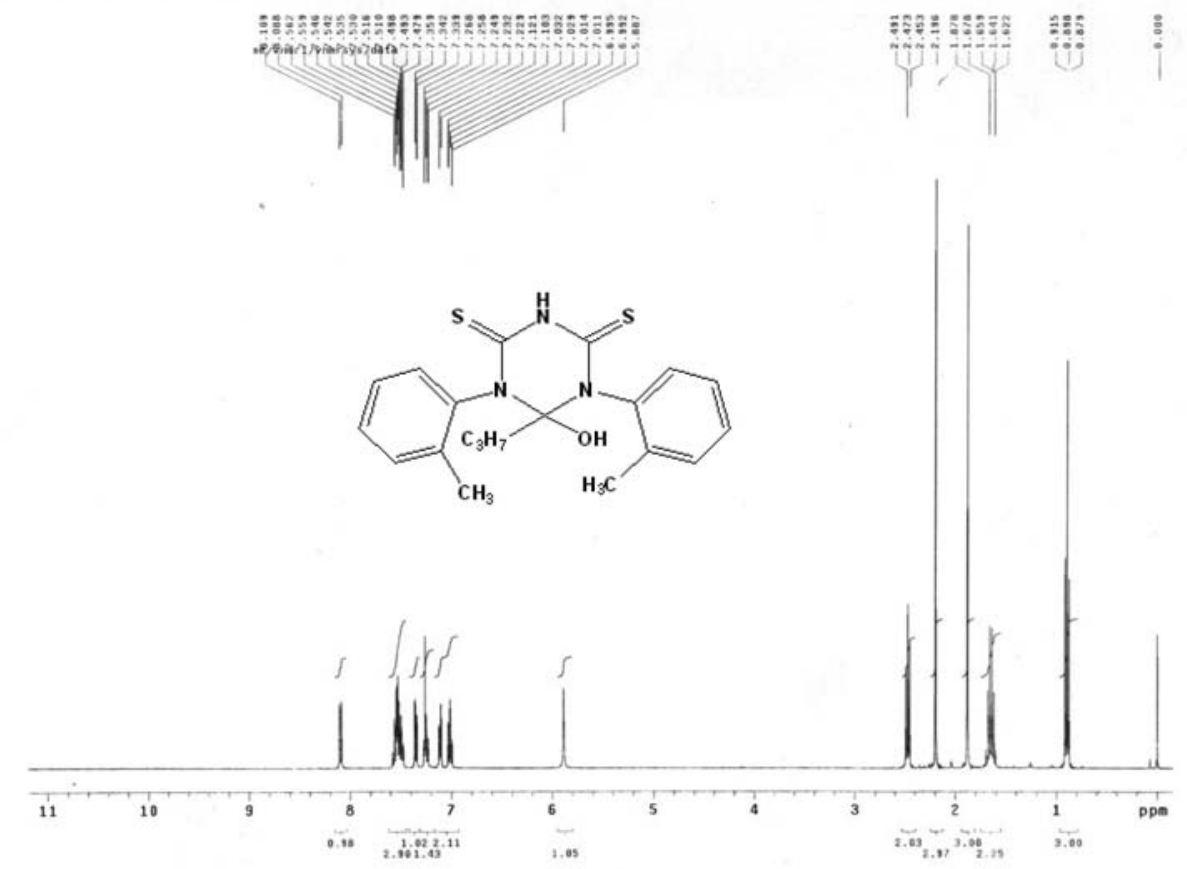

Figure S26. ${ }^{1} \mathrm{H}$ NMR $\left(\mathrm{CDCl}_{3}\right)$ spectrum of 6-hydroxy-6-propyl-1,5-di(2-methylphenyl)-1,3,5-triazinane-2,4-dithione (9). 

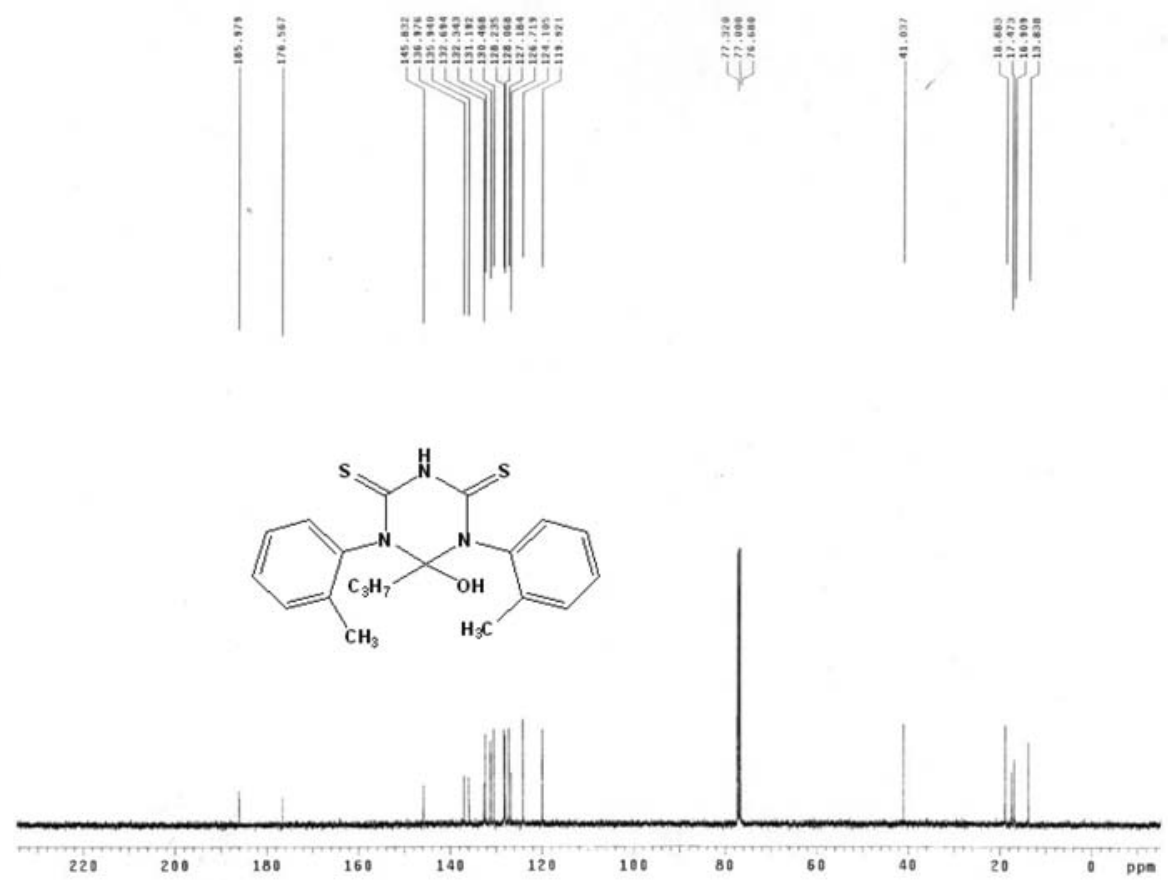

Figure S27. ${ }^{13} \mathrm{C}$ NMR $\left(\mathrm{CDCl}_{3}\right)$ spectrum of 6-hydroxy-6-propyl-1,5-di(2-methylphenyl)-1,3,5-triazinane-2,4-dithione (9).

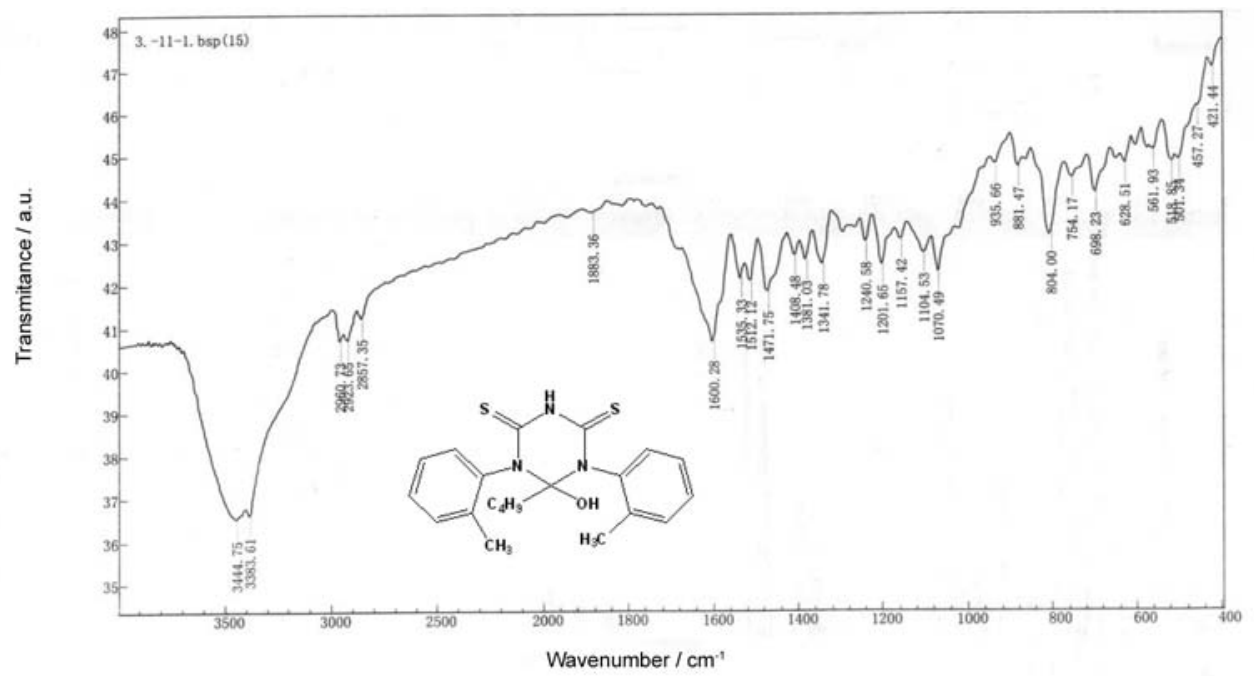

Figure S28. IR (KBr) spectrum of 6-hydroxy-6-butyl-1,5-di(2-methylphenyl)-1,3,5-triazinane-2,4-dithione (10). 


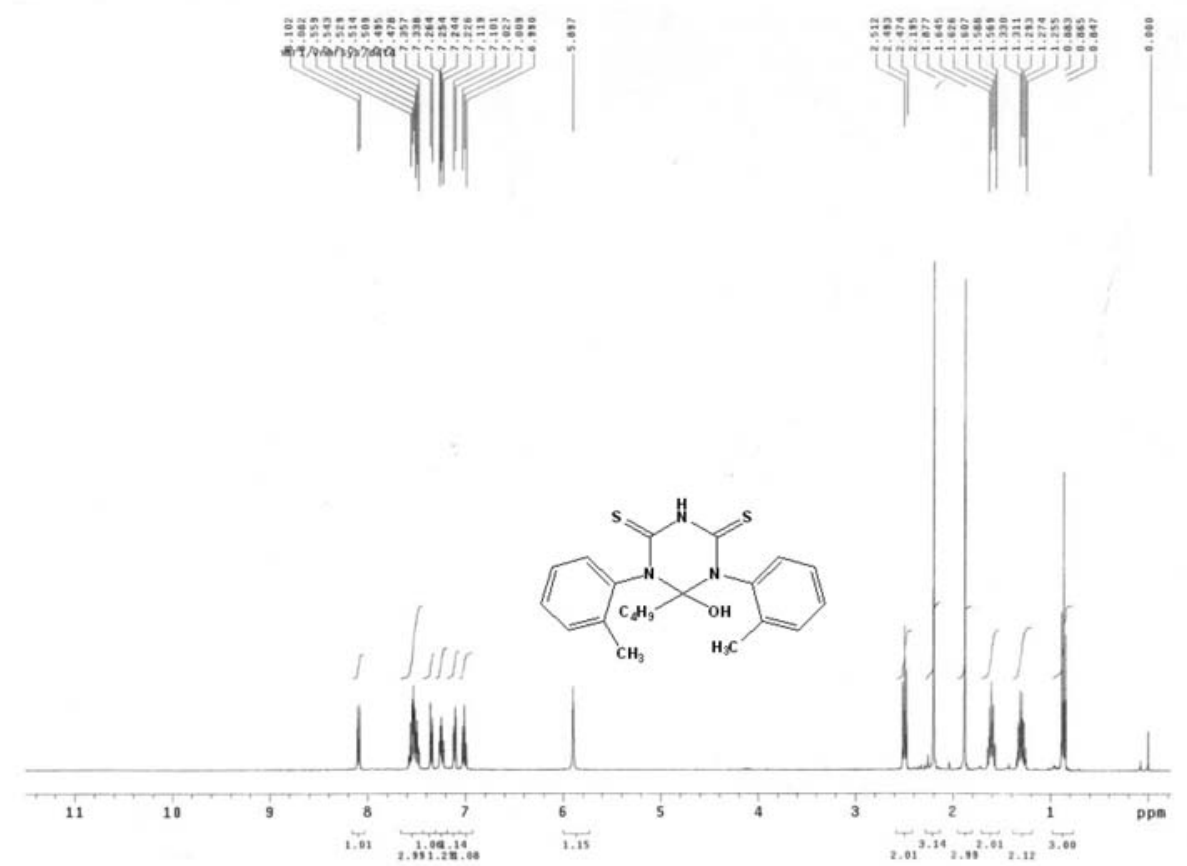

Figure S29. ${ }^{1} \mathrm{H}$ NMR $\left(\mathrm{CDCl}_{3}\right)$ spectrum of 6-hydroxy-6-butyl-1,5-di(2-methylphenyl)-1,3,5-triazinane-2,4-dithione (10).
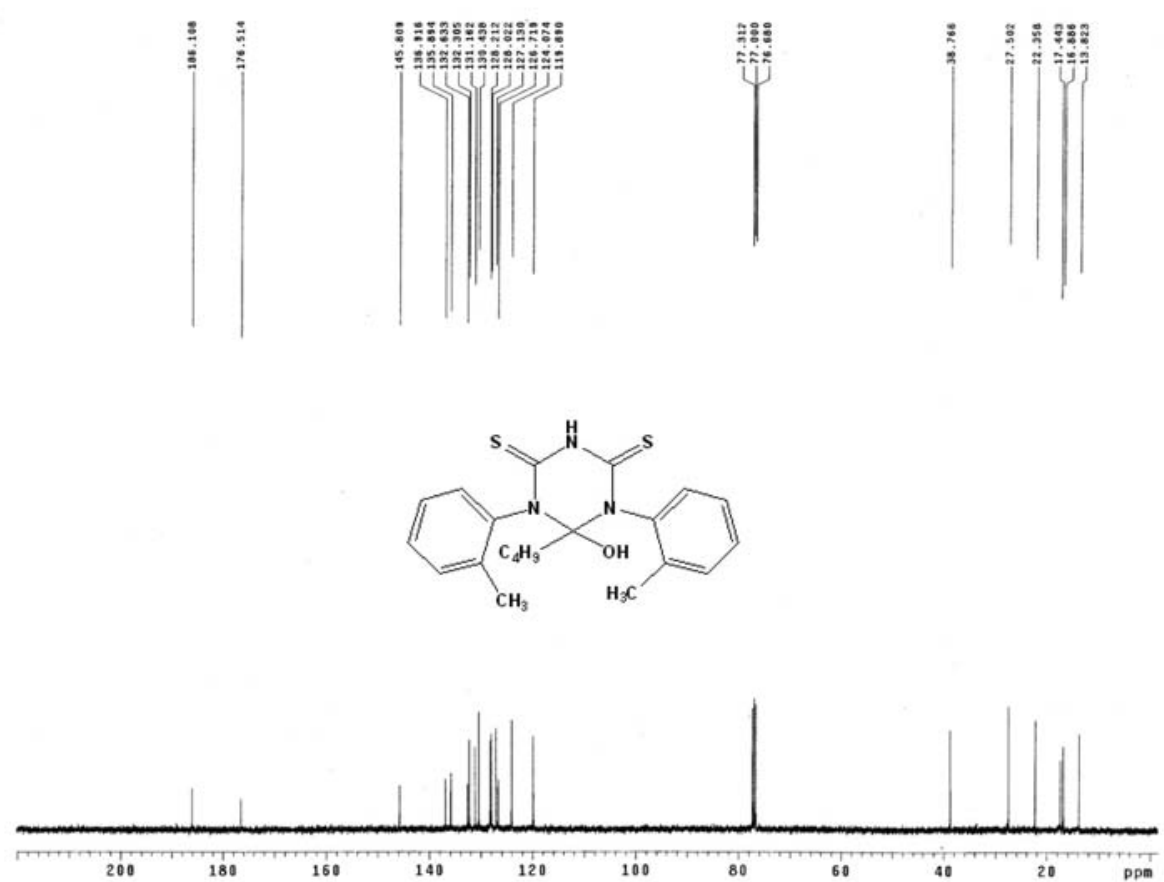

Figure S30. ${ }^{13} \mathrm{C} \mathrm{NMR}\left(\mathrm{CDCl}_{3}\right)$ spectrum of 6-hydroxy-6-butyl-1,5-di(2-methylphenyl)-1,3,5-triazinane-2,4-dithione (10). 


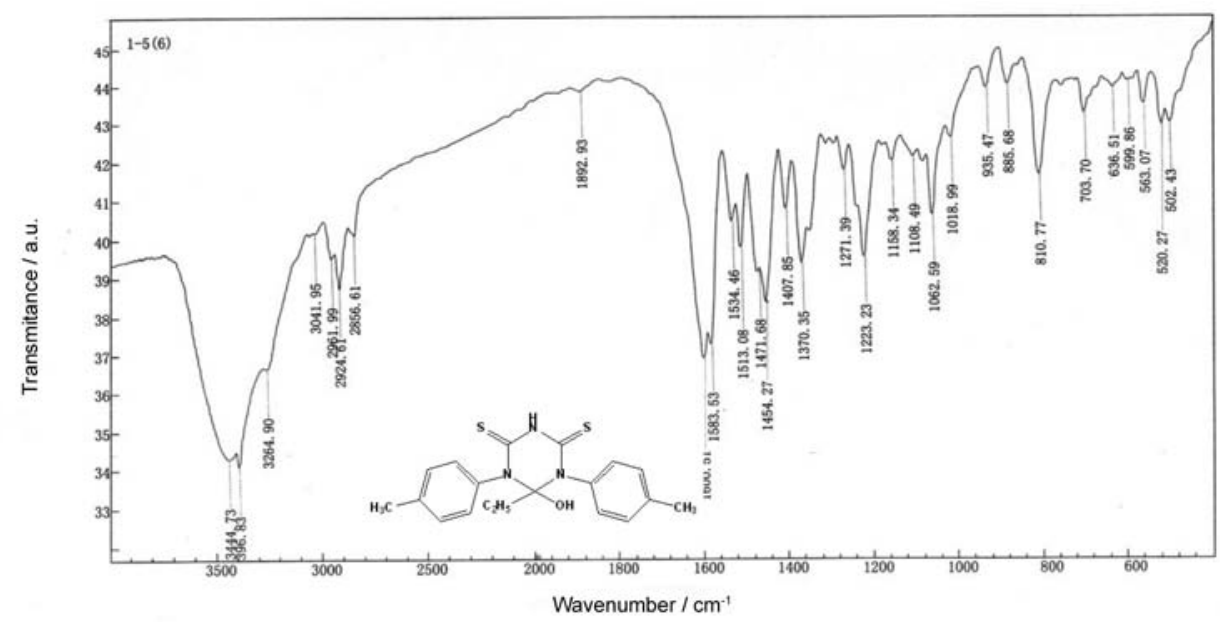

Figure S31. IR (KBr) spectrum of 6-hydroxy-6-ethyl-1,5-di(4-methylphenyl)-1,3,5-triazinane-2,4-dithione (11).

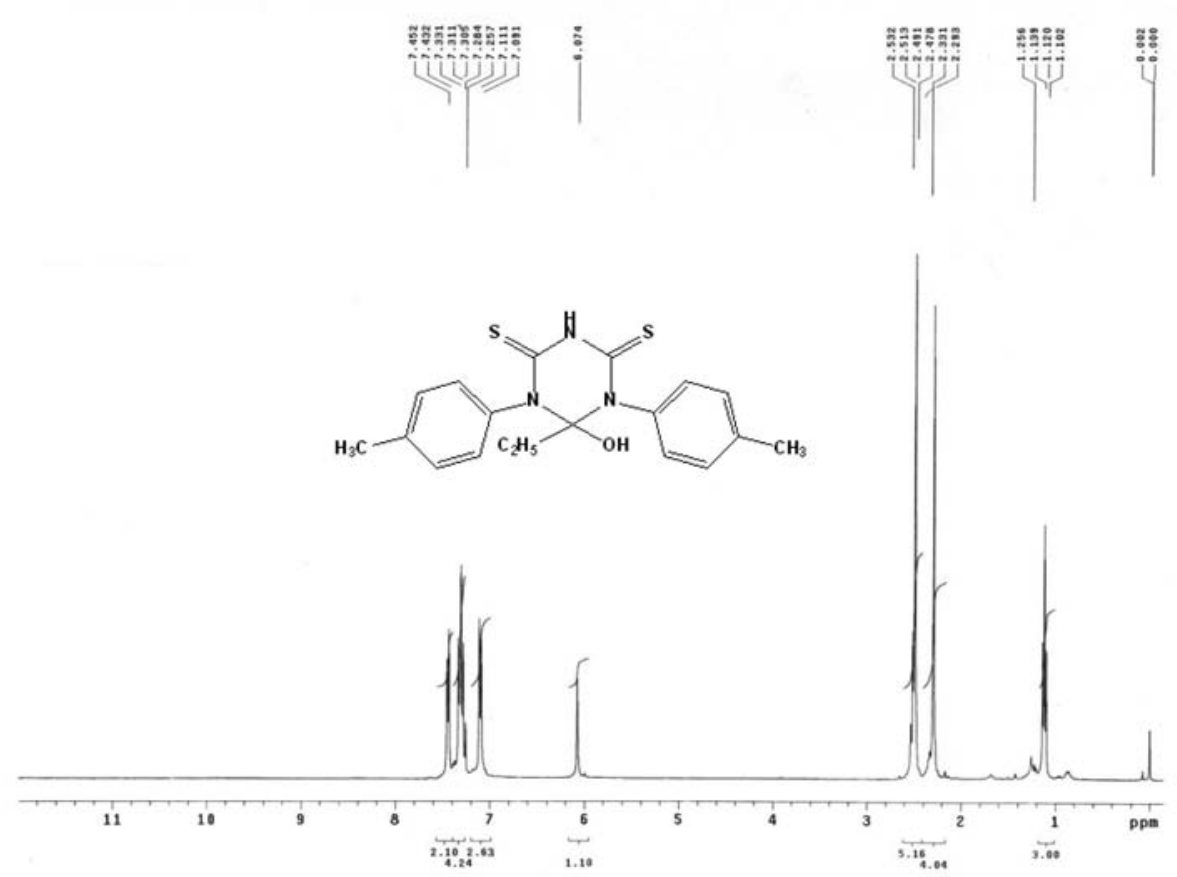

Figure S32. ${ }^{1} \mathrm{H}$ NMR $\left(\mathrm{CDCl}_{3}\right)$ spectrum of 6-hydroxy-6-ethyl-1,5-di(4-methylphenyl)-1,3,5-triazinane-2,4-dithione (11). 

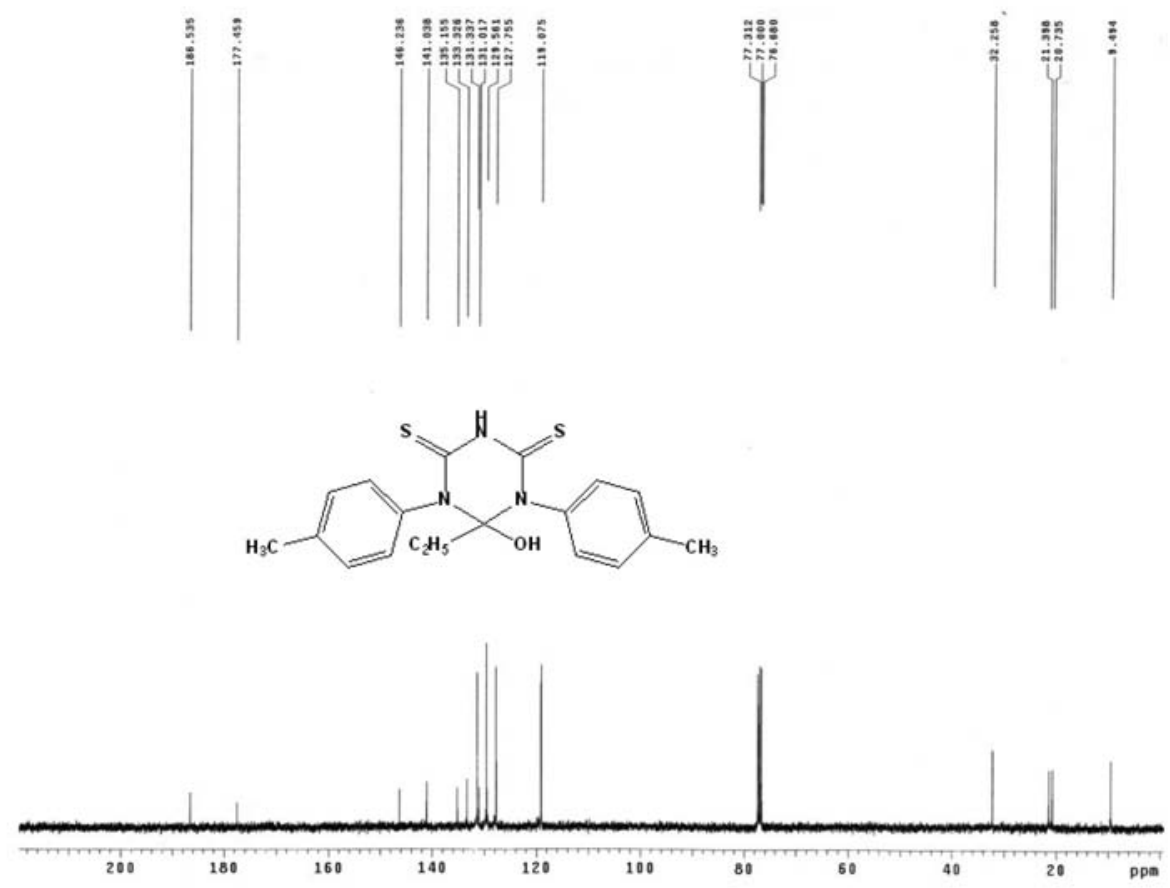

Figure S33. ${ }^{13} \mathrm{C}$ NMR $\left(\mathrm{CDCl}_{3}\right)$ spectrum of 6-hydroxy-6-ethyl-1,5-di(4-methylphenyl)-1,3,5-triazinane-2,4-dithione (11).

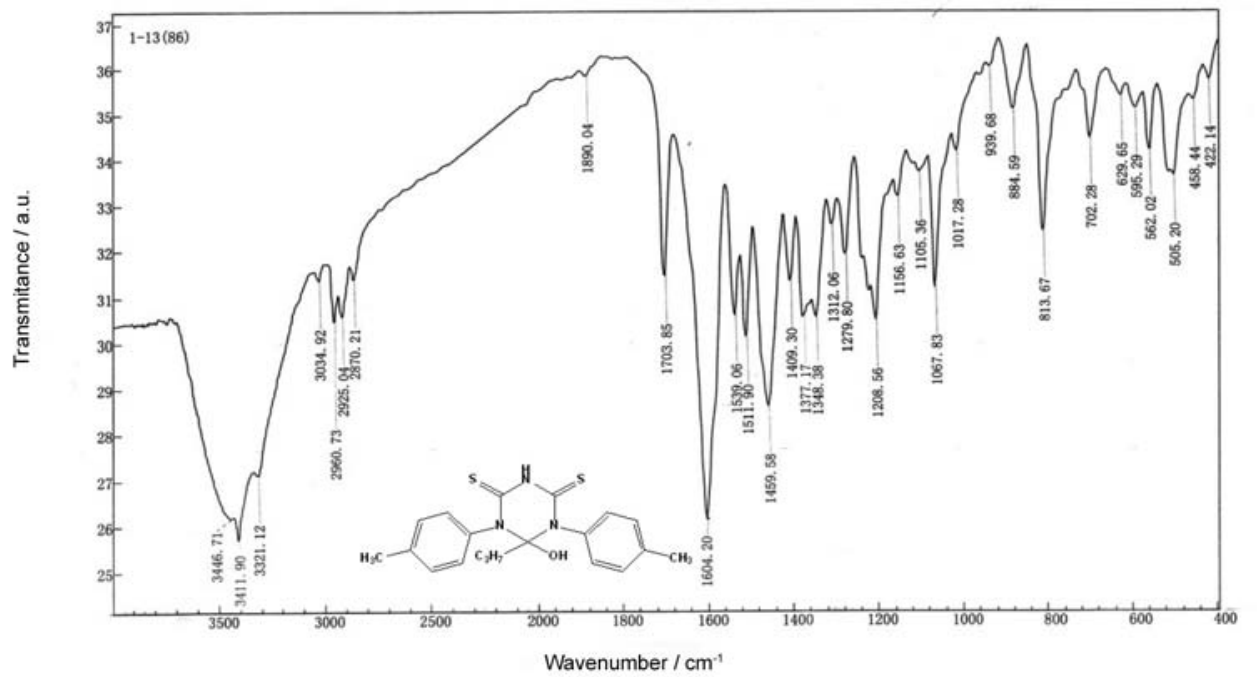

Figure S34. IR (KBr) spectrum of 6-hydroxy-6-propyl-1,5-di(4-methylphenyl)-1,3,5-triazinane-2,4-dithione (12). 


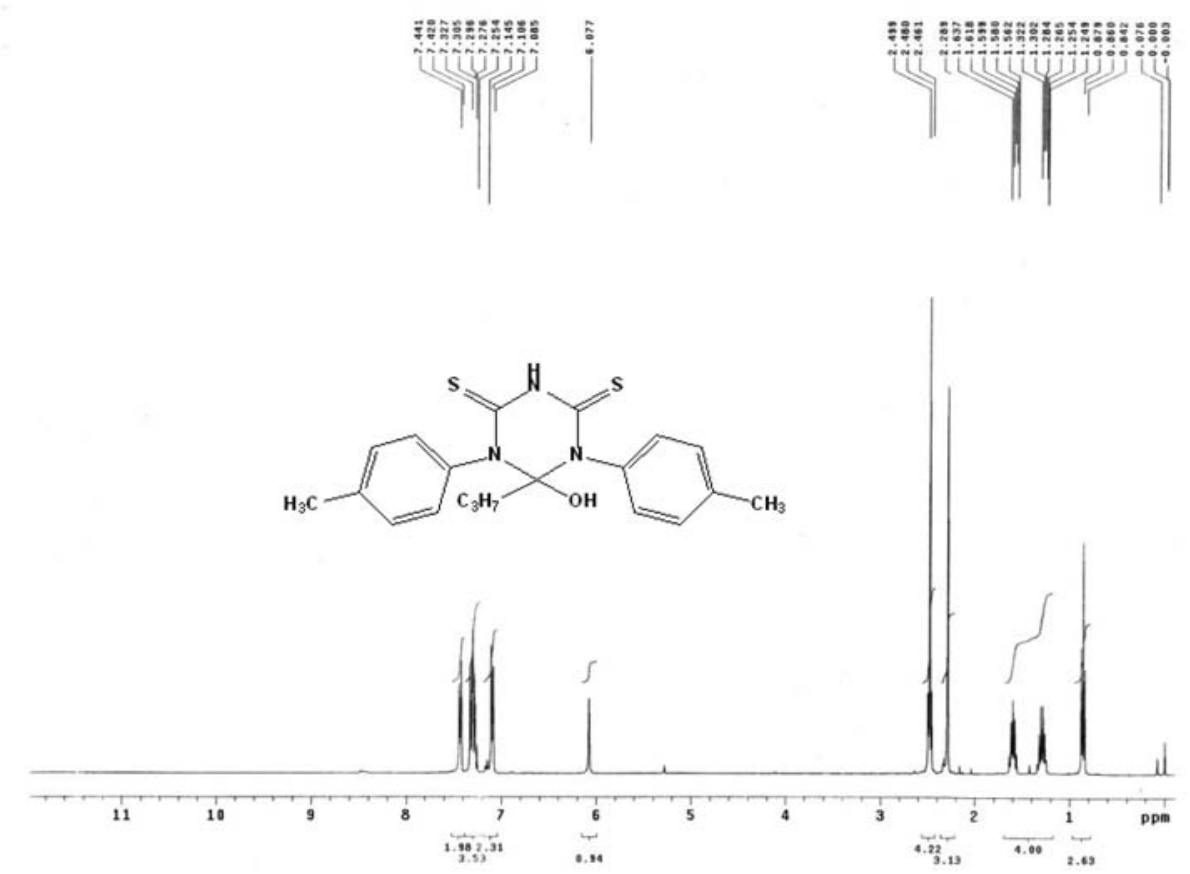

Figure S35. ${ }^{1} \mathrm{H}$ NMR $\left(\mathrm{CDCl}_{3}\right)$ spectrum of 6-hydroxy-6-propyl-1,5-di(4-methylphenyl)-1,3,5-triazinane-2,4-dithione (12).
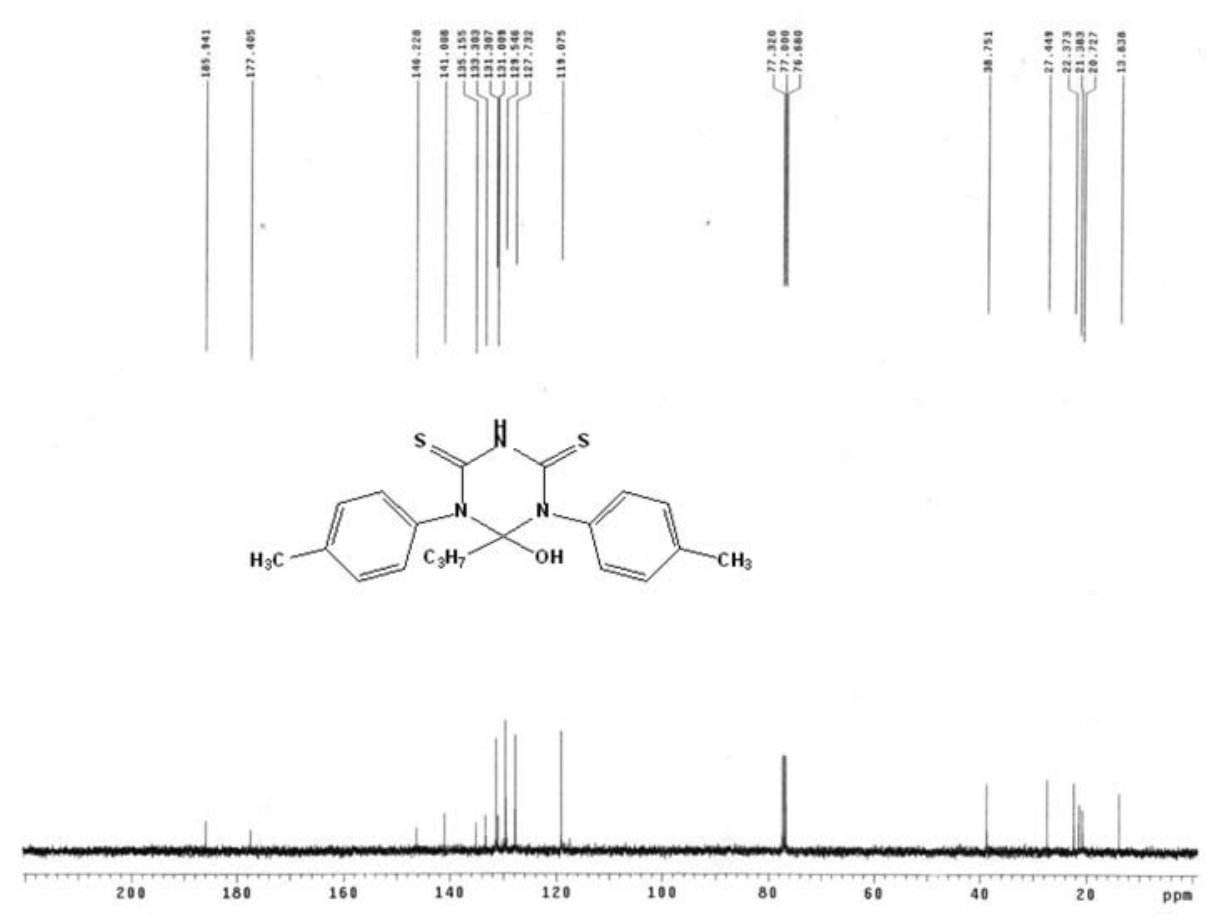

Figure S36. ${ }^{13} \mathrm{C} \mathrm{NMR}\left(\mathrm{CDCl}_{3}\right)$ spectrum of 6-hydroxy-6-propyl-1,5-di(4-methylphenyl)-1,3,5-triazinane-2,4-dithione (12). 


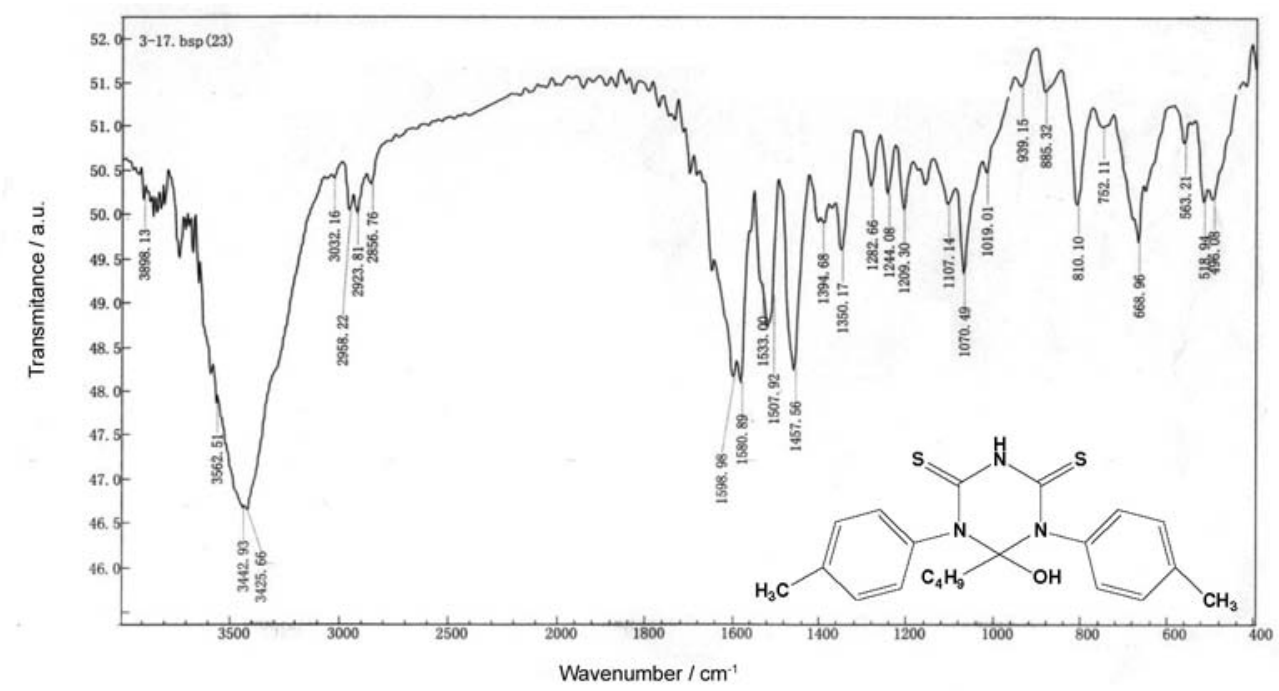

Figure S37. IR (KBr) spectrum of 6-hydroxy-6-butyl-1,5-di(4-methylphenyl)-1,3,5-triazinane-2,4-dithione (13).

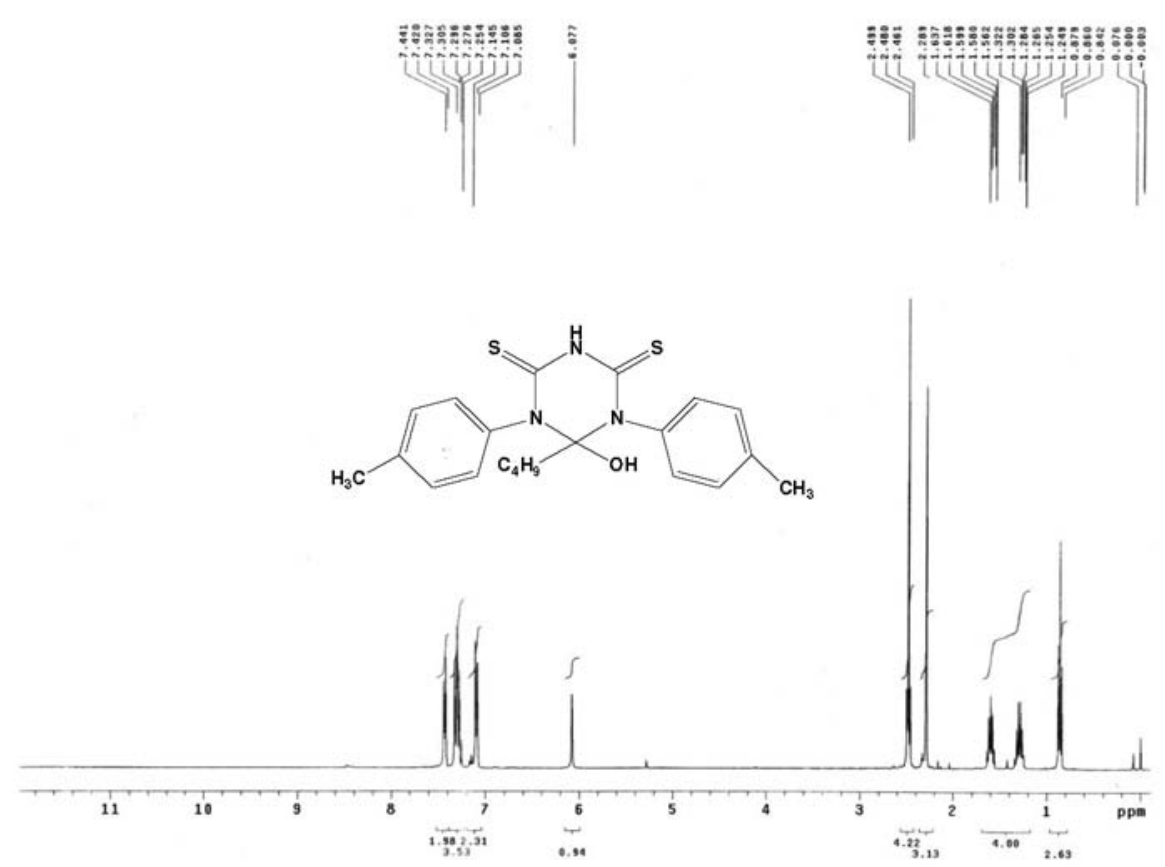

Figure S38. ${ }^{1} \mathrm{H}$ NMR $\left(\mathrm{CDCl}_{3}\right)$ spectrum of 6- ydroxyl-6-butyl-1,5-di(4-methylphenyl)-1,3,5-triazinane-2,4-dithione (13). 

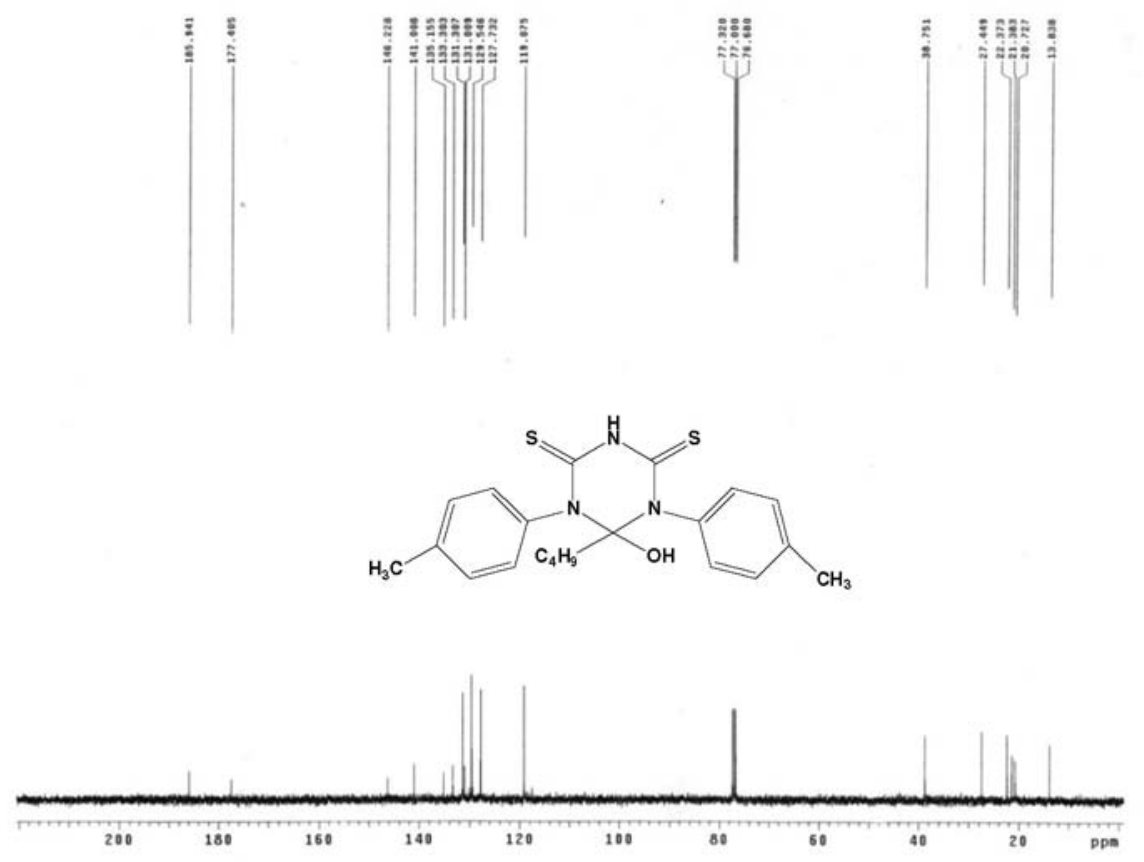

Figure S39. ${ }^{13} \mathrm{C}$ NMR $\left(\mathrm{CDCl}_{3}\right)$ spectrum of 6-hydroxy-6-butyl-1,5-di(4-methylphenyl)-1,3,5-triazinane-2,4-dithione (13).

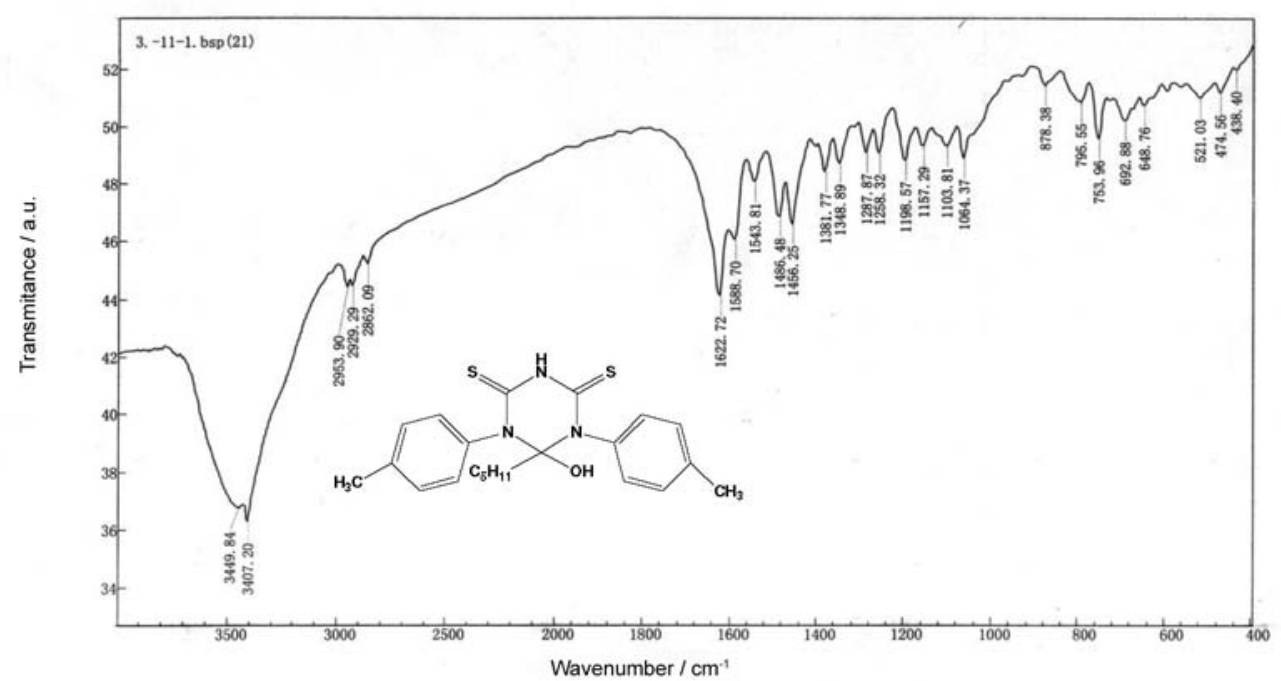

Figure S40. IR (KBr) spectrum of 6-hydroxy-6-pentyl-1,5-di(4-methylphenyl)-1,3,5-triazinane-2,4-dithione (14). 


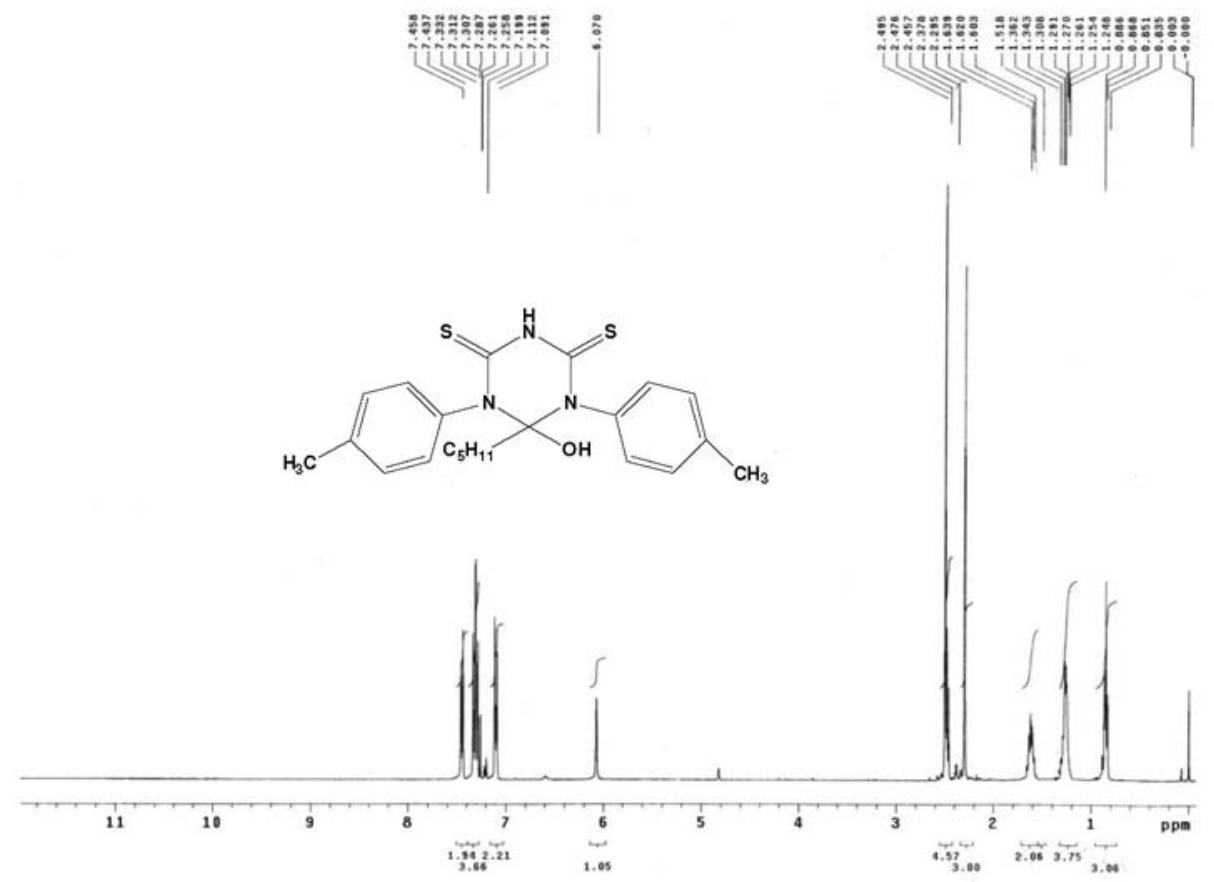

Figure S41. ${ }^{1} \mathrm{H}$ NMR $\left(\mathrm{CDCl}_{3}\right)$ spectrum of 6-hydroxy-6-pentyl-1,5-di(4-methylphenyl)-1,3,5-triazinane-2,4-dithione (14).
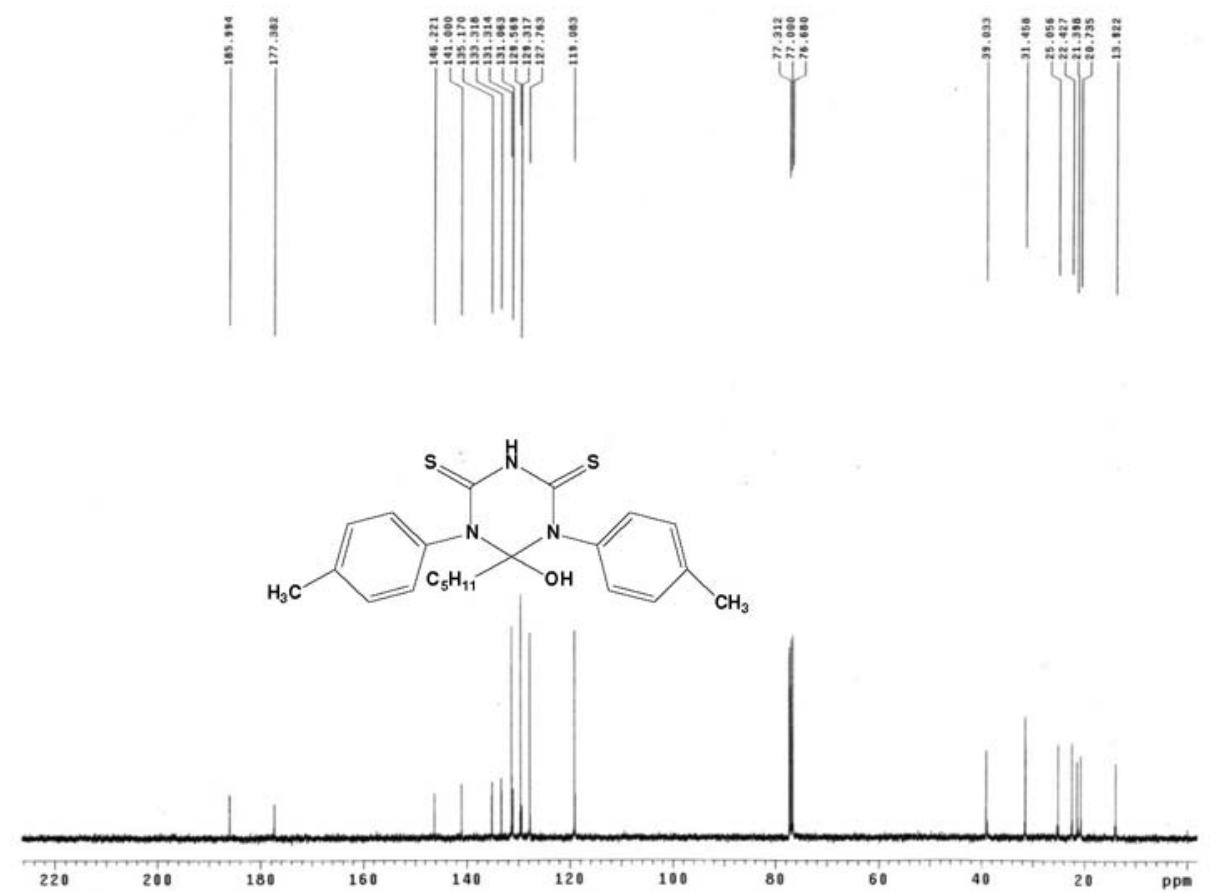

Figure S42. ${ }^{13} \mathrm{C}$ NMR $\left(\mathrm{CDCl}_{3}\right)$ spectrum of 6-hydroxy-6-pentyl-1,5-di(4-methylphenyl)-1,3,5-triazinane-2,4-dithione (14). 


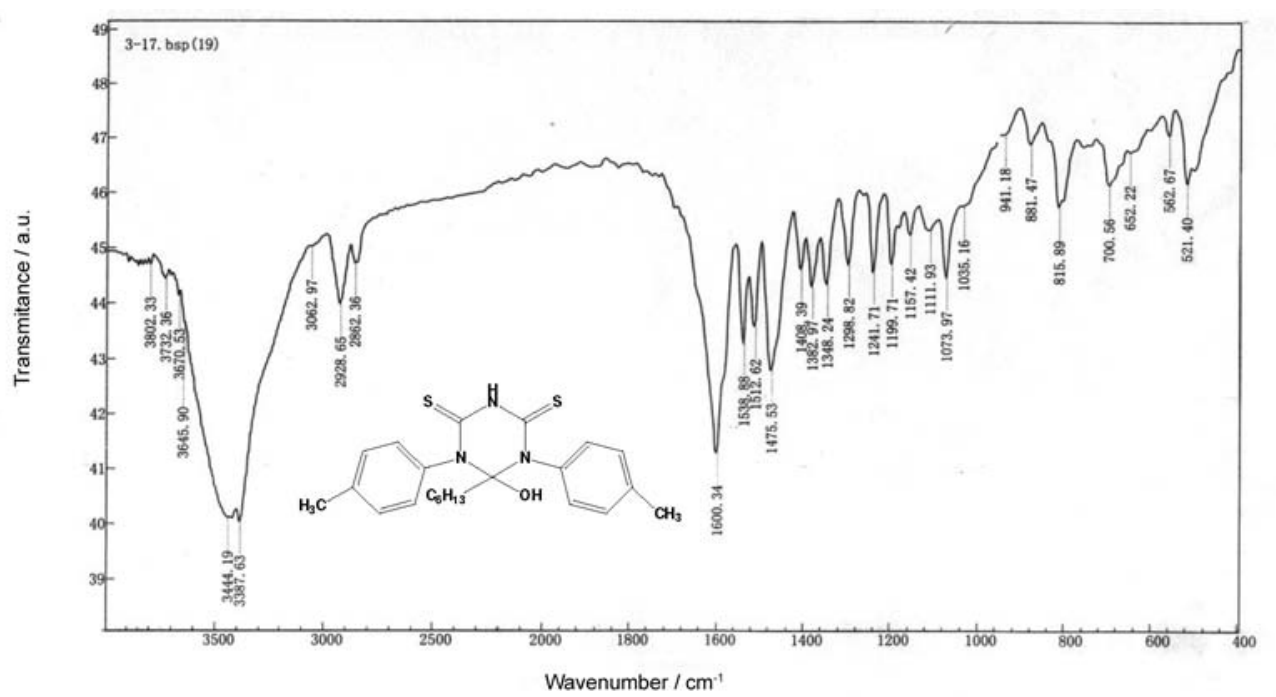

Figure S43. IR (KBr) spectrum of 6-hydroxy-6-hexyl-1,5-di(4-methylphenyl)-1,3,5-triazinane-2,4-dithione (15).

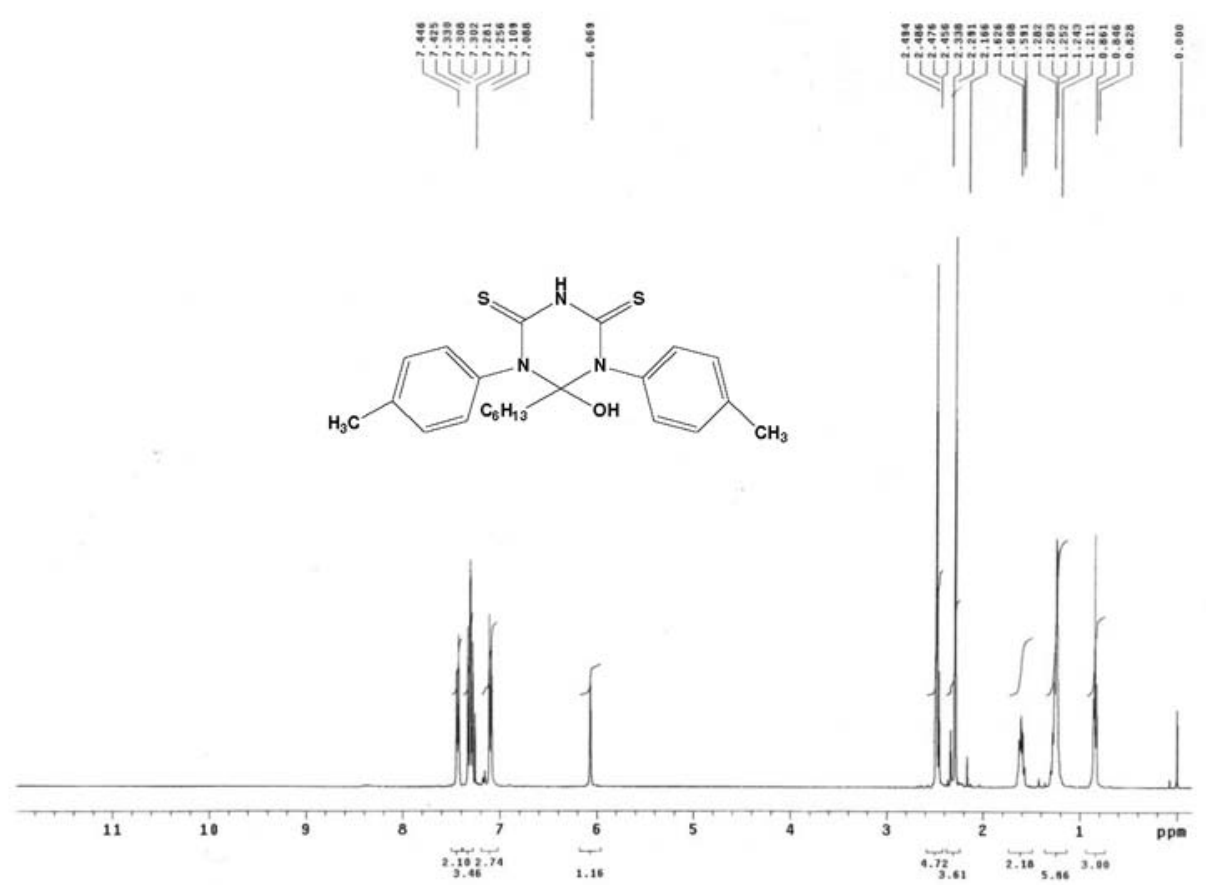

Figure S44. ${ }^{1} \mathrm{H}$ NMR $\left(\mathrm{CDCl}_{3}\right)$ spectrum of 6-hydroxy-6-hexyl-1,5-di(4-methylphenyl)-1,3,5-triazinane-2,4-dithione (15). 

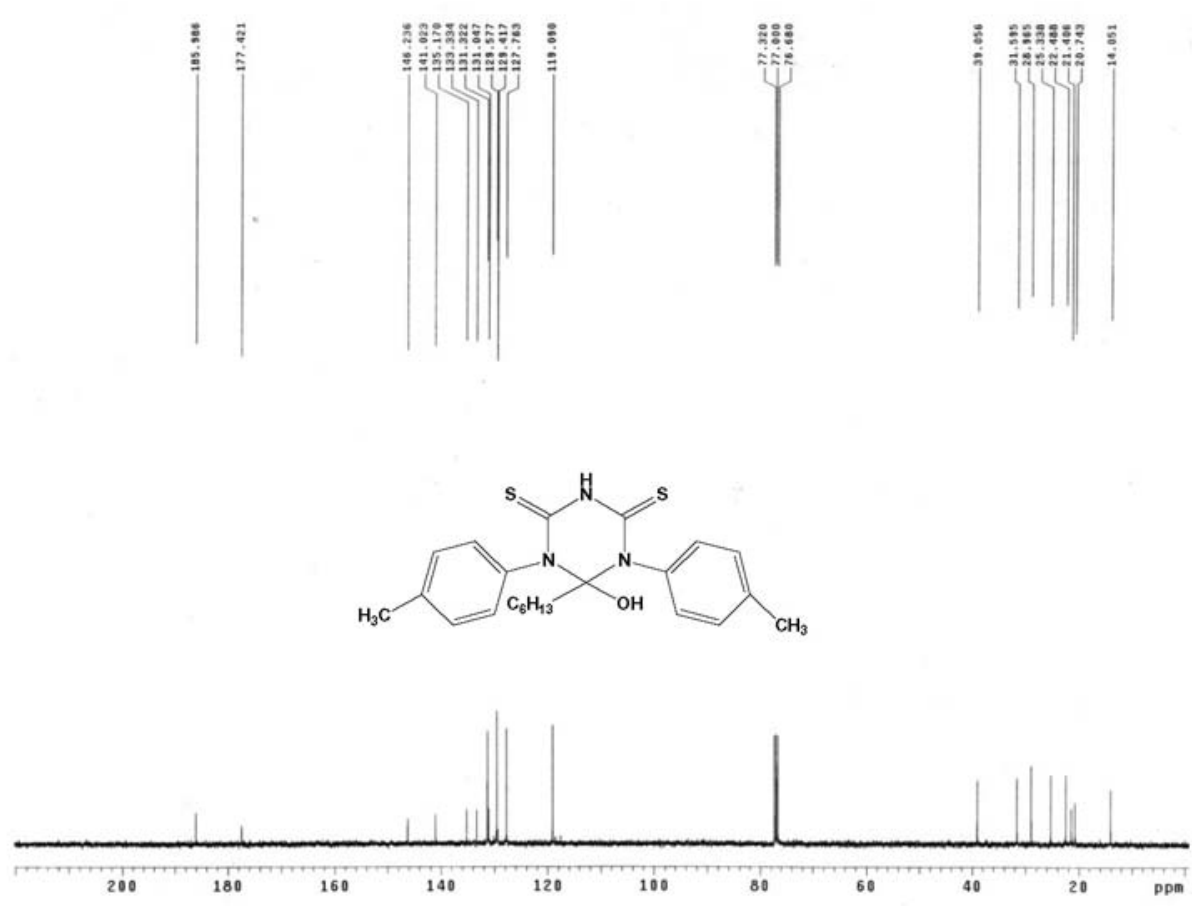

Figure S45. ${ }^{13} \mathrm{C}$ NMR $\left(\mathrm{CDCl}_{3}\right)$ spectrum of 6-hydroxy-6-hexyl-1,5-di(4-methylphenyl)-1,3,5-triazinane-2,4-dithione (15).

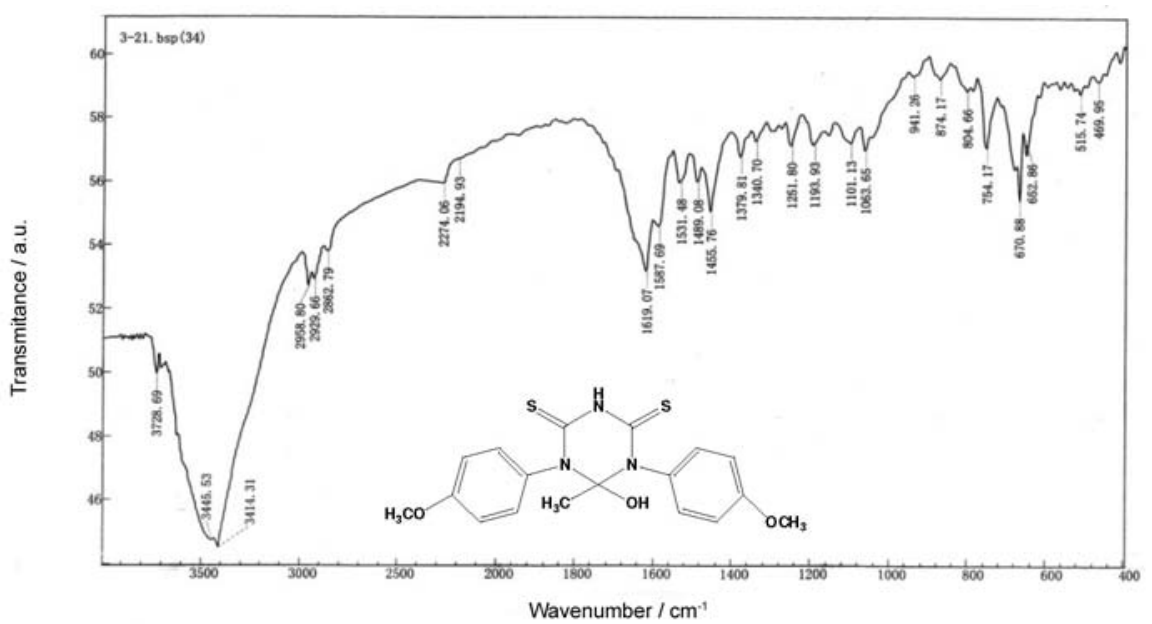

Figure S46. IR (KBr) spectrum of 6-hydroxy-6-methyl-1,5-di(4-methoxyphenyl)-1,3,5-triazinane-2,4-dithione (16). 


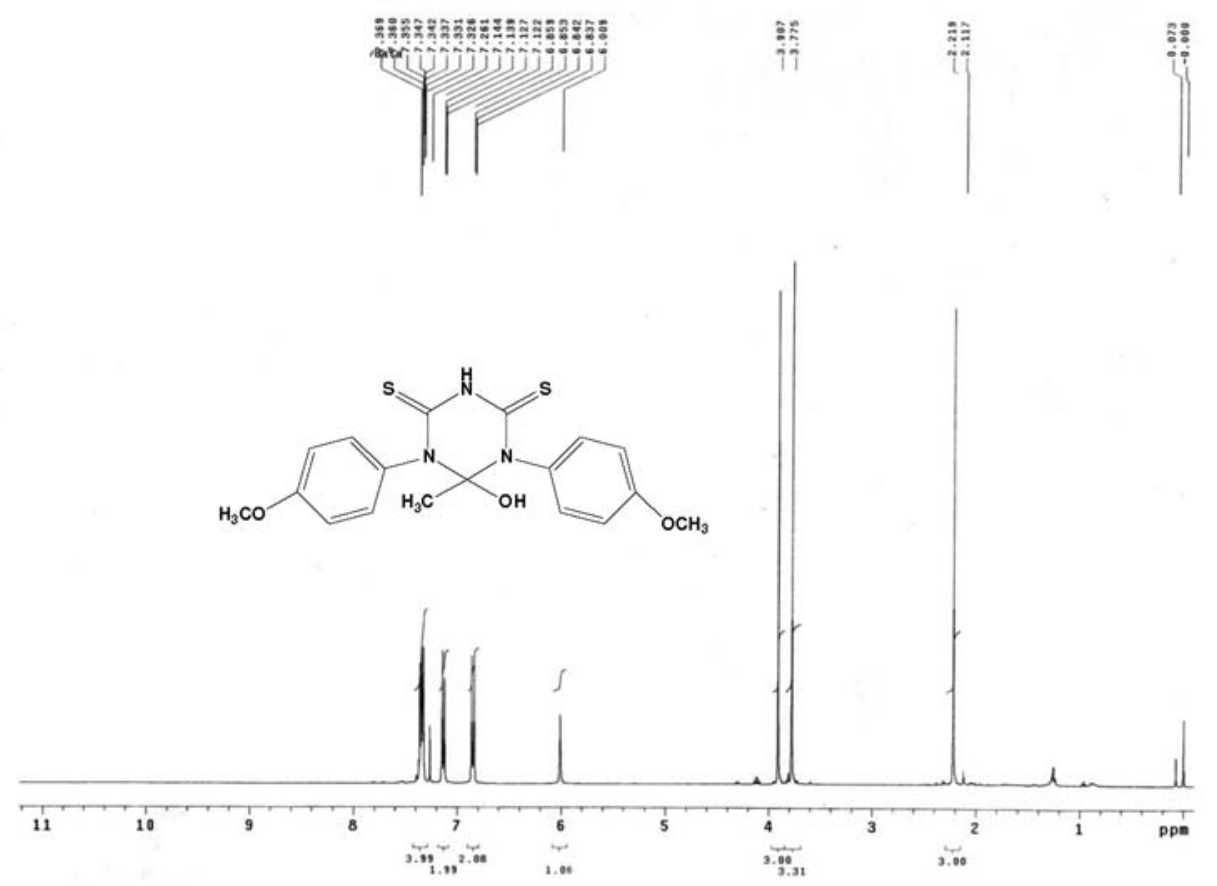

Figure S47. ${ }^{1} \mathrm{H}$ NMR $\left(\mathrm{CDCl}_{3}\right)$ spectrum of 6-hydroxy-6-methyl-1,5-di(4-methoxyphenyl)-1,3,5-triazinane-2,4-dithione (16).
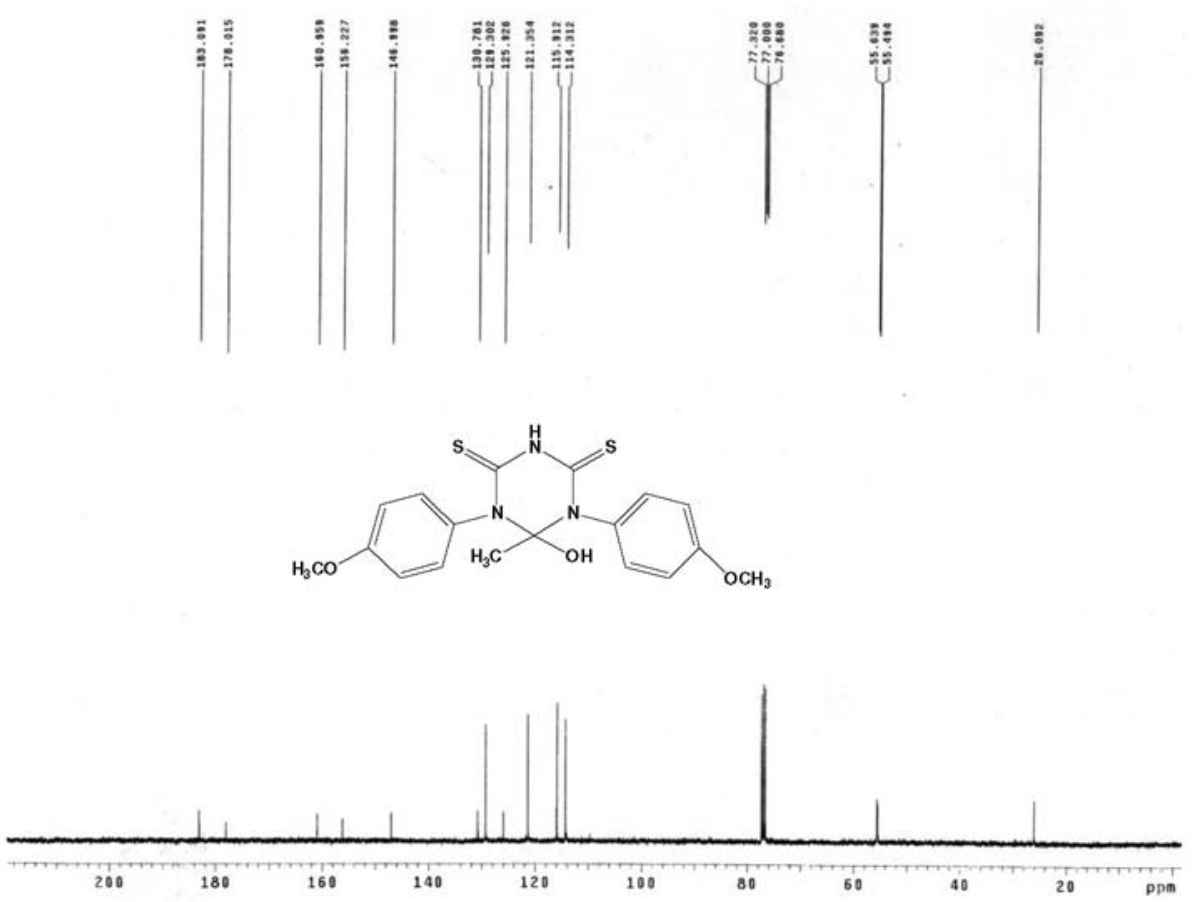

Figure S48. ${ }^{13} \mathrm{C}$ NMR $\left(\mathrm{CDCl}_{3}\right)$ spectrum of 6-hydroxy-6-methyl-1,5-di(4-methoxyphenyl)-1,3,5-triazinane-2,4-dithione (16). 


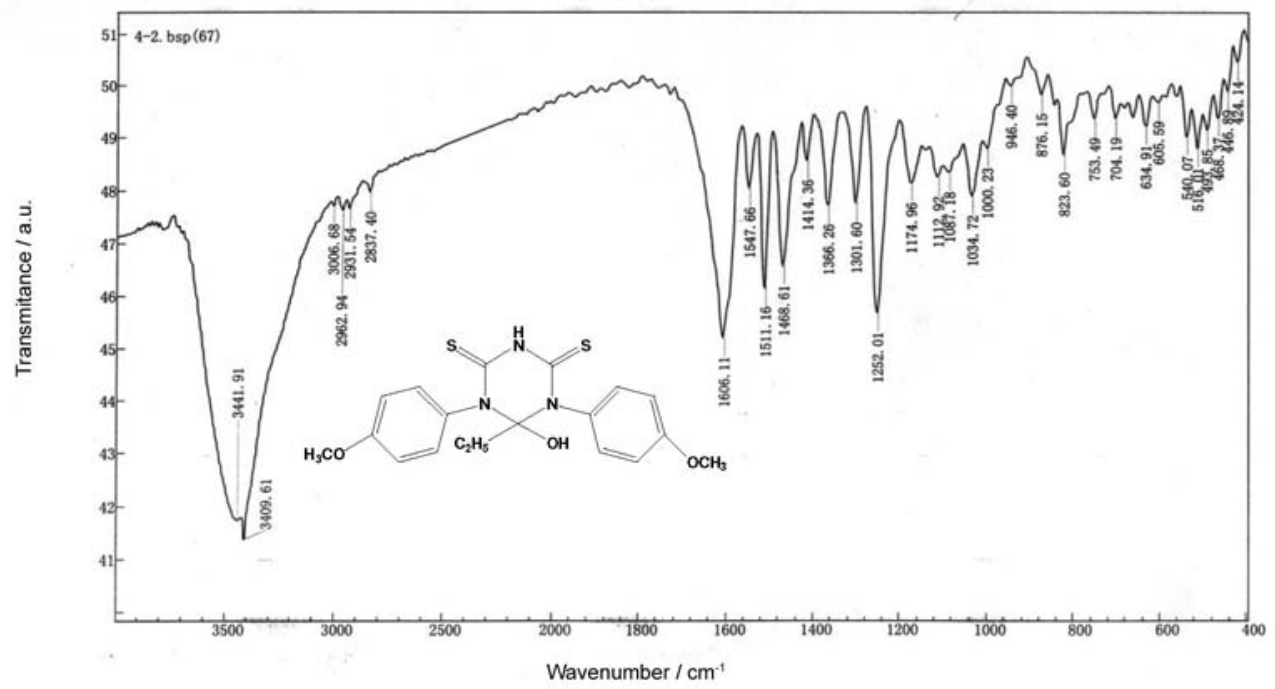

Figure S49. IR (KBr) spectrum of 6-hydroxy-6-ethyl-1,5-di(4-methoxyphenyl)-1,3,5-triazinane-2,4-dithione (17).

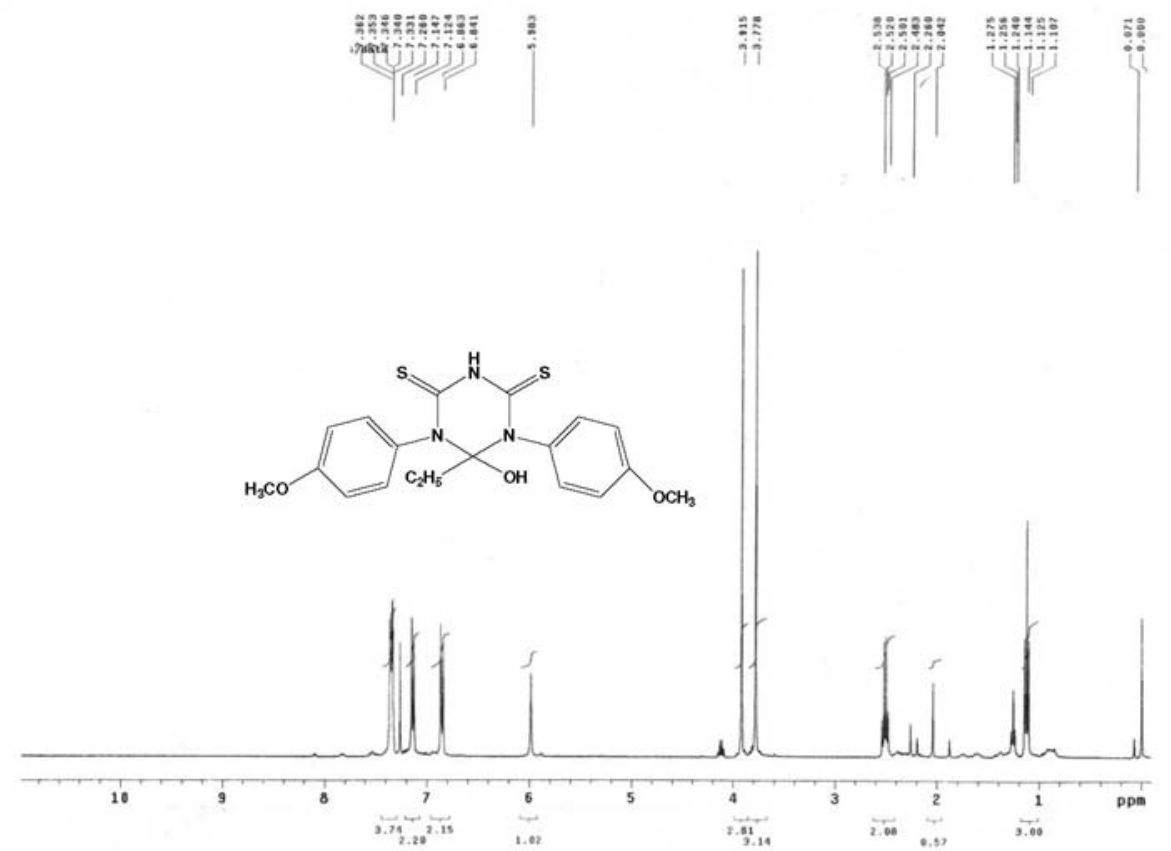

Figure S50. ${ }^{1} \mathrm{H}$ NMR $\left(\mathrm{CDCl}_{3}\right)$ spectrum of 6-hydroxy-6-ethyl-1,5-di(4-methoxyphenyl)-1,3,5-triazinane-2,4-dithione (17). 

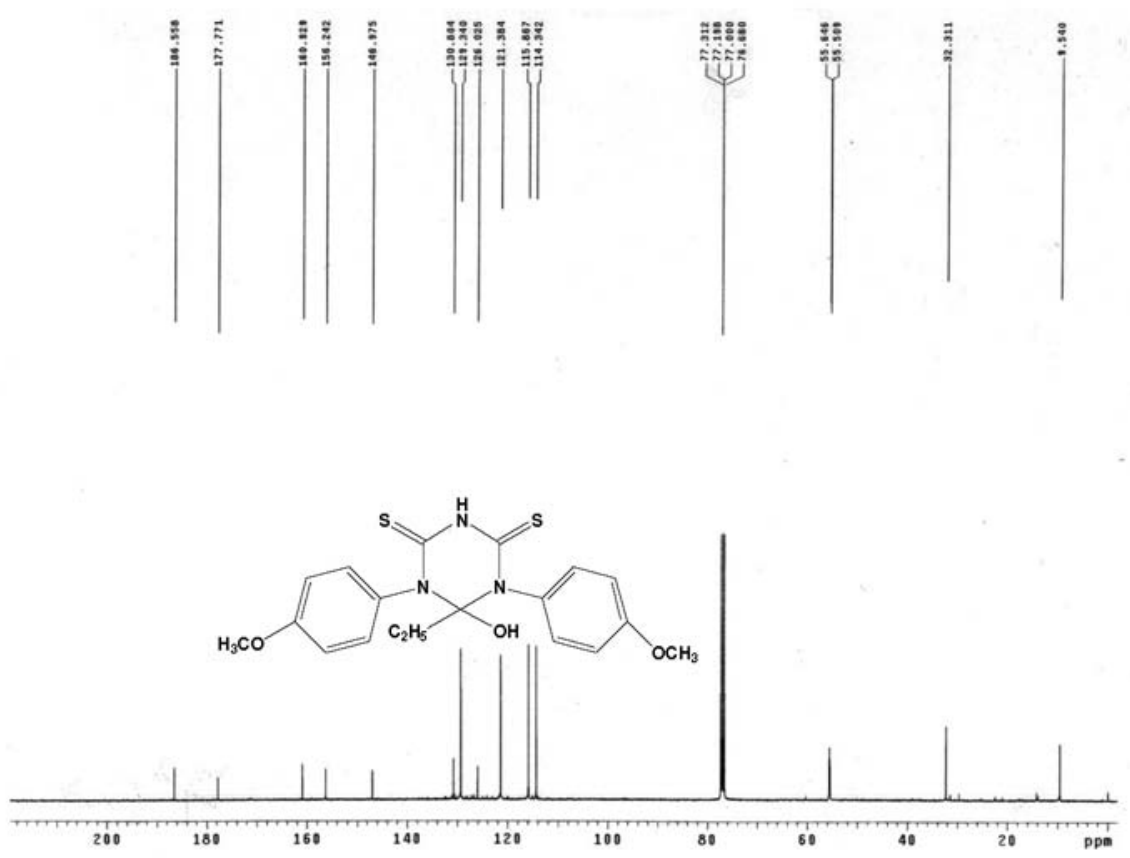

Figure S51. ${ }^{13} \mathrm{C} \mathrm{NMR}\left(\mathrm{CDCl}_{3}\right)$ spectrum of 6-hydroxy-6-ethyl-1,5-di(4-methoxyphenyl)-1,3,5-triazinane-2,4-dithione (17).

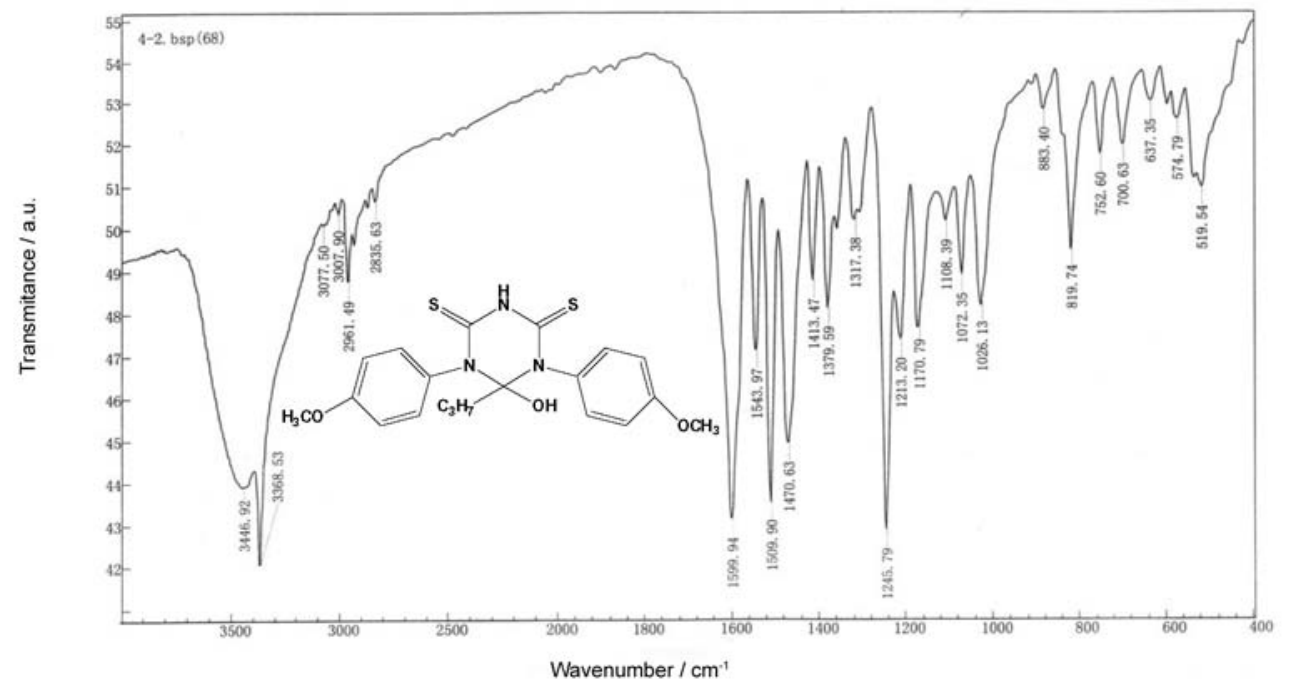

Figure S52. IR (KBr) spectrum of 6-hydroxy-6-propyl-1,5-di(4-methoxyphenyl)-1,3,5-triazinane-2,4-dithione (18). 


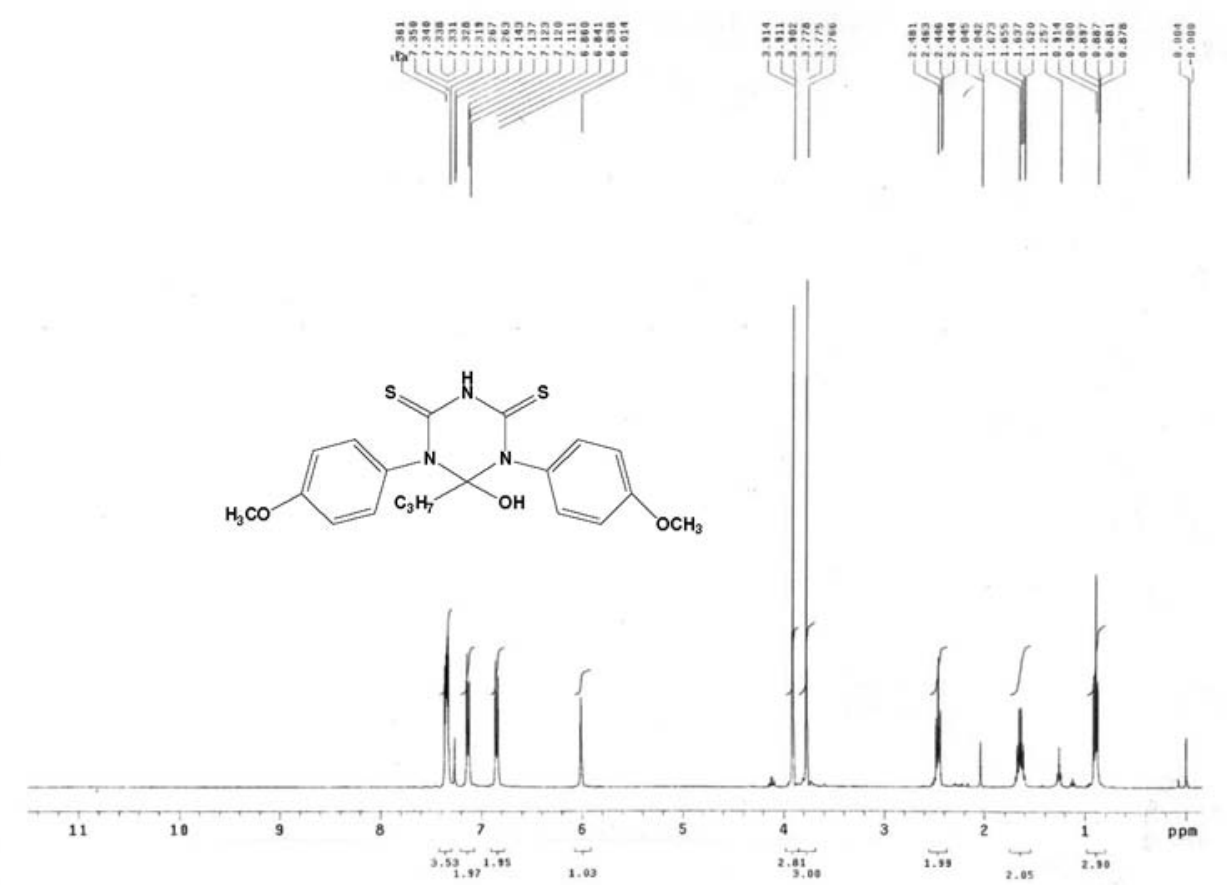

Figure S53. ${ }^{1} \mathrm{H}$ NMR $\left(\mathrm{CDCl}_{3}\right)$ spectrum of 6-hydroxy-6-propyl-1,5-di(4-methoxyphenyl)-1,3,5-triazinane-2,4-dithione (18).
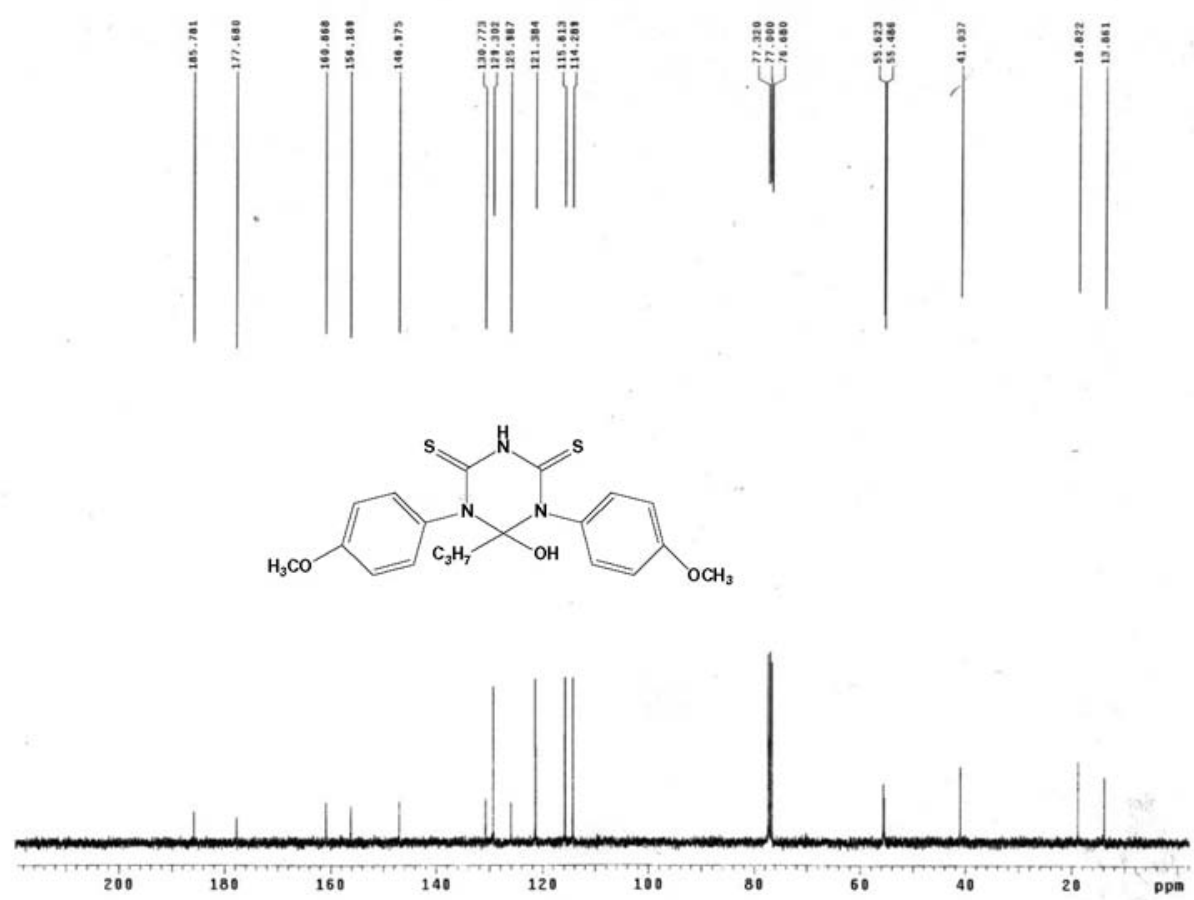

Figure S54. ${ }^{13} \mathrm{C}$ NMR $\left(\mathrm{CDCl}_{3}\right)$ spectrum of 6-hydroxy-6-propyl-1,5-di(4-methoxyphenyl)-1,3,5-triazinane-2,4-dithione (18). 


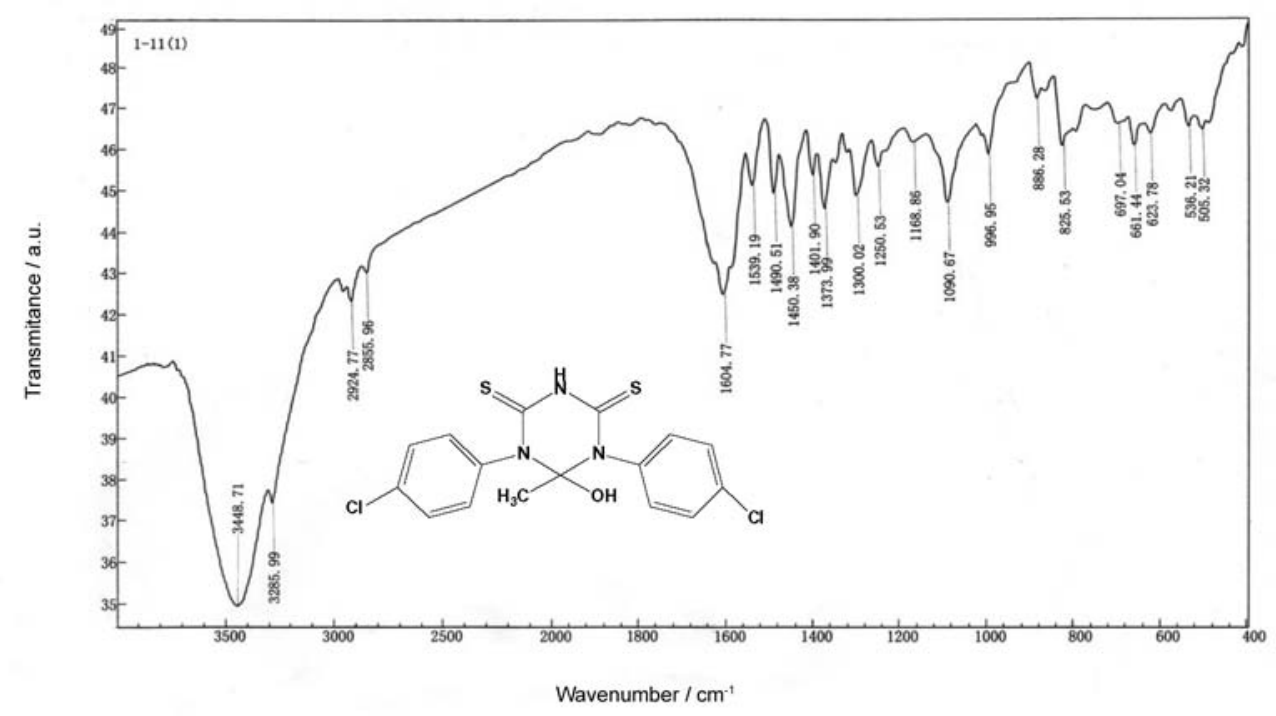

Figure S55. IR (KBr) spectrum of 6-hydroxy-6-methyl-1,5-di(4-chlorophenyl)-1,3,5-triazinane-2,4-dithione (19).

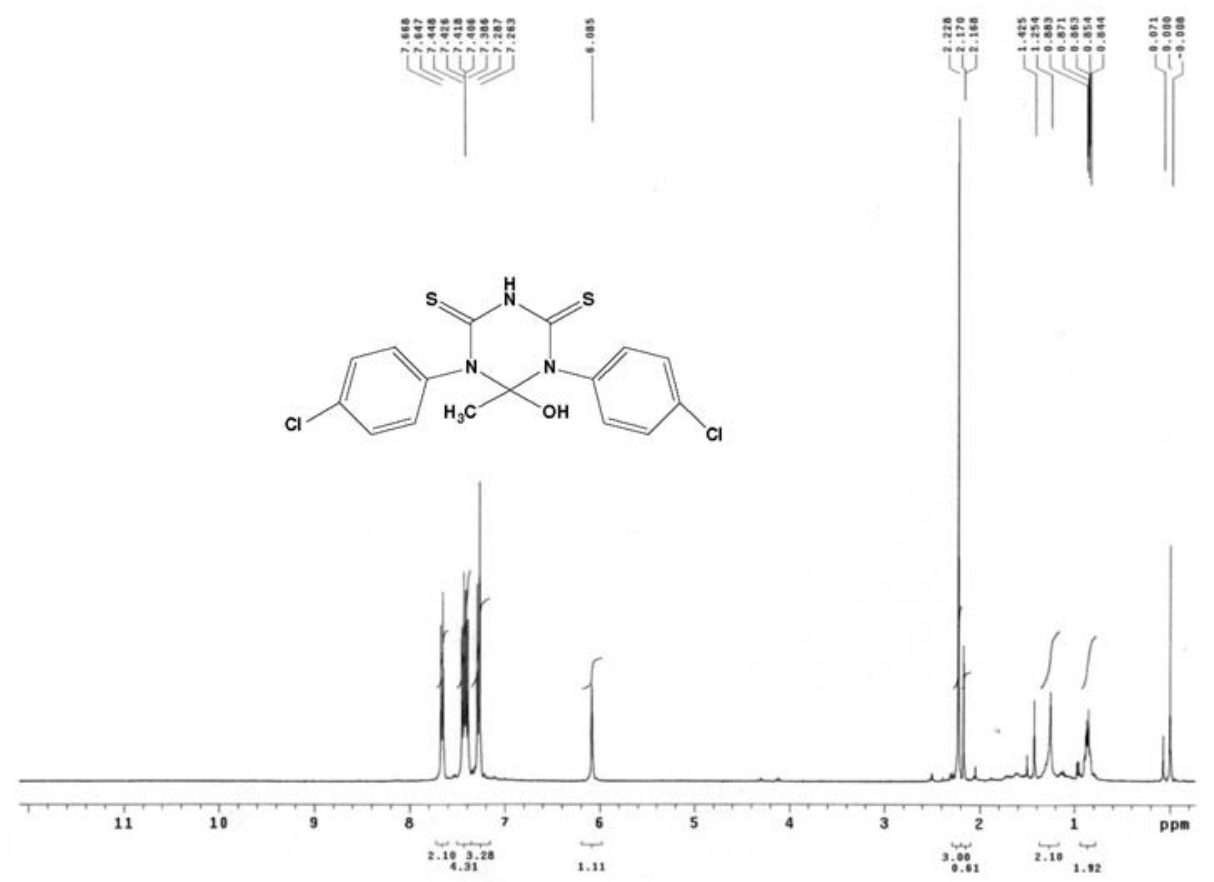

Figure S56. ' $\mathrm{H}$ NMR $\left(\mathrm{CDCl}_{3}\right)$ spectrum of 6-hydroxy-6-methyl-1,5-di(4-chlorophenyl)-1,3,5-triazinane-2,4-dithione (19). 

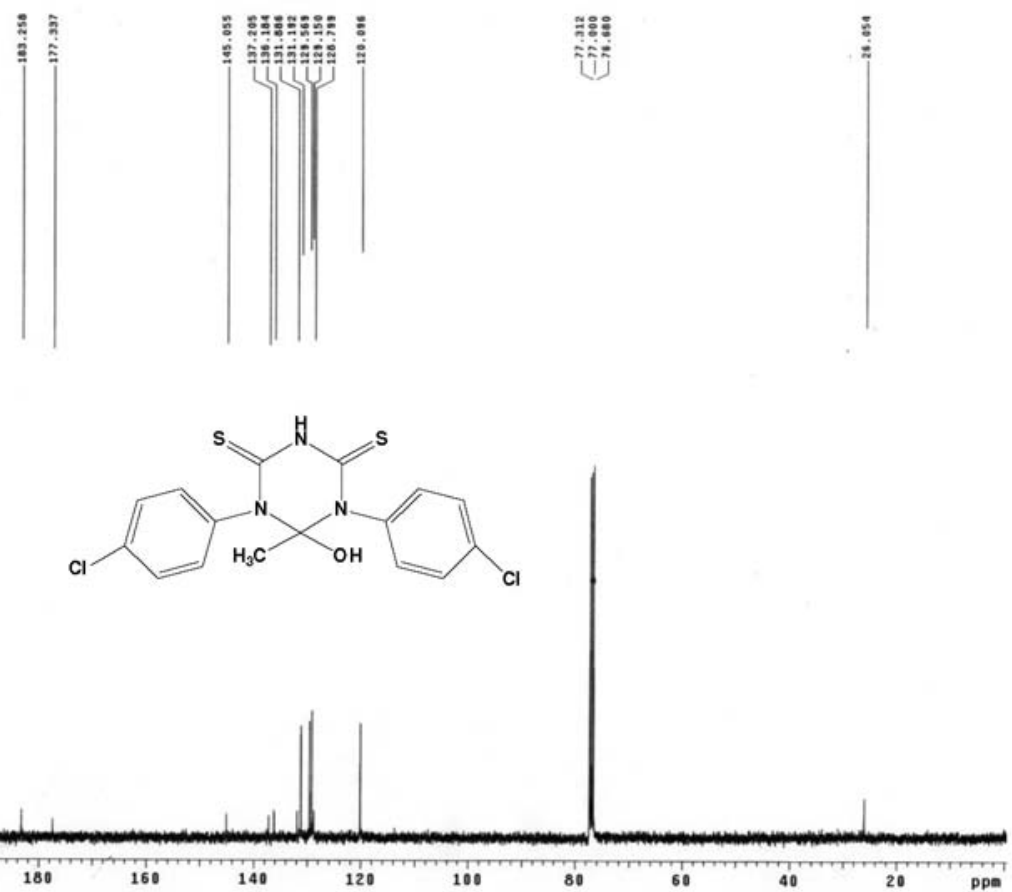

Figure S57. ${ }^{13} \mathrm{C} \mathrm{NMR}\left(\mathrm{CDCl}_{3}\right)$ spectrum of 6- ydroxyl-6-methyl-1,5-di(4-chlorophenyl)-1,3,5-triazinane-2,4-dithione (19). 